CERN-PH-TH/2009-166

DCPT/09/136

IPPP/09/068

SLAC-PUB-13782

arXiv:0909.3240 [hep-ph]

\title{
From the LHC to Future Colliders
}

\author{
CERN Theory Institute Summary Report
}

\author{
A. De Roeck ${ }^{a, b}$, J. Ellis ${ }^{a}$, C. Grojean ${ }^{a, c}$, S. Heinemeyer ${ }^{d}$, \\ K. Jakobs ${ }^{e}$, G. Weiglein ${ }^{f}$, J. Wells ${ }^{a}$ (organizers) \\ G. Azuelos ${ }^{g}$, S. Dawson ${ }^{h}$, B. Gripaios ${ }^{a}$, T. Han $^{i}$, J. Hewett ${ }^{j}$, M. Lancaster ${ }^{k}$, C. Mariotti ${ }^{l}$, \\ F. Moortgat ${ }^{m}$, G. Moortgat-Pick ${ }^{f}$, G. Polesello ${ }^{n}$, S. Riemann ${ }^{p}$, M. Schumacher ${ }^{e}$ (convenors) \\ K. Assamagan ${ }^{h}$, P. Bechtle ${ }^{p}$, M. Carena ${ }^{q}$, G. Chachamis ${ }^{r}$, K.F. Chen ${ }^{s}$, S. De Curtis ${ }^{t}$, K. Desch ${ }^{u}$, \\ M. Dittmar ${ }^{a}$, H. Dreiner ${ }^{v}$, M. Dührssen ${ }^{e}$, B. Foster ${ }^{a u}$, M.T. Frandsen ${ }^{w, x}$, A. Giammanco ${ }^{y}$, \\ R. Godbole ${ }^{z}$, P. Govoni ${ }^{a a}$, J. Gunion ${ }^{a b}$, W. Hollik ${ }^{a c}$, W.S. Hou ${ }^{s}$, G. Isidori ${ }^{a d}$, A. Juste ${ }^{q}$, \\ J. Kalinowski ${ }^{a e}$, A. Korytov ${ }^{a f}$, E. Kou ${ }^{a g}$, S. Kraml ${ }^{a h}$, M. Krawczyk ${ }^{a e}$, A. Martin ${ }^{a i}$, D. Milstead ${ }^{a j}$, \\ V. Morton-Thurtle ${ }^{f}$, K. Moenig ${ }^{o}$, B. Mele ${ }^{a k}$, E. Ozcan $^{k}$, M. Pieri ${ }^{a l}$, T. Plehn ${ }^{a m}$, L. Reina $^{a n}$, \\ E. Richter-Was ${ }^{a, a p}$, T. Rizzo ${ }^{j}$, K. Rolbiecki ${ }^{f}$, F. Sannino ${ }^{w}$, M. Schram ${ }^{a q}$, J. Smillie $^{f}$, \\ S. Sultansoy ${ }^{a r}$, J. Tattersall ${ }^{f}$, P. Uwer ${ }^{a s}$, B. Webber ${ }^{a t}$, P. Wienemann ${ }^{u}$.
}

\begin{abstract}
Discoveries at the LHC will soon set the physics agenda for future colliders. This report of a CERN Theory Institute includes the summaries of Working Groups that reviewed the physics goals and prospects of LHC running with 10 to $300 \mathrm{fb}^{-1}$ of integrated luminosity, of the proposed sLHC luminosity upgrade, of the ILC, of CLIC, of the $\mathrm{LHeC}$ and of a muon collider. The four Working Groups considered possible scenarios for the first $10 \mathrm{fb}^{-1}$ of data at the LHC in which (i) a state with properties that are compatible with a Higgs boson is discovered, (ii) no such state is discovered either because the Higgs properties are such that it is difficult to detect or because no Higgs boson exists, (iii) a missing-energy signal beyond the Standard Model is discovered as in some supersymmetric models, and (iv) some other exotic signature of new physics is discovered. In the contexts of these scenarios, the Working Groups reviewed the capabilities of the future colliders to study in more detail whatever new physics may be discovered by the LHC. Their reports provide the particle physics community with some tools for reviewing the scientific priorities for future colliders after the LHC produces its first harvest of new physics from multi-TeV collisions.
\end{abstract}


${ }^{a}$ CERN, Department of Physics, Geneva, Switzerland

${ }^{b}$ University of Antwerp, Wilrijk, Belgium

${ }^{c} \mathrm{IPhT}$, CEA-Saclay, France

${ }^{d}$ Instituto de Física de Cantabria (CSIC-UC), Santander, Spain

${ }^{e}$ Physikalisches Institut, Albert-Ludwigs-Universität, Freiburg, Germany

${ }^{f}$ IPPP, University of Durham, Durham, UK

${ }^{g}$ Universite de Montréal, Montréal, Canada and TRIUMF, Vancouver, Canada

${ }^{h}$ Physics Department, Brookhaven National Laboratory, Upton New York, USA

${ }^{i}$ Department of Physics, University of Wisconsin, Madison, USA

${ }^{j}$ SLAC National Accelerator Laboratory, Menlo Park, USA

${ }^{k} \mathrm{UCL}$, London, UK

${ }^{l}$ INFN, Sezione di Torino, Italy

${ }^{m}$ Department of Physics, ETH Honggerberg, Zurich, Switzerland

${ }^{n}$ INFN, Sezione di Pavia, Italy

${ }^{\circ}$ DESY, Zeuthen, Germany

${ }^{p}$ DESY, Hamburg, Germany

${ }^{q}$ Fermi National Accelerator Laboratory, Batavia, USA

${ }^{r}$ Paul Scherrer Institut, Villigen, Switzerland

${ }^{s}$ Department of Physics, National Taiwan University, Taipei, Taiwan

${ }^{t}$ Department of Physics, University of Florence and INFN, Sezione di Firenze, Italy

${ }^{u}$ Universität Bonn, Physikalisches Institut, Bonn, Germany

${ }^{v}$ Bethe Center for Theoretical Physics and Physikalisches Institut, Bonn University, Germany

${ }^{w} \mathrm{CP}^{3}$ - Origins, University of Southern Denmark, Odense, Denmark

${ }^{x}$ Rudolf Peierls Centre for Theoretical Physics, University of Oxford, UK

${ }^{y} \mathrm{CP}$, Université Catholique de Louvain, Louvain-la-Neuve, Belgium

${ }^{z}$ Centre for High Energy Physics, Indian Institute of Science, Bangalore, India

${ }^{a}$ Università and INFN Milano-Bicocca

${ }^{a b}$ Department of Physics, UC Davis, USA

${ }^{a c}$ Max-Planck-Institut für Physik (Werner-Heisenberg-Institut), München, Germany

${ }^{a d}$ INFN, Laboratori Nazionali di Frascati, Frascati, Italy

${ }^{a e}$ Physics Department, University of Warsaw, Warsaw, Poland

${ }^{a f}$ University of Florida, Gainesville, USA

${ }^{a g}$ Laboratoire de l'Accelerateur Lineaire, Université Paris-Sud 11, Orsay, France 
${ }^{a h}$ LPSC, UJF Grenoble 1, CNRS/IN2P3, Grenoble, France

${ }^{a i}$ Department of Physics, Sloane Laboratory, Yale University, New Haven, USA

${ }^{a j}$ Fysikum, Stockholms Universitet, Stockholm, Sweden

${ }^{a k}$ INFN, Sezione di Roma, and Università "La Sapienza", Roma, Italy

${ }^{a l}$ University of California San Diego, USA

${ }^{a m}$ Institute for Theoretical Physics, Heidelberg University, Heidelberg, Germany

${ }^{a n}$ Physics Department, Florida State University, Tallahassee, USA

${ }^{a o}$ Institute of Physics, Jagellonian University, Krakow, Poland

${ }^{a p}$ Institute of Nuclear Physics IFJ-PAN, Krakow, Poland

${ }^{a q}$ McGill University, Montréal, Canada

${ }^{a r}$ Physics Division, TOBB University of Economics and Technology, Ankara, Turkey

${ }^{a s}$ Institut für Physik, Humboldt-Universität zu Berlin, Berlin, Germany

${ }^{a t}$ Cavendish Laboratory, J.J. Thomson Avenue, Cambridge, UK

${ }^{a u}$ Particle Physics, University of Oxford, Keble Road, Oxford. UK 


\section{Contents}

Executive summary . . . . . . . . . . . . . . . . . . 2

$1 \quad$ WG1: Higgs ............................ 7

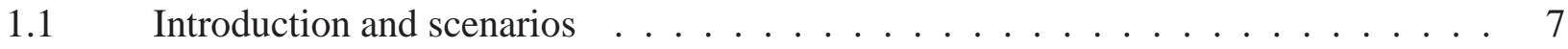

1.2 Observations at the $\mathrm{LHC}_{10 / \text { fb }}$ for a SM-like Higgs boson . . . . . . . . . . 8

1.3 Investigation of the Higgs sector at $\mathrm{LHC}_{10 / \mathrm{fb}} \ldots \ldots \ldots \ldots$

1.4 From the $\mathrm{LHC}_{10 / \mathrm{fb}}$ to future colliders . . . . . . . . . . . . . . 13

1.5 Summary and conclusions of $\mathrm{WG1} \ldots \ldots \ldots$

2 WG2: No Higgs boson . . . . . . . . . . . . . . . . . . 22

$2.1 \quad$ Introduction . . . . . . . . . . . . . . . . . 22

2.2 Unobserved Higgs boson scenarios . . . . . . . . . . . . . . . . . . . 23

2.3 Higgsless/technicolor scenarios . . . . . . . . . . . . . . . . 31

$2.4 \quad$ Summary of $\mathrm{WG} 2 \ldots \ldots \ldots \ldots \ldots \ldots$

3 WG3: Missing Energy . . . . . . . . . . . . . . . . . . 43

3.1 Introduction and scenarios . . . . . . . . . . . . . 43

3.2 Commissioning of Missing Transverse Energy in the ATLAS and CMS experiments 43

$3.3 \quad$ Discovery at $\mathrm{LHC} \ldots \ldots \ldots \ldots$. . . . . . . . . . . . . . . . . . 48

$3.4 \quad$ Measurement at $\mathrm{LHC} \ldots \ldots \ldots \ldots \ldots$

3.5 The LHC-Dark Matter Connection . . . . . . . . . . . . . . . . . . 54

3.6 Future Lepton Colliders . . . . . . . . . . . . . . . . . 55

3.7 Summary and conclusions of WG3 . . . . . . . . . . . . . . 62

$4 \quad$ WG4: Other new physics signatures . . . . . . . . . . . . . . . . . . 64

$4.1 \quad$ Introduction and scenarios . . . . . . . . . . . . . . . . . . 64

$4.2 \quad Z^{\prime}$ production at future colliders . . . . . . . . . . . . . 65

$4.3 \quad 4$ th Generation of Fermions . . . . . . . . . . . . . . . . . . . 68

4.4 Testing Seesaw mechanisms at the LHC . . . . . . . . . . . . . . . . 71

$4.5 \quad$ New signatures and implications for detectors on new colliders . . . . . . . . . 74

4.6 Black Holes . . . . . . . . . . . . . . . . . . . . 76

$4.7 \quad$ Flavour Physics . . . . . . . . . . . . . . . . . . . . . . . . 79

4.8 Summary and conclusions of WG4 . . . . . . . . . . . . . . 81

Appendix: Future Colliders Overview Series . . . . . . . . . . . . . . . . . . . 83

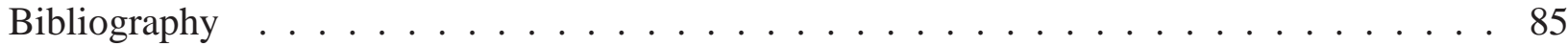




\section{Executive summary}

The LHC is about to initiate the direct exploration of physics at the TeV scale. Ground-breaking discoveries may be possible with the first few inverse femtobarns of data, which would certainly have profound implications for the future of the field of particle physics and beyond. The results obtained at the LHC will set the agenda for the future colliders that will be required to study any new physics in more detail. Once early LHC data have been analysed, the world-wide particle physics community will need to converge on a strategy for shaping the future of particle physics. Given the fact that the complexity and size of possible future accelerator experiments will require a long construction time, the decision of when and how to go ahead with a future major facility will have to be taken in a timely fashion. Several projects for future colliders are being developed, and in a few years time it will be necessary to set priorities between these options, informed by whatever new physics the LHC may reveal.

This CERN Theory Institute brought together theorists, experimentalists and machine physicists from around the world to discuss - before the actual start of data taking at the LHC - the physics goals, capabilities and possible results of the LHC, and how these relate to future possible collider programmes. The plan of the Theory Institute was (i) to discuss recent physics developments, (ii) to anticipate the near-term capabilities of the Tevatron, LHC and other experiments, and (iii) to discuss the most effective ways to be prepared to provide scientific input to plans for the future direction of the field. The following points were addressed in particular: physics progress and results prior to LHC collisions, initial goals and prospects for the 2010 LHC physics run, and the subsequent physics goals and prospects in the early LHC phase with $10 \mathrm{fb}^{-1}$ (we refer to this

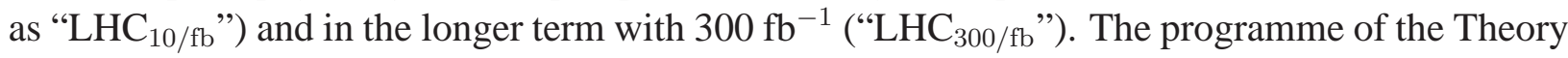
Institute was structured according to the questions: 1) What have we learned from data accrued up to this point, and what may we expect to know from new physics during the initial phase of LHC operation? 2) What do we need to know from the LHC for planning future accelerators? 3) What scientific strategies will be needed to advance from the planned LHC running to a future collider facility?

To answer the last two questions, participants studied in particular what can be expected from the LHC with a specific early luminosity, namely $10 \mathrm{fb}^{-1}\left(\mathrm{LHC}_{10 / \mathrm{fb}}\right)$, in different scenarios for $\mathrm{TeV}$-scale physics, and which strategy for future colliders one would adopt in each case. In order to address these questions, the Theory Institute efforts were organized into four broad categories of possible signatures in the early LHC data: (i) a Higgs candidate (and anything else), (ii) no Higgs candidate (and anything else), (iii) missing energy, (iv) more exotic signals of new physics. Four Working Groups studied details of each of these different scenarios.

Key considerations for the Working Groups were the scientific benefits of various future upgrades of the LHC compared with the feasibility and timing of possible future colliders. For this reason, the programme also included a series of talks overviewing future colliders, one on each possible accelerator followed by a talk on the specific physics interest of that collider, including the Tevatron, the (s)LHC, the ILC, the LHeC, CLIC and a muon collider.

Working Group 1 assessed the implications of the possible detection of a state with properties that are compatible with a Higgs boson, whether Standard Model (SM)-like or not. If Nature has chosen a SM-like Higgs, then ATLAS and CMS are well placed to discover it with $10 \mathrm{fb}^{-1}$ and measure its mass. However, measuring its other characteristics (decay width, spin, CP properties, 
branching ratios, couplings, ...) with an accuracy better than 20 to $30 \%$, and correlating them with precision top studies and electroweak precision physics, will require some future collider.

In the Higgs mass region below $\sim 130 \mathrm{GeV}$ the LHC experiments will probe various production modes (gluon and weak-boson fusion) and decay modes $(\gamma \gamma, \tau \tau$ and eventually $\bar{b} b$ final states), and a 5- $\sigma$ discovery in the early phase with $10 \mathrm{fb}^{-1}$ will probably require combining these channels. In that context, the Tevatron will add valuable complementary information, in particular via the Higgs search in the important $W / Z H, H \rightarrow \bar{b} b$ channels. On the other hand, the LHC with $300 \mathrm{fb}^{-1}$ could measure, with mild theory assumptions, the couplings of a low-mass Higgs boson to some fermions with an accuracy of 15 to $30 \%$, and the Higgs couplings to gauge bosons to about $10 \%$, but will provide no access to Higgs self-couplings. The sLHC could increase the accuracy of the measurements of the Higgs couplings and may give some access to the Higgs self-couplings in the mass region around $160 \mathrm{GeV}$ (though this requires further simulation), and would also be sensitive to rare decays of a light Higgs boson. The ILC would allow precise measurements of all the quantum numbers and many couplings of the Higgs boson, in addition to its mass and width, yielding in this way a nearly complete profile of the Higgs boson. If the Higgs boson is relatively light, CLIC could give access to more of its rare decay modes of the Higgs boson and improve access to the Higgs self-couplings, and produce any resonances weighing up to $2.5 \mathrm{TeV}$ in $W W$ scattering.

Working Group 2 considered scenarios in which no state is detected with the first $10 \mathrm{fb}^{-1}$ of LHC data with properties that are compatible with a Higgs boson. It reviewed complementary physics channels such as gauge boson self-couplings, longitudinal vector-boson scattering, exotic Higgs scenarios, and scenarios with invisible Higgs decays. If no clear Higgs-like signal has been established with the first $10 \mathrm{fb}^{-1}$ of LHC data, one needs to consider two generic classes of scenarios: those in which a Higgs exists but is difficult to see, and those in which no Higgs exists at all.

Three specific examples of the former scenarios were studied: models with complex parameters such as the supersymmetric CPX scenario, models with unexpected visible Higgs decays, and models with invisible Higgs decays. Also studied were four "Higgsless" scenarios: walking technicolour models, scenarios with extra dimensions, models in which extra dimensions are deconstructed (i.e., replaced by a set of discrete points) and models with strong $W W$ scattering.

In many of these scenarios, with higher LHC luminosity (e.g., with the sLHC) it should be possible to determine whether or not a Higgs boson exists, e.g., by improving the sensitivity to the production and decays of Higgs-like particles or vector resonances, or by measuring $W W$ scattering. The ILC would enable precision measurements of even the most difficult-to-see Higgs bosons, as would CLIC. The latter would be also good for producing heavy resonances. Which future collider option is to be preferred may well depend on other early LHC physics results, e.g., whether the LHC discovers other new physics such as supersymmetry or extra dimensions, or whether there is other evidence from the LHC or elsewhere for CP-violating effects beyond the SM. In particular, if other new physics is detected that seems to hint at the realisation of (at least one) fundamental Higgs state in nature, e.g., supersymmetric particles are produced and / or the gauge sector does not show indications of strong electroweak symmetry breaking dynamics, then this could be a strong case for an $e^{+} e^{-}$linear collider to explore the expected mass range for the Higgs and to determine precisely the nature of the other observed new physics.

In considering missing-energy signatures at the LHC, Working Group 3 used supersymmetry 
as a representative model. The signals studied included events with leptons and jets, with a view to measuring the masses, spins and quantum numbers of any new particles produced.

Studies of the LHC capabilities at $\sqrt{s}=14 \mathrm{TeV}$ show that $100 \mathrm{pb}^{-1}$ of luminosity would enable the overall energy scale of the missing-energy physics to be determined with an accuracy of $8 \%$, if the mass scale of supersymmetry (or other missing-energy physics) is near the lower limit of the range still allowed by lower-energy experiments. With $1 \mathrm{fb}^{-1}$ of LHC luminosity, signals of missing energy with one or more additional leptons would give sensitivity to a large range of supersymmetric mass scales. Several ways to measure the masses of individual sparticle masses were discussed, aimed at dealing with the difficulties presented by the missing energymomentum vector. This also creates difficulties for spin measurements, which would benefit from more information about the reference frame of the decaying particle.

In all the missing-energy scenarios studied, early LHC data would provide important input for the technical and theoretical requirements for future linear collider physics, such as the detector capabilities (e.g., resolving mass degeneracies could require exceptionally good jet energy resolution), running scenarios, required threshold scans and upgrade options (e.g., for a $\gamma \gamma$ collider and/or GigaZ).

In many scenarios, the missing energy is carried away by dark-matter particles, generating a very important connection with cosmology. Characterizing the nature of the missing-energy scenario, e.g., so as to be able to use data to calculate the dark-matter density, will (for many supersymmetric scenarios) be difficult with LHC data alone, and a future linear collider would help greatly in this analysis.

Working Group 4 studied examples of phenomena that do not involve a missing-energy signature, such as the production of a new $Z^{\prime}$ boson, other leptonic resonances, a fourth generation of fermions and exotic quarks, lepton-number-violating signals, the impact of new physics on observables in the flavour sector, TeV-scale gravity signatures, heavy stable charged particles and other exotic signatures of new physics. In general, determining the properties of such new physics phenomena, for instance by measuring couplings and angular distributions, will require a much larger number of events and more precise measurements than what is needed for the discovery of such phenomena.

The sLHC luminosity upgrade has the capability to add crucial information on the properties of any new physics discovered during early LHC running, as well as increasing the search sensitivity. On the other hand, a future linear collider, with its clean environment, known initial state and polarised beams, is unparalleled in terms of its abilities to conduct ultra-precise measurements of new and SM phenomena, as long as the new physics scale is within reach of the machine. For example, in the case of a $Z^{\prime}$, high-precision measurements at a future linear collider would provide a mass reach that is about ten times higher than the centre-of-mass energy of the linear collider itself.

Generally speaking, the physics capabilities of the sLHC, the ILC and CLIC are relatively well understood, but will need refinement in light of initial LHC running. In cases where the exploration of new physics might be challenging at the early LHC, synergy with a linear collider could be very beneficial. In particular a staged approach to linear-collider energies seems very promising from the physics point of view, and should be further investigated. On the other hand, the physics cases for the $\mathrm{LHeC}$ or a muon collider have yet to be established. The prospects of the $\mathrm{LHeC}$ for enlarging the coverage of the LHC will depend crucially on the specific scenario 
of $\mathrm{TeV}$-sale physics realised in Nature. In the case of the muon collider, a background-saturated environment, the challenge of making vertex measurements and the lack of polarised beams, as well as the significant loss of forward coverage due to shielding, will make precision measurements more challenging than at a linear collider. The exploration of some new physics phenomena may ultimately call for very high energies. For instance, in a scenario where the fundamental Planck scale is in the TeV range, a complete mapping of the energy regime five to ten times above the Planck scale would require an energy upgrade of the LHC (DLHC) or even a Very Large Hadron Collider (VLHC).

As already emphasized, the physics cases for all these projects will need to be reviewed after data from the initial LHC running are analyzed. Some physics scenarios envisage new physics at a relatively low energy scale, such as a light Higgs boson or some low-mass supersymmetric particles. On the other hand, some scenarios such as those with high-mass vector resonances or strong $W W$ scattering suggest that new physics may appear only at high energies. Even in scenarios with new low-mass physics, their full exploration may also require higher energies, e.g., to measure the properties of strongly-interacting sparticles or higher-lying Kaluza-Klein excitations in scenarios with extra dimensions.

The purpose of this Theory Institute was not to arbitrate between these scenarios and the corresponding priorities for future colliders, but rather to provide the particle physics community with some tools for such considerations when the appropriate time comes. Novel results from the early LHC data may open the way towards an exciting future for particle physics made possible by a new major facility. In order to seize this opportunity, the particle physics community will need to agree on convincing and scientifically solid motivations for such a facility. The intention of this Theory Institute was to provide a framework for discussing now how this could be achieved, before actual LHC results start to come in. We now look forward to the first multi-TeV collisions in the $\mathrm{LHC}$, and to the harvest of new physics they will provide. 


\section{Acknowledgements}

The CERN TH Institute has been funded by the CERN TH unit and by 'MassTeV' ERC advanced grant 226371. This work has been supported in part by the European Community's Marie-Curie Research Training Network under contract MRTN-CT-2006-035505 'Tools and Precision Calculations for Physics Discoveries at Colliders' (HEPTOOLS) and MRTN-CT-2006-035657 'Understanding the Electroweak Symmetry Breaking and the Origin of Mass using the First Data of ATLAS' (ARTEMIS). This work was supported in part by the DOE under Task TeV of contract DE-FGO2-96-ER40956. J.H. and T.R. were supported by the U.S. Department of Energy under

Contract DE-AC02-76SF00515. M.L. was supported by the Science and Technology Facilities Council, UK. 


\section{WG1: Higgs}

S. Dawson, S. Heinemeyer, C. Mariotti, M. Schumacher (convenors)

K. Assamagan, P. Bechtle, M. Carena, G. Chachamis, K. Desch, M. Dittmar, H. Dreiner, M. Dührssen, R. Godbole, S. Gopalakrishna, W. Hollik, A. Juste, A. Korytov, S. Kraml, M. Krawczyk, K. Moenig, B. Mele, M. Pieri, T. Plehn, L. Reina, E. Richter-Was, P. Uwer, G. Weiglein

The prospects for a Higgs boson discovery with $10 \mathrm{fb}^{-1}$ at the LHC are summarized and the implications of such a discovery for future colliders such as the sLHC, the ILC, and CLIC are discussed in this section.

\subsection{Introduction and scenarios}

Identifying the mechanism of electroweak symmetry breaking will be one of the main goals of the LHC and other future high-energy physics experiments. Many possibilities have been studied in the literature, of which the most popular ones are the Higgs mechanism in the Standard Model (SM) and the Minimal Supersymmetric Standard Model (MSSM). Assuming that a new state which is a possible candidate for a Higgs boson has been observed, the full identification of the mechanism of electroweak symmetry breaking will require the measurement of all its characteristics. This comprises an accurate mass determination, a (model-independent) measurement of its individual couplings to other particles (i.e. not only the ratio of couplings), a determination of the self-couplings to confirm the "shape" of the Higgs potential, as well as measurements of its spin and $\mathcal{C P}$-quantum numbers, etc. These measurements will most probably only be partially possible at the LHC, even running at high luminosity. It will be up to future colliders to complete the Higgs profile.

We first review what we might know about the Higgs sector once the LHC has collected $10 \mathrm{fb}^{-1}$ at a center-of-mass energy of $14 \mathrm{TeV}$ (called $\mathrm{LHC}_{10 / \mathrm{fb}}$ in the following) and has observed an object compatible with a Higgs boson. Secondly, we investigate the capabilities of future colliders to further unravel the mechanism responsible for electroweak symmetry breaking and to confirm that a Higgs boson has indeed been observed. The discussion of the second step will be split into three scenarios:

- A: Observation of a SM-like Higgs boson with a mass $130 \mathrm{GeV} \lesssim M_{H} \lesssim 180 \mathrm{GeV}$. This mass range theoretically allows the SM to be valid until the Planck scale. SM-like means that no statistically significant deviations of the properties of the Higgs boson from the expectations of the $\mathrm{SM}$ can be observed at the $\mathrm{LHC}_{10 / \mathrm{fb}}$. It should be kept in mind that a SM-like Higgs boson in the mass range of $160 \mathrm{GeV} \leq M_{H}^{\mathrm{SM}} \leq 170 \mathrm{GeV}$ has recently been excluded at the $95 \%$ C.L. by the Tevatron [1].

- B: Observation of a SM-like Higgs boson outside the above mass range of $130 \mathrm{GeV}$ to $180 \mathrm{GeV}$.

- C: Observation of a non-SM-like Higgs boson (e.g. signal rates or coupling structures deviate from SM expectations). See Section 2.2 for a discussion of various scenarios with unusual couplings. 
Scenario C, or Scenario B with $M_{H} \gtrsim 180 \mathrm{GeV}$, typically imply additional signs of new physics besides a single Higgs boson.

\subsection{Observations at the $\mathrm{LHC}_{10 / \mathrm{fb}}$ for a SM-like Higgs boson}

Most quantitative analyses at ATLAS and CMS have been performed for a SM-like Higgs boson. Consequently, we summarize and comment on the potential of $\mathrm{LHC}_{10 / \mathrm{fb}}$ to observe a Higgs boson assuming SM-like couplings.

We will not try to disentangle and explain differences in the discovery potentials between ATLAS and CMS. Details can be found in the original publications of the ATLAS [2] and CMS [3] collaborations, which contain information on how the discovery potentials have been evaluated. However, we will briefly mention existing differences between the experimental results that might be relevant for the subsequent discussion.

\begin{tabular}{|l||c|c|c|c|c|}
\hline channel $/ M_{H}[\mathrm{GeV}]$ & 110 & 115 & 120 & 125 & 130 \\
\hline \hline ATLAS $H \rightarrow \gamma \gamma$ cuts & - & 2.0 & 2.4 & - & 2.7 \\
\hline ATLAS $H \rightarrow \gamma \gamma$ opt. & - & - & 3.6 & - & 4.3 \\
\hline ATLAS $q q \rightarrow q q H, H \rightarrow \tau \tau$ & 2.4 & - & 2.9 & - & 2.5 \\
\hline ATLAS $H \rightarrow W W \rightarrow e \mu \nu \nu+0$ Jets & - & - & - & - & 3.4 \\
\hline ATLAS $H \rightarrow W W \rightarrow e \mu \nu \nu+2$ Jets & - & - & - & - & 2.0 \\
\hline ATLAS $H \rightarrow Z Z \rightarrow 4$ leptons & - & - & 1.5 & - & 3.5 \\
\hline CMS $H \rightarrow \gamma \gamma$ cuts & - & 3.1 & 3.3 & - & 3.5 \\
\hline CMS $H \rightarrow \gamma \gamma$ opt. & - & 5.3 & 5.7 & - & 4.7 \\
\hline CMS $q q \rightarrow q q H, H \rightarrow \tau \tau \rightarrow l$ had & - & 2.2 & - & 2.0 & - \\
\hline CMS $H \rightarrow W W \rightarrow l l \nu \nu$ & - & - & 0.4 & - & 0.9 \\
\hline CMS $H \rightarrow Z Z \rightarrow 4$ leptons & - & 2.6 & 2.3 & - & 5.3 \\
\hline
\end{tabular}

Table 1.1: Summary of the significances for observation of a SM-like Higgs boson in various search channels for masses below $130 \mathrm{GeV}$ in the ATLAS and CMS experiments after collecting $10 \mathrm{fb}^{-1}$.

The discovery potentials for a SM-like Higgs boson in three different mass ranges of the Higgs boson using the ATLAS and CMS detectors are shown in Tables 1.1 - 1.3. As mentioned above, some differences between ATLAS and CMS can be observed. One difference can be seen in the channel $p p \rightarrow H \rightarrow \gamma \gamma$, where the CMS results look more optimistic, especially in the "optimized" analysis [3]. ATLAS has also performed an optimized analysis for the $H \rightarrow \gamma \gamma$ decay mode and it is expected that the sensitivity can be increased by $50 \%$ relative to the results shown in Table 1.1. The $H \rightarrow W^{+} W^{-}$decay mode looks more promising in the ATLAS analysis than in the CMS studies. In the ATLAS study, only the final state with one electron and one muon has been analyzed. Taking into account also the di-electron and di-muon final states, it is expected that the significance of an observation will increase by a factor of up to about $\sqrt{2}$. For the $H \rightarrow$ $\tau^{+} \tau^{-}$decay mode, ATLAS has investigated the $\tau^{+} \tau^{-} \rightarrow l^{+} l^{-} X$ and $\tau^{+} \tau^{-} \rightarrow l^{ \pm} \mathrm{had}^{\mp} X$ final 


\begin{tabular}{|l||c|c|c|c|c|c|c|}
\hline channel $/ M_{H}[\mathrm{GeV}]$ & 135 & 140 & 145 & 150 & 160 & 170 & 180 \\
\hline \hline ATLAS $H \rightarrow \gamma \gamma$ cuts & - & 2.2 & - & - & - & - & - \\
\hline ATLAS $H \rightarrow \gamma \gamma$ opt. & - & 4.0 & - & - & - & - & - \\
\hline ATLAS $q q \rightarrow q q H, H \rightarrow \tau \tau$ & - & 1.9 & - & - & - & - & - \\
\hline ATLAS $H \rightarrow W W \rightarrow e \mu \nu \nu+0$ Jets & - & 5.8 & - & 8.4 & 10.6 & 10.2 & 7.1 \\
\hline ATLAS $H \rightarrow W W \rightarrow e \mu \nu \nu+2$ Jets & - & 3.0 & - & 4.1 & 5.1 & 5.1 & 4.2 \\
\hline ATLAS $H \rightarrow Z Z \rightarrow 4$ leptons & - & 6.3 & - & 7.3 & 4.1 & - & 2.9 \\
\hline CMS $H \rightarrow \gamma \gamma$ cuts & - & 3.2 & - & 2.3 & - & - & - \\
\hline CMS $H \rightarrow \gamma \gamma$ opt. & - & 3.9 & - & - & - & - & - \\
\hline CMS $q q \rightarrow q q H, H \rightarrow \tau \tau \rightarrow l$ had & 2.1 & - & 0.8 & - & - & - & - \\
\hline CMS $H \rightarrow W W \rightarrow l l \nu \nu$ & - & 1.3 & - & 2.9 & 6.3 & 6.3 & 4.8 \\
\hline CMS $H \rightarrow Z Z \rightarrow 4$ leptons & - & 7.8 & - & 9.0 & 5.4 & 2.6 & 4.5 \\
\hline
\end{tabular}

Table 1.2: Summary of the significances for observation of a SM-like Higgs boson in various search channels for $130<M_{H} \leq 180 \mathrm{GeV}$ in the ATLAS and CMS experiment after collecting $10 \mathrm{fb}^{-1}$.

\begin{tabular}{|l||c|c|c|c|c|c|c|c|c|c|}
\hline channel $/ M_{H}[\mathrm{GeV}]$ & 190 & 200 & 250 & 300 & 350 & 400 & 450 & 500 & 550 & 600 \\
\hline \hline ATLAS $H \rightarrow Z Z \rightarrow 4$ leptons & - & 8.3 & - & 7.2 & - & 6.0 & - & 2.9 & - & 1.8 \\
\hline CMS $H \rightarrow W W \rightarrow l l \nu \nu$ & 2.2 & 1.3 & - & - & - & - & - & - & - & - \\
\hline CMS $H \rightarrow Z Z \rightarrow 4$ leptons & 9.1 & 9.2 & 7.7 & 8.0 & 8.1 & 7.8 & 6.6 & 5.2 & 4.1 & 3.2 \\
\hline
\end{tabular}

Table 1.3: Summary of the significances for observation of a SM-like Higgs boson in various search channels for masses above $180 \mathrm{GeV}$ in the ATLAS and CMS experiment after collecting $10 \mathrm{fb}^{-1}$.

states, whereas CMS has only considered the latter one $\left(\tau \tau \rightarrow l^{ \pm} \mathrm{had}^{\mp}\right.$ refers to one $\tau$ decaying leptonically and the other one hadronically).

Most analyses up to now have been performed for $30 \mathrm{fb}^{-1}$. In order to arrive at the data shown in Tables $1.1-1.3$ the following rescaling and extrapolation methods were applied: The ATLAS numbers are taken from Ref. [2] and have been obtained by taking the square root of the $-2 \ln \lambda$ values quoted there (where $\lambda$ denotes the profile likehood ratio of the background over the signal plus background hypothesis). The CMS numbers are based on Ref. [3], where numbers for $30 \mathrm{fb}^{-1}$ are reported. The numbers above are obtained by scaling the number of signal and background events by a factor of $1 / 3$, but using the relative uncertainties from the original analysis (which might be optimistic).

Both experiments currently do not consider the associated production with a pair of topquarks and subsequent decay to a pair of b-quarks $(t \bar{t} H, H \rightarrow b \bar{b})$ as a discovery mode for initial data taking. The latest sensitivity studies quote statistical significances at a mass of $M_{H}=$ $120 \mathrm{GeV}$ corresponding to 1.8 to 2.2 with $30 \mathrm{fb}^{-1}$ in the ATLAS experiment [2] using the semileptonic decay mode only. In the CMS experiment, combining all possible final states a significance of 1.6 to 2.4 with $60 \mathrm{fb}^{-1}$ is reached [3]. Including current estimates of systematic background 

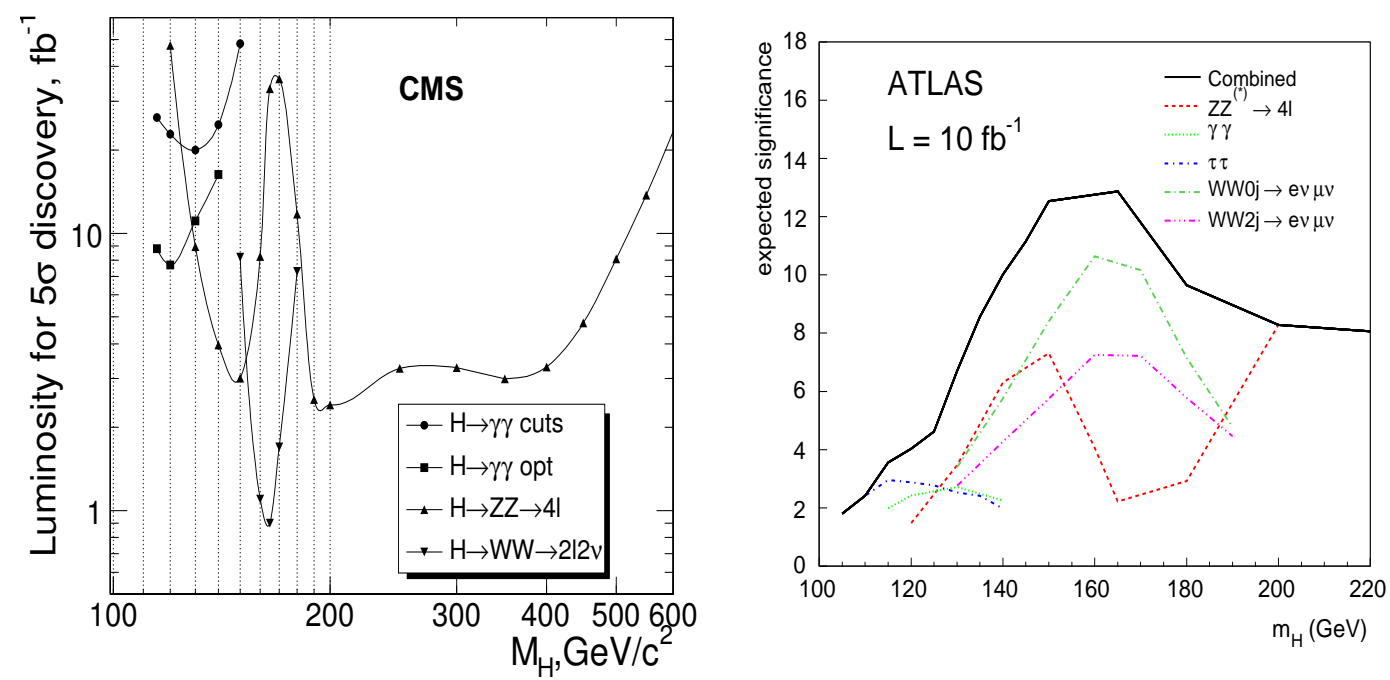

Fig. 1.1: Left: Luminosity needed for a $5 \sigma$ discovery at CMS [3]. Right: Expected significance at ATLAS with $10 \mathrm{fb}^{-1}[2]$.

uncertainties, the significance is below 0.5 for the integrated luminosities assumed [2,3]. According to recent NLO calculations of the background [4,5], yielding a relatively large $K$-factor of $\sim 1.8$, the prospects for this channel are even more doubtful. Alternatives to possibly recover some sensitivity for the $H \rightarrow b \bar{b}$ channel are discussed in the next section.

In Fig. 1.1, the expected performances of the ATLAS and CMS detectors are shown as a function of $M_{H}$ (assuming SM rates). CMS shows the luminosity needed for a $5 \sigma$ discovery, while ATLAS shows the expected significance after $10 \mathrm{fb}^{-1}$.

\section{$\mathrm{LHC}$ at $\sqrt{s}=10 \mathrm{TeV}$}

The LHC is expected to initially run at $\sqrt{s}=10 \mathrm{TeV}$. At this energy, production rates are typically reduced by about a factor of two from those at $\sqrt{s}=14 \mathrm{TeV}$. Both CMS and ATLAS have performed preliminary studies of the expected Higgs sensitivities at $\sqrt{s}=10 \mathrm{TeV}$ [6,7], see also [8]. In the combined $H \rightarrow Z Z+W^{+} W^{-}$channel, CMS estimates that the required luminosity for a 95\% C.L. exclusion limit is roughly doubled at $\sqrt{s}=10 \mathrm{TeV}$ for $M_{H}$ between 120 and $200 \mathrm{GeV}$ from that at $\sqrt{s}=14 \mathrm{TeV}$. In the mass range $M_{H}=160-170 \mathrm{GeV}$, a 95\% C.L. exclusion limit is obtained in this channel with $\sim 0.2 \mathrm{fb}^{-1}$ (as compared with $0.1 \mathrm{fb}^{-1}$ at $\sqrt{s}=14 \mathrm{TeV}$ ). Similarly, ATLAS has examined the combined $H \rightarrow W^{+} W^{-} \rightarrow 2 l$ with 0 and 2 jets channel and finds that a $5 \sigma$ discovery is possible with $\sim 1 \mathrm{fb}^{-1}$ for $M_{H} \sim 160-170 \mathrm{GeV}$ at $\sqrt{s}=10 \mathrm{TeV}$.

The preliminary findings above are partially obtained using a fast simulation of the detectors, without optimisation of the selections for running at $10 \mathrm{TeV}$ and using the relative systematic uncertainties from earlier studies assuming sometimes larger integrated luminosities. Hence the results have not the same level of maturity as those in Refs. [2,3], but yield an indicative estimate of the sensitivity during early data taking. 


\section{Higgs searches at the Tevatron}

By the time the ATLAS and CMS collaborations have analyzed $10 \mathrm{fb}^{-1}$, the Tevatron Run II will have been completed [9]. If the Tevatron runs in 2011, a total of $10 \mathrm{fb}^{-1}$ analyzed per experiment (CDF and $\mathrm{D} \varnothing)$ is expected. The latest projections by the Tevatron experiments [10] suggest that with this luminosity, a 95\% C.L. exclusion of the SM Higgs in the mass range 114-185 GeV could be achieved (where $114.4 \mathrm{GeV}$ is the limit obtained for a SM-like Higgs boson at LEP [11, 12]). In addition, a $3 \sigma$ sensitivity is expected for $M_{H}<115 \mathrm{GeV}$ and $150 \mathrm{GeV}<M_{H}<180 \mathrm{GeV}$. This means that the significance of a SM Higgs signal would be $\sim 2-3 \sigma$ for $M_{H}<150 \mathrm{GeV}$. While this is the overall sensitivity from the combination of all search channels, in particular, for $M_{H}<130 \mathrm{GeV}$, most of the sensitivity comes from $V H(V=W, Z)$, with $H \rightarrow b \bar{b}$. This is complementary with the LHC, which in this mass range mainly probes $H \rightarrow \gamma \gamma$ and $H \rightarrow$ $\tau^{+} \tau^{-}$, demonstrating the complementarity between both machines. Therefore, the Tevatron could potentially yield interesting information on $\sigma\left(p \bar{p} \rightarrow V^{*} \rightarrow V H\right) \times \mathrm{BR}(H \rightarrow b \bar{b})$ for $M_{H}<$ $130 \mathrm{GeV}$, which could be used in a global analysis of Higgs couplings at the LHC, (see below).

\subsection{Investigation of the Higgs sector at $\mathrm{LHC}_{10 / \mathrm{fb}}$}

After the observation of a new resonance at the $\mathrm{LHC}_{10 / \mathrm{fb}}$ the first goal will be to measure its characteristics (mass, width, branching ratios, couplings, ... ). Only if the profile agrees completely (within sufficiently small experimental errors) with that predicted for a SM Higgs boson, one could be convinced that the SM Higgs mechanism is realized in nature.

The accuracy of the determination of a Higgs boson mass will crucially depend on the decay modes observable. Assuming SM properties, the precision is dominated by the decay $H \rightarrow \gamma \gamma$ at low masses and by $H \rightarrow Z Z^{(*)}$ at higher masses. From the $H \rightarrow \gamma \gamma$ channel a precision better than $\sim 1 \%$ can be expected at the $\mathrm{LHC}_{10 / \mathrm{fb}}$ (rescaling the numbers from Ref. [3]). For higher masses CMS has shown that a statistical error of $\lesssim 0.4 \%$ on the mass measurement can be reached assuming $30 \mathrm{fb}^{-1}$ in the $H \rightarrow Z Z^{*} \rightarrow 4$ lepton channel for Higgs boson masses below $180 \mathrm{GeV}$ [3]. Even for a Higgs boson mass of $600 \mathrm{GeV}$, the expected precision is $2.4 \%$.

In order to verify that the resonance observed is indeed a Higgs boson, it will be crucial to measure its couplings to all particle species. A study was performed in 2004 assuming at least an integrated luminosity of $2 \times 30 \mathrm{fb}^{-1}$ [13] ("combining" ATLAS and CMS). This analysis, however, used now outdated results from ATLAS and CMS. The analysis assumed SM production and decay rates. Another assumption employed was that the coupling to SM gauge bosons is

bounded from above by $g_{H V V}^{2}<\left(g_{H V V}^{\mathrm{SM}}\right)^{2} \times 1.05$ (which is realized in all models with Higgs singlets and doublets only, including the MSSM). For Higgs boson masses below $150 \mathrm{GeV}$ the results depend strongly on the observability of the $H \rightarrow b \bar{b}$ decay mode since it dominates the total decay width. The corresponding results for the $\mathrm{LHC}_{10 / \mathrm{fb}}$ are obtained from Ref. [13] by rescaling. Table 1.4 summarizes estimated precisions on the absolute couplings as well as the total and invisible (or undetectable) Higgs width. It has been found that new negative contributions to the $g g H$ and $\gamma \gamma H$ (loop induced) couplings could be detected at the $-50 \%$ level. However, it should be kept in mind that these analyses assume a measurement of the $t \bar{t} H, H \rightarrow b \bar{b}$ and $H \rightarrow W W^{(*)}$ channel and are thus to be taken with care.

Given the new findings for the $t \bar{t} H, H \rightarrow b \bar{b}$ channel $[2,3]$, the decay to $b \bar{b}$ will hardly be 
observable, thus missing a large contribution to the total width, and consequently no coupling determination seems to be possible for Higgs boson masses below $150 \mathrm{GeV}$ at the $\mathrm{LHC}_{10 / \mathrm{fb}}$. New methods to recover the observability of $H \rightarrow b \bar{b}$ need to be studied experimentally in order to regain at least some sensitivity in the low mass region. Several methods have been suggested; e.g., $W H, H \rightarrow b \bar{b}$ with a large boost of the Higgs bosons [14], or Higgs production in vector boson fusion in association with either a central photon in $p p \rightarrow q q H \gamma \rightarrow q q b \bar{b} \gamma$, where the requirement of an extra high- $p_{T}$ photon in the $q q H \rightarrow q q b \bar{b}$ final state dramatically enhances the $S / B$ ratio [15], or an additional $W$ boson in $p p \rightarrow q q H W \rightarrow q q b \bar{b} \ell \nu$, with the final high- $p_{T}$ lepton improving the trigger efficiency $[16,17]$. These strategies are currently being investigated by the ATLAS and CMS collaborations. An updated study of Higgs coupling measurements has been presented in Ref. [18] for $M_{H}=120 \mathrm{GeV}$ (based on the parton-level study in Ref. [14] to recover the decay $H \rightarrow b \bar{b}$ ) with the conclusion that coupling constant measurements with accuracies in the $20-40 \%$ region should be possible with $30 \mathrm{fb}^{-1}$.

Without assumptions about the Higgs model (for instance an upper bound on $g_{H V V}$, see above), one would be left with measurements of ratios of Higgs boson decay widths. The accessible ratios directly correspond to the visible production and decay channels at a given value of $M_{H}$. A rough summary of the estimated precision on the ratios is given in Table 1.5. It is found from Ref. [19] by rescaling to lower luminosity at the $\mathrm{LHC}_{10 / \mathrm{fb}}$.

\begin{tabular}{|l||c|c|c|c|c|c|c|c|}
\hline channel $/ M_{H}[\mathrm{GeV}]$ & 120 & 130 & 140 & 150 & 160 & 170 & 180 & 190 \\
\hline \hline$g_{H W W}$ & $29 \%$ & $25 \%$ & $20 \%$ & $14 \%$ & $9 \%$ & $8 \%$ & $8 \%$ & $9 \%$ \\
\hline$g_{H Z Z}$ & $30 \%$ & $27 \%$ & $21 \%$ & $16 \%$ & $15 \%$ & $19 \%$ & $14 \%$ & $11 \%$ \\
\hline$g_{H \tau \tau}$ & $63 \%$ & $39 \%$ & $38 \%$ & $50 \%$ & & & & \\
\hline$g_{H b b}$ & $72 \%$ & $54 \%$ & $56 \%$ & $73 \%$ & & & & \\
\hline$g_{H t t}$ & $87 \%$ & $62 \%$ & $45 \%$ & $36 \%$ & $31 \%$ & $32 \%$ & $36 \%$ & $45 \%$ \\
\hline \hline$\Gamma_{H}$ & & $77 \%$ & $60 \%$ & $42 \%$ & $27 \%$ & $25 \%$ & $26 \%$ & $29 \%$ \\
\hline$\Gamma_{\text {inv }} / \Gamma_{H}$ & $81 \%$ & $72 \%$ & $56 \%$ & $39 \%$ & $23 \%$ & $20 \%$ & $22 \%$ & $24 \%$ \\
\hline
\end{tabular}

Table 1.4: Summary of the precisions at the $\mathrm{LHC}_{10 / \mathrm{fb}}$, assuming $g_{H W W}^{2}<\left(g_{H W W}^{\mathrm{SM}}\right)^{2} \times 1.05[13,19]$. Upper part: $\delta g_{H x x} / g_{H x x}$; lower part: $\Gamma_{H}$ is the total Higgs width, $\Gamma_{\text {inv }} / \Gamma_{H}$ denotes the sensitivity to an invisible or undetectable width with respect to the total width. "Precisions" larger than $100 \%$ are omitted.

\begin{tabular}{|l||c|c|c|c|c|c|c|c|}
\hline channel $/ M_{H}[\mathrm{GeV}]$ & 120 & 130 & 140 & 150 & 160 & 170 & 180 & 190 \\
\hline \hline$\Gamma_{H Z Z} / \Gamma_{H W W}$ & & $55 \%$ & $36 \%$ & $32 \%$ & $47 \%$ & $78 \%$ & $46 \%$ & $27 \%$ \\
\hline$\Gamma_{H \tau \tau} / \Gamma_{H W W}$ & & $58 \%$ & $62 \%$ & $85 \%$ & & & & \\
\hline$\Gamma_{H \gamma \gamma} / \Gamma_{H W W}$ & $79 \%$ & $53 \%$ & $53 \%$ & $68 \%$ & & & & \\
\hline
\end{tabular}

Table 1.5: Summary of the estimated precision, $\delta\left(\Gamma(H \rightarrow X X) / \Gamma\left(H \rightarrow W W^{(*)}\right)\right) /(\Gamma(H \rightarrow$ $\left.X X) / \Gamma\left(H \rightarrow W W^{(*)}\right)\right)$ on ratios of couplings at the $\mathrm{LHC}_{10 / \mathrm{fb}}$ (see text) [19]. "Precisions" larger than $100 \%$ are omitted. 
The Higgs tri-linear self-coupling, $g_{H H H}$, is a key parameter in the Higgs sector since it describes the "form" of the Higgs potential. The measurement of $g_{H H H}$ allows a stringent test of the SM potential and some discrimination between different models (2HDM, MSSM, baryogenesis, Higgs-Radion mixing, ... ) where the coupling may be significantly enhanced. Unfortunately, at the LHC, even with $\mathcal{L}=300 \mathrm{fb}^{-1}$, no measurement of a SM-like Higgs self-coupling can be expected.

Another measurement that can be made at the $\mathrm{LHC}_{10 / \mathrm{fb}}$ concerns the structure of the tensor coupling of the putative Higgs resonance to weak gauge bosons. This can be studied at $\mathrm{LHC}_{10 / \mathrm{fb}}$ with good precision for some values of $M_{H}$. A study exploiting the difference in the azimuthal angles of the two tagging jets in weak vector boson fusion has shown that for $M_{H}=160 \mathrm{GeV}$ the decay mode into a pair of $W$-bosons (which is maximal at $M_{H}=160 \mathrm{GeV}$ ) allows the discrimination between the SM tensor structure and purely anomalous $\mathcal{C P}$-even and -odd coupling structures at a level of 4.5 to $5.3 \sigma$ assuming the production rate is that of the SM [20-22]. A discriminating power of two standard deviations for the distinction of the $\mathcal{C P}$-even and -odd tensor structure at a mass of $120 \mathrm{GeV}$ in the tau lepton decay mode requires an integrated luminosity of $30 \mathrm{fb}^{-1}$.

\subsection{From the $\mathrm{LHC}_{10 / \mathrm{fb}}$ to future colliders}

The LHC running at high(er) luminosity (subsequently called $\mathrm{LHC}_{300 / \mathrm{fb}}$, assuming the collection of $\sim 300 \mathrm{fb}^{-1}$ per detector) will follow the $\mathrm{LHC}_{10 / \mathrm{fb}}$, expanding the knowledge about the Higgs sector. In this section we will analyze what can be gained from future colliders in the various scenarios beyond what is anticipated from the $\mathrm{LHC}_{300 / \mathrm{fb}}$. As future colliders, we consider the sLHC [23], the ILC [24] and CLIC [25].

Other options could be an LHC with double energy (DLHC), see Ref. [26] and references therein, and a VLHC (Very Large Hadron Collider), with an energy of $\sqrt{s}=40-200 \mathrm{TeV}$ [26,27]. More information can also be found in Refs. [28,29]. The physics case for a DLHC or VLHC will only emerge after discoveries at the LHC, e.g. concerning the potential measurement of the Higgs tri-linear coupling, $g_{H H H}$. Another option could be a $\mu^{+} \mu^{-}$collider [30,31], with an energy of $\sqrt{s} \sim M_{H}$. At a $\mu^{+} \mu^{-}$collider, with an integrated luminosity of $\mathcal{L}^{\text {int }} \lesssim 10 \mathrm{pb}^{-1}$ an ultra-precise measurement of a Higgs boson mass and width would be possible [32] and coupling measurements up to the same level as at the ILC could be performed. The $\mu^{+} \mu^{-}$collider could thus help to determine the Higgs profile. In the following, however, we will not discuss the physics capabilities of a DLHC, VLHC or a $\mu^{+} \mu^{-}$collider, as the technical feasibility studies are in very preliminary stages.

We start by briefly summarizing the existing analyses in the Higgs sector for the $\mathrm{LHC}_{300 / \mathrm{fb}}$, sLHC, ILC and CLIC.

$\mathrm{LHC}_{300 / \mathrm{fb}}$ :

Going to the $\mathrm{LHC}_{300 / \mathrm{fb}}$ will allow the observation of a Higgs boson candidate in more production and decay modes compared to the $\mathrm{LHC}_{10 / \mathrm{fb}}$. This will yield a better determination of ratios of partial widths as well as absolute couplings, provided the $H \rightarrow b \bar{b}$ channel is accessible and assuming that the coupling to weak gauge bosons $g_{H V V}$ is bounded from above by $g_{H V V}^{2}<\left(g_{H V V}^{\mathrm{SM}}\right)^{2} \times 1.05$ [13]. In this study, for $M_{H} \lesssim 150 \mathrm{GeV}$, couplings to fermions could be determined between $\sim 13 \%$ and $\sim 30 \%$, whereas Higgs couplings to gauge bosons could be measured to $10-15 \%(5-10 \%)$ for $M_{H} \lesssim(\gtrsim) 150 \mathrm{GeV}$ (see also Ref. [18]). 
Several studies for the measurement of the tri-linear Higgs coupling, $g_{H H H}$, have been performed, assuming $M_{H} \gtrsim 140 \mathrm{GeV}$ with $H \rightarrow W W^{(*)}$ as the dominant decay mode $[23,33,34]$. The studies conclude that at the $\mathrm{LHC}_{300 / \mathrm{fb}}$ a determination of $g_{H H H}$ will not be possible.

With a larger data sample the spin and $\mathcal{C P}$ quantum numbers can be inferred from the angular distributions of the leptons in the $H \rightarrow Z Z \rightarrow 4 \ell$ decay mode (see Refs. [35-37] and references therein for theory studies). The CMS collaboration considered the case that the observed scalar boson $\phi$ is a mixture of a $\mathcal{C P}$-even $(H)$ and $\mathcal{C P}$-odd $(A)$ boson according to $\Phi=H+\eta A$. Assuming the SM production rate the parameter $\zeta=\arctan \eta$ can be determined to $10-20 \%$ for $M_{H}=$ $200-400 \mathrm{GeV}$ with an integrated luminosity $\mathcal{L}^{\text {int }}=60 \mathrm{fb}^{-1}$ [3]. Using the same observables the ATLAS collaboration found that the hypothesis of non-SM $\mathcal{C P}$ and spin combinations can be distinguished from the SM value at the $95 \%$ C.L. for $M_{H} \gtrsim 250 \mathrm{GeV}$ and $\mathcal{L}^{\text {int }}=100 \mathrm{fb}^{-1}$ [38].

SLHC:

The sLHC is a luminosity upgrade of the LHC which aims for an ultimate luminosity of $1000 \mathrm{fb}^{-1} /$ year sometime after 2018. Assuming that the detector capabilities remain roughly the same as those anticipated for the LHC, the SLHC [23] will increase the discovery potential for high mass objects by $25-40 \%$.

By the time the sLHC is realized, the Higgs discovery phase at the LHC will be largely completed. For processes which are limited by statistics at the LHC, the SLHC may be useful. The increased luminosity of the sLHC could enable the observation of rare Higgs decays. The sLHC could also potentially increase the accuracy of the measurements of Higgs couplings. There might be some sensitivity on the tri-linear Higgs self-coupling [34]; however, some background contributions might have been underestimated. Further studies to clarify these issues are currently in progress, see Ref. [39] for a discussion. A key concern is maintaining detector performance, since the increased luminosity will result in significantly more pileup per beam crossing, increasing occupancy rates in the tracking systems.

ILC:

The following details are based on the Technical Design Report (TDR) that appeard in 2001 (for the TESLA design [40]), the Reference Design Report (RDR) [41] and subsequent documents (see also Ref. [42]). The initial stage of the ILC is expected to have an energy of $\sqrt{s}=500 \mathrm{GeV}$ with a luminosity of $2 \times 10^{34} / \mathrm{cm}^{2} / \mathrm{s}$, along with $90 \%$ polarization of the $e^{-}$beam and $30-45 \%$ polarization of the $e^{+}$beam. A future upgrade to $\sqrt{s}=1 \mathrm{TeV}$ (with an even higher luminosity) is envisioned. An advantage of the machine is that it is designed to have low beamstrahlung and a precise knowledge of the luminosity $\left(\delta \mathcal{L} / \mathcal{L}<10^{-3}\right)$ and energy $((\delta \sqrt{s}) / \sqrt{s}<200$ ppm), along with excellent detector resolution. The tunable energy scale allows for a scan of particle production thresholds.

The ILC offers a clean environment for the precision measurement of all quantum numbers and couplings of the Higgs boson, in addition to precision measurements of its mass and width. While the mass range of a SM-like Higgs boson can be covered completely by an ILC with $\sqrt{s}=$ $500 \mathrm{GeV}$ up to $M_{H} \lesssim 400 \mathrm{GeV}$, the achievable precision on the measurements of couplings and other properties is strongly dependent on the Higgs mass and differs for the various decay modes. A set of studies of properties of the Higgs boson has been collected in [40], many of which are being updated using the most recent designs for the accelerator and the detectors and fully simulated Monte Carlo events in [43] (see also Refs. [42,44]). A summary of the current analyses is given in Table 1.6 for $M_{H} \approx 120 \mathrm{GeV}$ and $M_{H} \approx 200 \mathrm{GeV}$. 


\begin{tabular}{|c|c|c|}
\hline Observable & Expected precision & Reference \\
\hline \multicolumn{3}{|c|}{ SM-like Higgs with $M_{H} \approx 120 \mathrm{GeV}$} \\
\hline$M_{H}[\mathrm{GeV}]$ & $0.04 \%$ & [40] \\
\hline$\Gamma_{H}[\mathrm{GeV}]$ & $0.056 \%$ & [40] \\
\hline$g_{H W W}$ & $1.2 \%$ & [40] \\
\hline$g_{H Z Z}$ & $1.2 \%$ & [40] \\
\hline$g_{H t t}$ & $3.0 \%$ & [40] \\
\hline$g_{H b b}$ & $2.2 \%$ & [40] \\
\hline$g_{H c c}$ & $3.7 \%$ & [40] \\
\hline$g_{H \tau \tau}$ & $3.3 \%$ & [40] \\
\hline$g_{H t t}$ & $7 \%$ & [45] \\
\hline$g_{H H H}$ & $22 \%$ & [40] \\
\hline $\mathrm{BR}(H \rightarrow \gamma \gamma)$ & $23 \%$ & [40] \\
\hline $\mathcal{C P}{ }_{H}$ & $4.7 \sigma$ diff. between even and odd & [46] \\
\hline GigaZ Indirect $M_{H}[\mathrm{GeV}]$ & $7 \%$ & {$[47,48]$} \\
\hline \multicolumn{3}{|c|}{ Heavy SM-like Higgs with $M_{H} \approx 200 \mathrm{GeV}$} \\
\hline$M_{H}[\mathrm{GeV}]$ & $0.11 \%$ & [49] \\
\hline $\operatorname{direct} \Gamma_{H}[\mathrm{GeV}]$ & $34 \%$ & [49] \\
\hline $\mathrm{BR}(H \rightarrow W W)$ & $3.5 \%$ & [49] \\
\hline $\mathrm{BR}(H \rightarrow Z Z)$ & $9.9 \%$ & [49] \\
\hline $\mathrm{BR}(H \rightarrow b \bar{b})$ & $17 \%$ & [50] \\
\hline$g_{H t t}$ & $14 \%$ & [45] \\
\hline \multicolumn{3}{|c|}{ Additional Measurements for Non-SM Higgs with $M_{H} \approx 120 \mathrm{GeV}$} \\
\hline $\mathrm{BR}(H \rightarrow$ invisible $)$ & $<20 \%$ for $\mathrm{BR}>0.05$ & [40] \\
\hline
\end{tabular}

Table 1.6: Examples of the precision of SM-like Higgs observables at a $\sqrt{s}=500 \mathrm{GeV}$ ILC. For the direct measurements, an integrated luminosity of $\mathcal{L}^{\text {int }}=500 \mathrm{fb}^{-1}$ is assumed (except for the $b \bar{b}$ channel at $M_{H} \approx$ $200 \mathrm{GeV}$ and the $t \bar{t}$ channel, which assume $\sim 1 \mathrm{ab}^{-1}$ at $\sqrt{s}=800 \mathrm{GeV}$ ). For the indirect measurements at GigaZ, a running time of approximately one year is assumed, corresponding to $\mathcal{L}=\mathcal{O}\left(10 \mathrm{fb}^{-1}\right)$.

In addition, the options of GigaZ $\left(10^{9} Z\right.$ 's at $\left.\sqrt{s} \approx M_{Z}\right)[47,51]$, and MegaW $(\sqrt{s} \approx$ $2 M_{W}$ ) [52] allow precision tests of the SM with uncertainties reduced approximately by one order of magnitude from the predictions of current ILC studies. This would allow the mass of the SM Higgs boson to be constrained quite strongly by indirect methods and could potentially exclude a SM-like Higgs boson with $M_{H} \gtrsim 130 \mathrm{GeV}$ [47,48] (see Ref. [53] for a corresponding MSSM analysis).

Another ILC option are $e \gamma$ or $\gamma \gamma$ collisions (the Photon Linear Collider, PLC) [54], with $\gamma$ beams obtained from the backscattering on laser beams. The energy of the photons would be 


\begin{tabular}{|l||c|c|c|c|}
\hline coupling $/ M_{H}[\mathrm{GeV}]$ & 120 & 150 & 180 & 220 \\
\hline \hline$g_{H b b}$ & & & $1.6 \%$ & $3.4 \%$ \\
$g_{H \mu \mu}$ & $4.2 \%$ & $11.0 \%$ & & \\
$g_{H H H}$ & $9.3 \%$ & & $11.5 \%$ & \\
\hline
\end{tabular}

Table 1.7: Examples of the precision, $\delta g_{H x x} / g_{H x x}$, for measurements of Higgs couplings at a $\sqrt{s}=3 \mathrm{TeV}$ CLIC with $3 \mathrm{ab}^{-1}$ [55].

$\sim 80 \%$ of the electron beam, maintaining a high degree of polarization and a luminosity of $\sim 1 / 3$ of the ILC in the high energy peak. The PLC could potentially perform precision measurements of resonantly produced Higgs bosons. Combining ILC and PLC measurements, the $H \gamma \gamma$ coupling could be determined at the level of $\sim 3 \%$.

CLIC:

$\overline{\mathrm{CLIC}}$ is proposed as a multi-TeV $e^{+} e^{-}$collider with an energy of $\sqrt{s} \sim 1-3 \mathrm{TeV}$ and a luminosity of $\mathcal{L} \sim 10^{34} / \mathrm{cm}^{2} / \mathrm{s}$. The goal of the current studies is to demonstrate technical feasibility and to have a Conceptual Design Report by 2010 and a Technical Design Report by 2015. Examples of anticipated precisions for Higgs boson couplings are given in Table 1.7. Analyses mostly focused on channels that are challenging at the ILC, see Ref. [55] and references therein.

\subsubsection{Scenario A: SM-like Higgs with $130 \mathrm{GeV} \leq M_{H} \leq 180 \mathrm{GeV}$}

In the region of masses $130-180 \mathrm{GeV}$, the dominant decay modes considered are to two vector bosons, $Z Z^{(*)}$ and $W W^{(*)}$, yielding a discovery at the $\mathrm{LHC}_{10 / \mathrm{fb}}$. New studies [7] indicate that even $200 \mathrm{pb}^{-1}$ could be sufficient to probe the region of $160 \mathrm{GeV} \leq M_{H} \leq 170 \mathrm{GeV}$, which is currently excluded at $95 \%$ C.L. at the Tevatron [1].

For $M_{H} \lesssim 200 \mathrm{GeV}$, the total Higgs width cannot be measured at the LHC and there is expected to be only an upper limit of $\mathcal{O}(1 \mathrm{GeV})$. Hence in this mass region, only ratios of Higgs couplings can be measured in a model independent fashion, see Table 1.5 for the $\mathrm{LHC}_{10 / \mathrm{fb}}$ expectations. In the lower part of the mass range, $130 \mathrm{GeV} \leq M_{H} \leq 150 \mathrm{GeV}$, the $\gamma \gamma$ and $\tau^{+} \tau^{-}$final states are accessible. As discussed above, the final state with $b$ quarks $(H \rightarrow b \bar{b})$ seems not to be accessible during the first years of the LHC, because of the very difficult background environment.

In this scenario the large mass reach of the sLHC could be helpful to detect new scales beyond the SM. Furthermore, the sLHC can in principle improve the accuracy of Higgs coupling constant measurements in this regime [23]. For $M_{H} \gtrsim 150 \mathrm{GeV}$, the decays $H \rightarrow Z Z^{*} \rightarrow 4 l$ and $H \rightarrow W^{+} W^{-} \rightarrow l \nu l \nu$ provide a direct measurement of the ratio of the partial Higgs widths, $\Gamma_{H Z Z} / \Gamma_{H W W}$. For $M_{H}=170 \mathrm{GeV}, 3000 \mathrm{fb}^{-1} /$ experiment could improve the measurement of $\delta\left(\Gamma_{H Z Z} / \Gamma_{H W W}\right) /\left(\Gamma_{H Z Z} / \Gamma_{H W W}\right)$ from the $\mathrm{LHC}_{300 / \mathrm{fb}}$ measurement by about a factor of 1.5 . The improvement at the sLHC for most other masses values in the intervall $M_{H}=150-180 \mathrm{GeV}$ is quite small, however. In this mass region, the decays $H \rightarrow \tau^{+} \tau^{-}$and $H \rightarrow W^{+} W^{-} \rightarrow l \nu l \nu$ provide a direct measurement of $\Gamma_{H \tau \tau} / \Gamma_{H W W}$. The improvement in this channel at the sLHC over the $\mathrm{LHC}_{300 / \mathrm{fb}}$ result is not known, since it depends crucially on $\tau$ identification, missing $E_{T}$ capabilities, and identification of forward jets at $\mathcal{L}=10^{35} / \mathrm{cm}^{2} / \mathrm{s}$. 
The sLHC is sensitive to rare Higgs decays for light Higgs bosons [23]. The decay $H \rightarrow$ $\mu^{+} \mu^{-}$has a branching ratio $\sim 10^{-4}$ and almost certainly cannot be observed at the $\mathrm{LHC}_{300 / \mathrm{fb}}$. For $M_{H}=140 \mathrm{GeV}$, the sLHC can obtain a $5.1 \sigma$ observation and an accuracy of $\delta(\sigma \times \mathrm{BR}(H \rightarrow$ $\left.\left.\mu^{+} \mu^{-}\right)\right) /\left(\sigma \times \mathrm{BR}\left(H \rightarrow \mu^{+} \mu^{-}\right)\right)=0.2$ with $3000 \mathrm{fb}^{-1}$ /experiment. The accuracy rapidly decreases with increasing Higgs mass and for $M_{H}=150 \mathrm{GeV}$ the significance is $2.8 \sigma$ with an accuracy of $\delta\left(\sigma \times \operatorname{BR}\left(H \rightarrow \mu^{+} \mu^{-}\right)\right) /\left(\sigma \times \operatorname{BR}\left(H \rightarrow \mu^{+} \mu^{-}\right)\right)=0.36$. Similarly, the decay $H \rightarrow Z \gamma$ can be observed at $11 \sigma$ for $100 \mathrm{GeV} \leq M_{H} \leq 160 \mathrm{GeV}$. This is to be compared with $3.5 \sigma$ at $\mathrm{LHC}_{300 / \mathrm{fb}}$.

Since in this scenario the coupling of the Higgs to $Z Z$ and/or $W^{+} W^{-}$will have been observed at the $\mathrm{LHC}_{10 / \mathrm{fb}}$, the production of this new state via $e^{+} e^{-} \rightarrow Z^{*} \rightarrow Z H$ or $e^{+} e^{-} \rightarrow$ $\nu \bar{\nu} W^{+} W^{-} \rightarrow \nu \bar{\nu} H$ at lepton colliders is guaranteed. Lepton colliders can potentially improve the precision measurements of the Higgs couplings, self-coupling, width and spin in this Higgs mass region. At the ILC, the total cross section can be measured in a decay mode independent analysis via $Z$ recoil in the channel $e^{+} e^{-} \rightarrow Z H$, from which, in conjunction with the branching fractions, the absolute values of the couplings can be derived. For $M_{H}<150 \mathrm{GeV}$, a precision measurement of the absolute values of the Higgs boson couplings to $W, b, \tau, c, t$ and $g, \gamma$ (through loops, possibly combining with the PLC option) can be achieved [40], see Table 1.6. The mass can be measured with a precision of around $\Delta M_{H} \approx 50 \mathrm{MeV}$. In addition, the $\mathcal{C P}$ quantum numbers can be measured in $\tau$ decays [46] and the spin can be determined both in production and in decay. For $\sqrt{s}=800-1000 \mathrm{GeV}$, the $t \bar{t} H$ associated production allows the measurement of the coupling to the top quark [45], and for very high luminosities also the Higgs self-coupling can be measured in Z H H final states [56]. Thus, a nearly complete precise Higgs boson profile could be determined, and possible signals of scales beyond the SM could be detected.

For $M_{H}=160-180 \mathrm{GeV}$ (currently probed by the Tevatron Higgs searches), the Higgs decays are dominated by $H \rightarrow W^{+} W^{-}$, suppressing the branching fractions of the Higgs into most of the particles mentioned above below the per-mille range, making precision measurements of those couplings impossible with $\sqrt{s}=500 \mathrm{GeV}$. Besides the decay to vector bosons, the $b \bar{b}$ channel remains a relatively precise observable at the ILC. Consequently an important part of the Higgs profile could be determined in a model-independent way (including as well possible exotic or invisible decay channels). Also at CLIC the detectable channels remain the same, see Table 1.7. In this way these colliders could be sensitive to new scales beyond the SM.

In this Higgs mass range, on the other hand, the ILC with the GigaZ $\left(\sqrt{s} \approx M_{Z}\right)$ and MegaW $\left(\sqrt{s} \approx 2 M_{W}\right.$ ) options could indirectly exclude a SM Higgs, based on precision observables [47, 48 ], at more than $3 \sigma$ throughout (if the order of magnitude of the current measurements of the precision observables is in the right range). Given the possible precision, $M_{H} \gtrsim 130 \mathrm{GeV}$ could be excluded in the SM at the $3 \sigma$ level if the true SM Higgs mass stays at its current best fit point of around $M_{H} \approx 90 \mathrm{GeV}$. The combination of Higgs observables and more precise SM observables would offer new realms for the precision tests of New Physics theories explaining the apparent difference between precision observables and an observed Higgs mass above $\sim 160 \mathrm{GeV}$.

\subsubsection{Scenario B-I: SM-like Higgs with $M_{H} \leq 130 \mathrm{GeV}$}

In the region of low masses, $114 \mathrm{GeV} \leq M_{H} \leq 130 \mathrm{GeV}$, a channel where the LHC has the potential to discover the Higgs at the $5 \sigma$ level is the $H \rightarrow \gamma \gamma$ final state. The CMS optimized

analysis shows a discovery potential in this region with $\sim 10 \mathrm{fb}^{-1}$. Close to $M_{H}=130 \mathrm{GeV}$ the 
channel $H \rightarrow Z Z^{*} \rightarrow 4$ leptons could also reach the $5 \sigma$ level. On the other hand, in the mass region $M_{H} \lesssim 130 \mathrm{GeV}$ effects smaller than $5 \sigma$ could be studied separately for the vector boson fusion and $g g$ production channels. The $q q \rightarrow q q H, H \rightarrow \tau^{+} \tau^{-}$channel can reach only the 2 to $3 \sigma$ level.

Rare decays for a light Higgs can be studied at the sLHC. For example, for $M_{H}=120 \mathrm{GeV}$, the sLHC can obtain a $7.9 \sigma$ observation of the $\mu \mu$ channel and an accuracy of $\delta(\sigma \times \mathrm{BR}(H \rightarrow$ $\left.\left.\mu^{+} \mu^{-}\right)\right) /\left(\sigma \times \operatorname{BR}\left(H \rightarrow \mu^{+} \mu^{-}\right)\right)=0.13$ with $3000 \mathrm{fb}^{-1}$ /experiment.

For the ILC, even running at $\sqrt{s}$ substantially below $500 \mathrm{GeV}$, the range $M_{H} \lesssim 130 \mathrm{GeV}$ should be particularly "easy". The ILC will be able to measure many Higgs properties in the light Higgs mass range: the mass, couplings (in a model-independent way) to nearly all fermions of the third family, to the SM gauge bosons and (running at $\sqrt{s}=800 \mathrm{GeV}$ ) the tri-linear Higgs self-couplings. Also a determination of the Higgs boson spin and quantum numbers should be easily feasible. The anticipated precisions are summarized in Table 1.6. Thus, a nearly complete Higgs boson profile could be determined, and possible signals of scales beyond the SM could be detected.

CLIC operating at $\sqrt{s}=3 \mathrm{TeV}$ can observe many Higgs boson decays. In addition to what could be measured at the ILC, particularly interesting would be the Higgs decay $H \rightarrow \mu^{+} \mu^{-}$ through the vector boson fusion process, $e^{+} e^{-} \rightarrow H \nu \bar{\nu}$ [57]. With $5 \mathrm{ab}^{-1}$, CLIC can obtain a precision on the coupling constant of $\delta g_{H \mu \mu} / g_{H \mu \mu}=0.04$ for $M_{H}=120 \mathrm{GeV}$. CLIC can also obtain a $10 \%$ measurement of $g_{H H H}$ for $M_{H}=120 \mathrm{GeV}$.

\subsubsection{Scenario B-II: SM-like Higgs with $M_{H} \geq 180 \mathrm{GeV}$}

This region is severely constrained by the electroweak precision fits $[48,58,59]$. Excluding the direct search results, the $3 \sigma$ allowed region is $M_{H} \leq 209 \mathrm{GeV}$. When the direct search results from LEP2 and the Tevatron are included, the $3 \sigma$ allowed region is $M_{H} \leq 168 \mathrm{GeV}$ or $180 \mathrm{GeV} \leq$ $M_{H} \leq 225 \mathrm{GeV}$. Consequently, the discovery of a Higgs boson above this mass range, even in the absence of any other signal, would point to new physics beyond the SM.

The main channel for the Higgs discovery at the LHC in this mass region is $H \rightarrow Z Z \rightarrow 4 l$ and with only $2 \mathrm{fb}^{-1}$ the mass range $M_{H} \sim 190-500 \mathrm{GeV}$ can be covered. Starting at a luminosity around $30 \mathrm{fb}^{-1}$, the vector boson fusion Higgs production channel can be studied and Higgs masses up to $1 \mathrm{TeV}$ can be explored using $H \rightarrow W^{+} W^{-} \rightarrow l l q q$.

With $300 \mathrm{fb}^{-1}$, spin-parity quantum numbers $0^{-+}$and $1^{--}$can be excluded for $M_{H}=$ $230 \mathrm{GeV}$ [38]. The total width can be measured to a precision of $35 \%$ with $30 \mathrm{fb}^{-1}$ from $H \rightarrow$ $Z Z^{*} \rightarrow 4$ leptons above $M_{H} \sim 200 \mathrm{GeV}$ [3] and ultimately to $5-8 \%$ with $300 \mathrm{fb}^{-1}$ [60].

In the mass range $170 \mathrm{GeV} \leq M_{H} \leq 200 \mathrm{GeV}$, the sLHC may be able to observe Higgs boson pair production through the process $g g \rightarrow H H \rightarrow l^{+} l^{\prime+} 4 j$, thus getting sensitivity for the tri-linear Higgs self-coupling. A critical feature of the analysis is the assumption that detector capabilities at the sLHC are roughly the same as at the LHC. Further studies to clarify these issues are currently in progress, as discussed in Ref. [39].

As above, the precision SM observables of the ILC GigaZ and MegaW options could indirectly rule out this mass range in the SM. On the other hand, the precision measurement of the Higgs mass, the couplings to the top, $W$ and $Z$ and the direct measurement of the Higgs width [49] 
from the lineshape would be possible at the ILC if the Higgs boson is kinematically accessible. These measurements will yield stringent constraints on potential New Physics models explaining the high Higgs boson mass and point to new scales beyond the SM.

\subsubsection{Scenario C: A non-SM-like Higgs}

Another possibility at the $\mathrm{LHC}_{10 / \mathrm{fb}}$ would be to observe a state compatible with a Higgs boson that appears to be in disagreement with the SM predictions. This could be due to an $M_{H}$ value outside the region allowed by the precision data. A mass above $\sim 170 \mathrm{GeV}$ would indicate a disagreement with today's precision data (not taking into account the direct searches, see above) [59]. We will not pursue this option further and assume for the rest of the discussion a mass in the range $110 \mathrm{GeV} \lesssim M_{H} \lesssim 160 \mathrm{GeV}$.

Another possibility for a non-SM-like Higgs boson would be production and/or decay rates different from the SM prediction. While a suppression of a decay could only be observed in the sensitive channels, a strong enhancement could appear in any of the search channels (and is consequently more arbitrary). It is possible to suppress all of the Higgs couplings by the simple mechanism of adding extra Higgs singlets, which can make the Higgs search at the LHC quite challenging $[61,62]$.

Of particular interest are the loop-mediated Higgs couplings, $H \rightarrow \gamma \gamma$ and $H \rightarrow g g$, which can receive sizable contributions from New Physics (NP). In many NP models the Higgs couplings to $W$ and $Z$ remain essentially unaffected. Several channels at the LHC are sensitive to different combinations of these loop-mediated Higgs couplings, which allows a quasi-model independent analysis [63], potentially shedding light on the nature of new states discovered at the LHC and on the underlying model of electroweak symmetry breaking. The simultaneous measurement of the inclusive $H \rightarrow \gamma \gamma$ and $H \rightarrow W^{+} W^{-}$cross sections, as well as the vector boson fusion process, $q q \rightarrow q q H, H \rightarrow \gamma \gamma$ cross section, allows the placement of constraints in the two-dimensional plane of the $H \gamma \gamma$ and $H g g$ couplings. From a survey of NP models performed in Ref. [63] contributions to some of these cross sections as large as 50\% were found (see also Ref. [64] for a 2HDM analysis). Therefore, measurements of these cross sections at the LHC with 10-20\% accuracy should allow some discrimination of NP models. At the ILC, the percent-level measurement of the $H \rightarrow \gamma \gamma$ and $H \rightarrow W W$ branching ratios will allow a much more sensitive probe of NP models.

For $M_{H} \lesssim 130 \mathrm{GeV}$ a channel suppression at the $\mathrm{LHC}_{10 / \mathrm{fb}}$ would only be possible in the decay $H \rightarrow \gamma \gamma$, which could be due to either a suppressed $H W W$ coupling (or a large new loop correction interfering negatively with the $W$ loop contribution) or an enhancement of a Higgs branching ratio to a channel invisible at the $\mathrm{LHC}_{10 / \mathrm{fb}}$. Invisible could mean the Higgs boson decays either to known particles that are difficult to detect at the LHC, such as decays to light quarks, or to truly invisible particles such as neutrinos, the lightest SUSY particle (assuming $R$ parity conservation), the lightest Kaluza-Klein mode etc. A sensitivity at the $\mathrm{LHC}_{10 / \mathrm{fb}}$ to invisible decays (assuming SM production rates) would only be possible if the BR into the invisible channel is close to $100 \%$ [65] (see also WG2 report).

For $130 \mathrm{GeV} \lesssim M_{H} \lesssim 170 \mathrm{GeV}$ a suppression of the decays to $W W^{(*)}$ and/or $Z Z^{(*)}$ could be observable. As for the light Higgs case this could be due to either a suppressed $H W W$ and/or $H Z Z$ coupling or an enhancement of a Higgs branching ratio to a channel invisible at the 
$\mathrm{LHC}_{10 / \mathrm{fb}}$.

A measurement of decays that are suppressed due to the (enhancement of the) decay to difficult or invisible channels at the $\mathrm{LHC}_{10 / \mathrm{fb}}$ would improve with higher luminosity. The sensitivity could (assuming SM Higgs production rates) go down to a BR of $\sim 15 \%$ [65]. Consequently, the observation of a suppression could be backed-up by the observation of "invisible" decays.

At the ILC, see Table 1.6, any kinematically accessible decay channel with a substantial decay rate will be detectable, including the decay to truly invisible particles. Therefore at the ILC a nearly complete Higgs boson profile could be determined. The ILC would be ideal to shed light on this case. Similar results could be expected for CLIC.

A suppressed coupling of the Higgs to vector bosons would strongly hint towards an extended Higgs sector where several Higgs bosons share the couplings to the $W^{+} W^{-}$and $Z Z$. A maximum coverage of the mass range would be needed to discover these additional Higgs bosons (or to reject this solution). The LHC could cover masses up to $\lesssim 1 \mathrm{TeV}$ assuming SM-like decays. The situation improves slightly at the sLHC. The ILC (especially with the $\gamma \gamma$ option [54]) would have a good chance to discover heavier Higgs bosons (with $M_{H} \lesssim 400 \mathrm{GeV}$ ) also with non-SM-like decay rates. CLIC, with the high $\sqrt{s} \approx 3 \mathrm{TeV}$ would cover an even larger Higgs mass range.

This search could be supplemented by the analysis of $W W$ scattering at very high energies to investigate whether other forces than the Higgs mechanism might be at work. In this way the measurement of the cross section of $W W$ scattering as a function of the invariant mass of the di-boson is a key ingredient to the understanding of the symmetry breaking mechanism. Within the SM the Higgs particle is essential to the renormalization of the theory and ensures that the unitarity bounds are not violated in high energy interactions, i.e. $\sigma(W W \rightarrow W W)$ does not rise with $M(W W)$ for $M(W W) \gtrsim 1-2 \mathrm{TeV}$, and a resonance at $M(W W)=M_{H}$ should be observable. If another mechanism of electroweak symmetry breaking is realized in nature, see e.g. [66] and references therein, the behavior of $\sigma(W W \rightarrow W W)$ will deviate from the SM expectations. Corresponding LHC studies can be found in [2] (p. 1695) and [67, 68]. Both ATLAS and CMS expect to be able to discover strongly interacting resonances with $M(W W)>1 \mathrm{TeV}$ only with $100 \mathrm{fb}^{-1}$ or more. Integrated luminosities of $300-500 \mathrm{fb}^{-1}$ will be necessary to understand the shape of the $\sigma(W W \rightarrow W W)$ vs. $M(W W)$ distribution at high energies in order to investigate if a light Higgs is present or a different mechanism of electroweak symmetry breaking is realized. In order to explore this region fully, the sLHC will be necessary. At the ILC new resonance scales up to $\sim 30 \mathrm{TeV}$ could be probed indirectly [44,66], while at CLIC direct resonances up to $\sim 2.5 \mathrm{TeV}$ could be accessed (for $\sqrt{s}=3 \mathrm{TeV}$ ) [55]. More details can be found in Sect. 2 .

There are of course, many possibilities for non-SM Higgs bosons, such as scenarios with multiple Higgs bosons or with very light Higgs bosons which evade the LEP Higgs searches. Some of these options are discussed in the Sect. 2.

\subsection{Summary and conclusions of WG1}

The LHC will explore the mechanism responsible for electroweak symmetry breaking. Assuming that a new state as a possible candidate for a Higgs boson will have been observed at the $\mathrm{LHC}_{10 / \mathrm{fb}}$, the full identification of the mechanism of electroweak symmetry breaking will require to measure all its characteristics. This comprises an accurate mass determination, a (model-independent) 
measurement of its individual couplings to other particles, a determination of the self-couplings to confirm the "shape" of the Higgs potential, as well as measurements of its spin and $\mathcal{C P}$-quantum numbers . At the LHC, even running at high luminosity, these measurements will only partially be possible.

We reviewed what we might know about the Higgs sector once the LHC has collected $10 \mathrm{fb}^{-1}$ (of understood data) at a center-of-mass energy of $14 \mathrm{TeV}$. Based on the anticipated future knowledge of the Higgs sector, we investigated the capabilities of future colliders to further unravel the Higgs mechanism.

While the sLHC will be able to extend the reach and precision of the $\mathrm{LHC}_{300 / \mathrm{fb}}$ it seems clear that a full exploration of the Higgs sector will require either the ILC or CLIC. For a SM-like Higgs with $M_{H} \lesssim 150 \mathrm{GeV}$ at the ILC a nearly complete Higgs boson profile could be determined. For larger masses (currently probed at the Tevatron) the decay to SM gauge bosons becomes dominant, suppressing other decay modes and making them more difficult to measure. In the case of a non-SM-like Higgs nearly all channels visible at the ILC can be determined with high accuracy. The corresponding CLIC analyses have focused mostly on measurements that are challenging at the ILC. Due to its high luminosity and high center-of-mass energy up to $\sqrt{s} \approx 3 \mathrm{TeV}$ very heavy Higgs bosons, for instance from extended Higgs sectors, could be probed. The precision measurements obtainable at the ILC and CLIC could point to New Physics beyond the SM, opening the window to energy scales beyond the LHC. 


\section{WG2: No Higgs boson}

G. Azuelos, C. Grojean, M. Lancaster, G. Weiglein (convenors)

S. Dawson, S. De Curtis, M.T. Frandsen, R. Godbole, P. Govoni, J. Gunion, T. Han, S. Heinemeyer,

G. Isidori, A. Martin, E. Ozcan, T. Plehn, F. Sannino, M. Schram

If no Higgs candidate is found in the first $10 \mathrm{fb}^{-1}$ at the LHC, two options will have to be considered: (i) A Higgs boson exists (or more than one) but it has non-standard properties that make it difficult to detect because of suppressed couplings to gauge bosons and/or fermions or because of unusual decays; or (ii) There really is no fundamental Higgs boson and new degrees of freedom or new dynamics beyond the Standard Model are needed to maintain unitarity at high energy. The implications of these two scenarios for future colliders are discussed in this section.

\subsection{Introduction}

The exploration of the Terascale at the LHC will probe directly the dynamics responsible for electroweak symmetry breaking (EWSB). While the evidence in favour of spontaneous breaking of the electroweak symmetry is very strong, the fact that this breaking occurs via a single fundamental Higgs field, with a non-trivial vacuum expectation value, is far from being clearly established. The Higgs mechanism is certainly the most economical way of explaining this spontaneous breaking, and a light Higgs mass $\left(m_{h} \approx 100 \mathrm{GeV}\right.$ ) is also an efficient way to account for all the existing electroweak precision tests (EWPTs). However, the strong sensitivity of $m_{h}$ to short-distance scales poses a serious naturalness problem at least for the Standard Model (SM) and motivates the search for alternative symmetry-breaking mechanisms. The SM Higgs boson plays the role of moderator of the strength of longitudinal $W$ interactions and allows the model to be extrapolated to very short distances. Thus in the absence of a Higgs boson, the dynamics behind EWSB is expected to become strong around $\mathrm{a} \mathrm{TeV}$ and to deviate significantly from the SM.

In this report we consider the situation where a Higgs-like signal is absent in the early LHC data and we investigate in how far the possible physics scenarios can be constrained in such a case. The non-observation of a Higgs candidate in the first $10 \mathrm{fb}^{-1}$ at the LHC could evidently point to one of the two following options:

- There exists a Higgs boson (or more than one) but it has non-standard properties that make it difficult to detect, either because of suppressed couplings to gauge bosons and/or fermions, or because of unusual decays.

- There really is no fundamental Higgs boson. Then new degrees of freedom or new dynamics beyond the Standard Model are required to maintain unitarity at high energy.

In the case of the absence of a Higgs-like signal in the early LHC data it is of particular importance to investigate the behavior of the $W_{L} W_{L}$ scattering amplitudes, which will be directly affected by the dynamics responsible for restoring unitarity. 


\subsection{Unobserved Higgs boson scenarios}

With $10 \mathrm{fb}^{-1}$, a truly SM-like Higgs boson will be discovered by ATLAS and CMS, since the LHC covers the full kinematic range expected for a SM Higgs boson from unitarity arguments and EWPTs (see WG1 summary). However, for a Higgs boson with non-standard properties it could be more difficult to extract a Higgs signal from the data in the experimental environment at the LHC. Thus, if the Higgs mechanism is realized in nature, the absence of a conclusive sign of a Higgs boson at the $\mathrm{LHC}_{10 / \mathrm{fb}}$ would point towards a Higgs sector with a more involved structure than in the SM.

In models with an extended Higgs sector and/or an enlarged particle content Higgs phenomenology can drastically differ from the SM case. On the one hand, it should be recalled that the SM exclusion bound obtained at LEP of $m_{h}^{\mathrm{SM}}>114.4 \mathrm{GeV}$ at $95 \%$ C.L. [11] is not applicable for a more complicated Higgs sector giving rise to a suppression of the coupling of a light Higgs to gauge bosons and possibly also yielding unusual decay properties. Thus, the possibility that a Higgs boson much lighter than the SM exclusion bound has escaped detection in the searches carried out up to now cannot be excluded. Such a light Higgs boson could be very difficult to detect also at the LHC. A well-known realisation of such a scenario is the Minimal Supersymmetric Standard Model (MSSM) with non-vanishing complex phases in the CPX benchmark scenario [69] giving rise to a Higgs boson as light as about $45 \mathrm{GeV}$ that is unexcluded by the LEP searches [12]. The Higgs bosons in this scenario would also be difficult to detect with the standard search channels at the LHC [70-72].

On the other hand, the lightest Higgs boson could also be much heavier than the mass range preferred for a SM-type Higgs by the EWPTs. In this case new physics contributions to electroweak precision observables would be necessary to compensate the effects of a heavy Higgs boson, mimicking in this way the contribution from a light SM-like Higgs boson.

Even if there exists a light Higgs boson not far above the exclusion bound for a SM-type Higgs, its properties could still be very different from a SM Higgs. In the SM scenario, a light Higgs boson with a mass below the $W W$ threshold has a rather narrow decay width as the heaviest SM particles it can decay to is a $b$ quark pair. The $H b b$ coupling is quite small, only about $1 / 40$. As a consequence, any new particle with less than half the Higgs mass which interacts with the Higgs boson could modify the decay branching fractions very substantially. If these new particles carry no colour or electroweak charge then they will be difficult to produce directly at the LHC. However, they are likely to alter Higgs decays, leading to final states that could be either visible but complicated or invisible. An example of the latter is Higgs decays to a pair of LSPs in supersymmetric models. In this case, the LEP lower bound on the mass of a Higgs boson with SM-like $W W, Z Z$ couplings is $114 \mathrm{GeV}$. An example of the former is Higgs decays to a pair of light CP-odd scalars that are primarily SM singlets, as strongly motivated in the Next to Minimal Supersymmetric Model (NMSSM) [73,74]. In this case, the LEP lower bound on the Higgs mass could be as small as $82 \mathrm{GeV}$.

Couplings in the Higgs sector can differ from the SM case both because of a different treelevel structure and because of higher-order contributions that can often be very large. In the MSSM, for instance, the couplings of neutral Higgs bosons to a pair of down-type fermions can be very strongly enhanced for large values of the parameter $\tan \beta$, giving rise to a simultaneous suppression of the branching ratios into $\gamma \gamma, W W^{(*)}, Z Z^{(*)}$. Higgs production in gluon fusion can be 
suppressed if there is a destructive interference of the top-quark loop with the contributions from other new particles, like the superpartners of the top quark in the case of the MSSM (see e.g. Refs. $[66,75,76])$.

Higgs properties can also be modified very significantly if a Higgs boson mixes with other states of new physics. An example for such a scenario is the mixing of a Higgs boson with a radion, a state that is predicted in models with 3-branes in extra dimensions. As the radion has the same quantum numbers as the Higgs boson the two states will mix with each other in general. Since the radion has couplings that are different from those of the SM Higgs boson, the two physical eigenstates will have unusual properties that differ substantially from the ones of the pure Higgs state [77].

The impact of new physics contributions in the Higgs sector can, on the one hand, be to enhance the prospects for Higgs searches, for instance by opening up new discovery channels, while, on the other hand, unusual Higgs properties can also make it much more difficult to extract a Higgs signal from the data than is the case for a SM Higgs. Examples that could lead to a situation where no Higgs signal can be established at least with the first $10 \mathrm{fb}^{-1}$ at the LHC are the case of a Higgs boson that decays primarily into hadronic jets, possibly without definite flavour content, or the possibility that a Higgs boson with SM-type couplings could primarily decay into a pair of very light pseudoscalar Higgs bosons. Another very difficult scenario for Higgs boson detection would be the case of a "continuum" Higgs model, i.e. a large number of doublet and/or singlet fields with complicated self interactions (see, e.g., Refs. [62, 78, 79]). This could result in a very significant diminution of all the standard LHC signals.

A review of phenomenological consequences of non-standard Higgs boson decays can be found in Ref. [80]. Table 2.1 gives the 95\% C.L. lower LEP limits on $m_{h}$ for a Higgs boson assuming that it has SM-like couplings to gauge bosons, obtained from searches in specific channels.

\begin{tabular}{|c|c|c|c|c|c|c|c|c|c|c|}
\hline Decay mode & SM & $2 \tau, 2 b$ & $2 \mathrm{j}$ & $W W^{\star}$ & $\gamma \gamma$ & $\mathbb{E}$ & $4 e, 4 \mu, 4 \gamma$ & $4 b$ & $4 \tau$ & anything \\
\hline \hline$m_{h}$ bound $[\mathrm{GeV}]$ & 114.4 & 115 & 113 & 109.7 & 117 & 114 & 114 & 110 & 86 & 82 \\
\hline
\end{tabular}

Table 2.1: LEP bounds on the Higgs mass for a Higgs boson with SM-like couplings to gauge bosons, as obtained from searches in specific decay modes (for a discussion of the assumptions used for obtaining these limits, see Ref. [11, 12,80] and references therein).

The main discovery channels for a light SM-like Higgs boson at the $\mathrm{LHC}_{10 / \mathrm{fb}}$ are $[2,3]$,

$$
\begin{aligned}
& m_{h} \lesssim 140 \mathrm{GeV}: q \bar{q} H, H \rightarrow \tau^{+} \tau^{-}, p p \rightarrow H, H \rightarrow \gamma \gamma \\
& 130 \mathrm{GeV} \lesssim m_{h}: q \bar{q} H, H \rightarrow W W^{(*)}, Z Z^{(*)} \\
& 140 \mathrm{GeV} \lesssim m_{h}: p p \rightarrow H+X, H \rightarrow Z Z^{(*)} \\
& 150 \mathrm{GeV} \lesssim m_{h}: p p \rightarrow H+X, H \rightarrow W W^{(*)}
\end{aligned}
$$

The ATLAS and CMS sensitivities in the search of the Higgs boson can be significantly affected in the low mass region for the following two main reasons: (i) the SM Higgs boson is dominantly produced by gluon fusion, a one-loop process that can receive large negative corrections from new physics, such as from squarks $[66,75,76]$ and (ii) the narrow width of the Higgs 
boson makes it more vulnerable to any new decay channels. It should be noted that if any non-zero coupling of the Higgs boson to gauge bosons can be observed at $\mathrm{LHC}_{10 / \mathrm{fb}}$, this would guarantee the Higgs production at the ILC via Higgs-strahlung or weak boson fusion. In this case a determination of the Higgs boson properties can be performed (see WG1 summary). Even if there are multiple mixed Higgs bosons which overlap within the mass resolution and which decay in multiple ways in such a way that no LHC signal is seen, the ILC would be guaranteed to see an enhancement in the $M_{X}$ distribution in $e^{+} e^{-} \rightarrow Z X$ and be able to study the decays of the individual Higgs bosons [79].

In the following we briefly discuss three examples of scenarios that could give rise to the absence of a clear Higgs signal in the first $10 \mathrm{fb}^{-1}$ at the LHC: (i) a light Higgs boson with reduced gauge couplings in the MSSM with non-vanishing complex phases; (ii) a light Higgs boson with full-strength gauge couplings but unusual decays; and (iii) a light Higgs boson with invisible decays.

\subsubsection{A light gaugephobic Higgs boson in the MSSM with complex parameters}

The Higgs sector of the MSSM appears to be a "minimal" extension of the SM Higgs sector in the sense that is characterized by two free parameters at lowest order (conventionally chosen as $\tan \beta$, the ratio of the two vacuum expectation values, and either the mass of the pseudoscalar Higgs boson, $m_{A}$, or the mass of the charged Higgs bosons, $m_{H^{ \pm}}$) instead of the single free parameter in the case of the SM (the mass of the Higgs boson). Nevertheless the different tree-level structure can change Higgs phenomenology very substantially. Furthermore, large higher-order effects also play an important role.

While the MSSM Higgs sector is CP-conserving at lowest order, $\mathrm{CP}$-violating effects can be induced by non-vanishing phases entering via potentially large higher-order contributions. As a consequence, all three neutral Higgs bosons $h, H$ and $A$ mix with each other, leading to the mass eigenstates $h_{1}, h_{2}, h_{3}$. The three mass eigenstates share their couplings to gauge bosons, i.e. the sum of the squares of the couplings of $h_{1}, h_{2}, h_{3}$ to gauge bosons is equal in good approximation to the squared coupling of the SM Higgs boson to gauge bosons. Depending on the mixing between the three Higgs bosons, the coupling of at least one of the three Higgs states to gauge bosons can be heavily suppressed compared to the SM case. Such a situation occurs in the CPX benchmark scenario of the MSSM [69], where over a large part of the parameter space, the lightest Higgs state, $h_{1}$, decouples from gauge bosons, while the second-lightest Higgs boson can have a large branching ratio into a pair of the lightest Higgs bosons, $h_{2} \rightarrow h_{1} h_{1}$.

In the LEP Higgs searches [12] carried out in the CPX scenario, an unexcluded parameter region remained for a mass of the lightest Higgs boson of about $45 \mathrm{GeV}$ and moderate $\tan \beta$. This is illustrated in Fig. 2.1, which shows the parameter regions excluded at 95\% C.L. by the topological cross section limits obtained at LEP [11,12] (as implemented in the program HiggsBounds [81]), making use of the currently most advanced theory prediction for Higgs boson cascade decays obtained in Ref. [82].

The case of a low Higgs mass in this scenario has only partially been explored at the LHC so far, and it seems difficult to close the "hole" shown in the LEP coverage of Fig. 2.1 with the standard search channels, see Refs. [70-72] for experimental studies of the ATLAS collaboration and Refs. $[83,84]$ for a discussion of other possible search channels for covering this parameter 


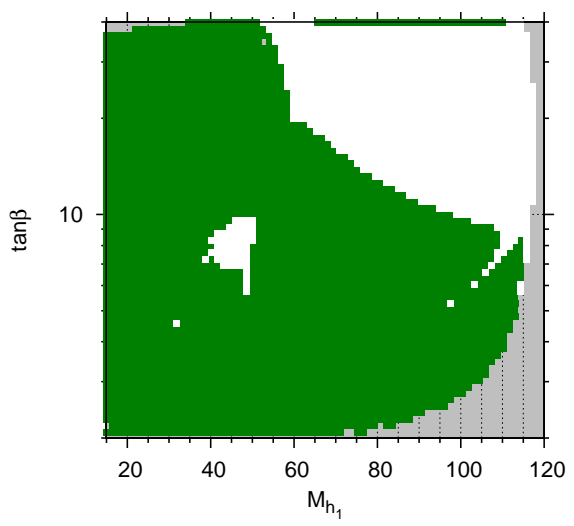

Fig. 2.1: Coverage of the LEP Higgs searches in the $\left(M_{h_{1}}, \tan \beta\right)$ plane of the CPX benchmark scenario of the MSSM. The plot (from Ref. [81]) shows the parameter regions excluded at 95\% C.L. (green) by the topological cross section limits obtained at LEP [11,12], using the theory prediction for Higgs cascade decays from Ref. [82].

region.

Thus, the described scenario of the MSSM with non-vanishing phases of the parameters could be a case where no Higgs signal appears in the first $10 \mathrm{fb}^{-1}$ at the LHC. On the other hand, in this scenario supersymmetric particles would be detected at the LHC with the first $10 \mathrm{fb}^{-1}$. One would therefore be in a situation where on the one hand there would be clear evidence for new physics, compatible with the discovery of supersymmetry. On the other hand, the experimental evidence for a light Higgs boson, which is required in this framework, would be lacking. This could be a strong case for an $e^{+} e^{-}$linear collider (LC) with a center-of-mass energy of at least $250 \mathrm{GeV}$ to explore whether or not the Higgs mechanism is realized in nature.

\subsubsection{A Higgs boson with SM-like $W W / Z Z$ couplings but unusual visible decays}

It is even possible that $\mathrm{LHC}_{10 / \mathrm{fb}}$ would not see a signal for a (possibly quite light, $m_{h} \sim 100 \mathrm{GeV}$ ) Higgs boson with SM-like $W W / Z Z$ couplings due to the fact that the Higgs decays to a pair of particles each of which subsequently pair-decays. Possibilities for the latter pairs include: $g g ; c \bar{c}$; $\chi_{2} \chi_{1}$ where $\chi_{1}$ is invisible and $\chi_{2}$ decays to $f \bar{f} \chi_{1}$; and to a less extent, $\tau^{+} \tau^{-}$. The first two cases arise in the NMSSM scenario explored in Refs. [73, 74]. In the NMSSM, even in the absence of $\mathrm{CP}$ violation there are three CP-even Higgs bosons, $h_{1,2,3}$, two CP-odd Higgs bosons, $a_{1,2}$ and a charged Higgs boson pair, $h^{ \pm}$. At moderate to high $\tan \beta(\tan \beta \gtrsim 3)$ the most attractive scenarios are such that the $h_{1}$ has quite SM-like couplings to $W W, Z Z, f \bar{f}$ but decays predominantly via $h_{1} \rightarrow a_{1} a_{1}$ which dominates over $h_{1} \rightarrow b \bar{b}$. In these scenarios, $a_{1}$ has a mass smaller than $2 m_{b}$ so that $a_{1}$ decays to $\tau^{+} \tau^{-}$or to a mixture of $g g, c c, \mu^{+} \mu^{-}$, depending on the precise value of $m_{a_{1}}$. Since such a scenario allows $m_{h_{1}}$ to be as low as $86 \mathrm{GeV}$, precision electroweak constraints are robustly satisfied and no fine-tuning is required to get the observed value of $M_{Z}$ since supersymmetric particle masses can be low if the Higgs boson with SM $W W, Z Z$ couplings has $m_{h_{1}} \lesssim 105 \mathrm{GeV}$.

As in the previous MSSM case, this kind of scenario predicts robust signals for supersym- 
metric particle production with $L=10 \mathrm{fb}^{-1}$ at the LHC associated with a lengthy wait for a Higgs boson signal. This does not imply that the Higgs boson could not eventually be discovered at the LHC. For example, a full study of the process $p p \rightarrow p h_{1} p$ (diffractive Higgs production) shows that $h_{1} \rightarrow a_{1} a_{1} \rightarrow \tau^{+} \tau^{-} \tau^{+} \tau^{-}$could be detected with $L \sim 100-300 \mathrm{fb}^{-1}$ [85]. Assuming that all trigger issues can be solved, the key to detecting this signal turns out to be track counting, in particular keeping only events with a small number of centrally produced tracks. The same is likely to apply to other possible observation modes such as the $W W$ fusion mode: $p p \rightarrow W W X \rightarrow h_{1} X \rightarrow \tau^{+} \tau^{-} \tau^{+} \tau^{-} X$. Of course, $e^{+} e^{-} \rightarrow Z h_{1}$ with $h_{1} \rightarrow a_{1} a_{1} \rightarrow \tau^{+} \tau^{-} \tau^{+} \tau^{-}$ would be highly visible at a linear collider.

At low $\tan \beta$ in the NMSSM, additional very natural scenarios arise with even more exotic features. In particular, one can have [86]: (i) strong mixing among the $h_{1,2,3}$, leading to suppressed $W W, Z Z$ couplings for all; (ii) dominant $h_{2} \rightarrow h_{1} h_{1}$ (as well as $h_{1,2} \rightarrow a_{1} a_{1}$ ) decays; and (iii) exotic $h^{+} \rightarrow W^{+} a_{1}$ decays. The $h_{1}, h_{2}$, and $h^{ \pm}$can all have mass $\lesssim 100 \mathrm{GeV}$ without conflicting with LEP and Tevatron limits. Adding CP violation to the model would lead to even more possibilities.

Future colliders might prove crucial to understanding the kinds of Higgs scenarios discussed above. At a linear collider, any Higgs boson with substantial $Z Z$ coupling will be produced via $e^{+} e^{-} \rightarrow Z h$ and detected as a peak in the $M_{X}$ spectrum of $e^{+} e^{-} \rightarrow Z X$ where the $Z$ decays to fully visible final states (e.g. $e^{+} e^{-}, \mu^{+} \mu^{-}, j j$ ). The various Higgs decay channels could then be studied. For example, the full $h_{1} \rightarrow a_{1} a_{1} \rightarrow \tau^{+} \tau^{-} \tau^{+} \tau^{-}$decay chain could be reconstructed. Detection of direct $a_{1}$ production (e.g. via $e^{+} e^{-} \rightarrow \nu \bar{\nu} a_{1} a_{1}$ ) would typically be quite challenging due to the low mass of the $a_{1}$ and its singlet nature. Both a $\gamma \gamma$ and a $\mu^{+} \mu^{-}$collider would also be excellent facilities for studying the $h_{1}$. The muon collider would be particularly interesting due to the fact that $\Gamma_{\text {tot }}^{h_{1}}$ is typically a factor of 10 larger than $\Gamma_{\text {tot }}^{h_{\mathrm{SM}}}$ due to the extra $h_{1} \rightarrow a_{1} a_{1}$ decays. As a result, the beam energy resolution required to scan the $h_{1}$ peak at the muon collider could be about a factor of 10 larger than that needed to scan the $h_{\mathrm{Sm}}$ peak. A direct scan of the $h_{1}$ peak would provide the most accurate measurement of $\Gamma_{t o t}^{h_{1}}$ and allow the highest precision measurements of all the couplings of the $h_{1}$ [87].

\subsubsection{Invisible Higgs decays}

As a third example of a possibly difficult scenario for Higgs searches at the LHC, we discuss the case where a Higgs boson decays predominantly into invisible particles. This can happen for instance in the MSSM via the decay of a Higgs boson into a pair of the lightest neutralino, in models with extra dimensions via the decay of a Higgs boson into KK neutrinos, in models with neutrinos of a 4 th generation, etc.

A priori a predominantly invisibly decaying Higgs boson does not automatically imply that the corresponding signature will not be detectable at the LHC. However, the Higgs search in such a scenario is expected to be more difficult than in the SM case, which could give rise to the fact that no Higgs boson signal can be established in the first $10 \mathrm{fb}^{-1}$ at the LHC.

In order to estimate the potential for invisible Higgs searches, a model independent variable, $\xi$, is commonly introduced to take into account possible modifications in the Higgs production 
cross-section as well as the invisible Higgs decay fraction:

$$
\xi^{2}=\frac{\sigma_{\mathrm{BSM}}}{\sigma_{\mathrm{SM}}} B R(H \rightarrow \text { inv. })
$$

The most promising processes (see, e.g., Ref. [88]) for the search of an invisible Higgs boson at the LHC are vector boson fusion $q q \rightarrow q q+\not_{\mathrm{T}}(\mathrm{VBF})$ and the production in association with a $Z$ decaying into two leptons $(Z H)$. Both analyses are confronted with substantial challenges arising from backgrounds due to pile-up, beam halo, cosmic muons, and from detector effects (instrumental noise, detector calibration). One of the most significant experimental challenges for the VBF analysis is to record enough events of interest while keeping the QCD background within the allowable trigger rate. This is particularly challenging since the VBF signature consists of two jets and large transverse missing energy which is copiously produced in a hadronic environment. In addition to the trigger and pileup effects which are hard to estimate at the moment, a control of the systematic uncertainties associated with jets and missing transverse energy will be essential to set physics limits with the VBF channel. Unlike the VBF analysis, the $Z H$ analysis relies on lepton triggers which are much cleaner and are not plagued by large QCD backgrounds. As such, the trigger is not expected to be a concern in this case. Various systematic uncertainties associated with the leptons, jets, and missing transverse energy appear to be significantly less important than for the VBF analysis.

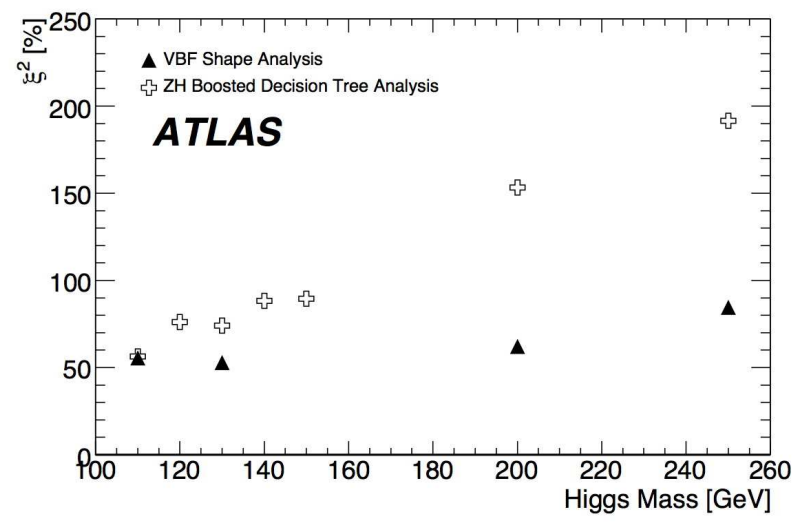

Fig. 2.2: Sensitivity at the $95 \%$ C.L. to an invisible Higgs boson with ATLAS for both the VBF and $Z H$ channels with $30 \mathrm{fb}^{-1}$ of data assuming only Standard Model backgrounds. The open crosses show the sensitivity for the $Z H$ analysis, and the solid triangles show the sensitivity for the VBF shape analysis. Both these results include systematic uncertainties. If we take the uncertainty on the signal efficiency into account, the sensitivity in $\xi_{95}^{2}$ could vary by up to $\pm 2.4 \%$ and $\pm 10.5 \%$ for the $Z H$ and VBF analyses, respectively (from Ref. [2]).

The current best estimate of the sensitivity of the ATLAS detector for observing an invisibly decaying Higgs boson is presented in Fig. 2.2. Naively scaling the ATLAS results discussed in Ref. [2], given for $30 \mathrm{fb}^{-1}$ in Fig. 2.2 to $500 \mathrm{fb}^{-1}$ and $10 \mathrm{fb}^{-1}$ of data yields the following results

\footnotetext{
${ }^{1}$ This is a naive estimate only since systematic errors do not scale with integrated luminosity.
} 
( $\xi_{95}^{2}$ denotes the $95 \%$ C.L. limit on $\left.\xi^{2}\right)$ :

\begin{tabular}{|c|c|c|}
\hline$m_{h}[\mathrm{GeV}]$ & $\begin{array}{c}\xi_{95}^{2} \text { for VBF } \\
10 / 30 / 500\left[\mathrm{fb}^{-1}\right]\end{array}$ & $\begin{array}{c}\xi_{95}^{2} \text { for } Z H \\
10 / 30 / 500\left[\mathrm{fb}^{-1}\right]\end{array}$ \\
\hline \hline 110 & $.95 / .55 / .14$ & $.99 / .57 / .18$ \\
\hline 130 & $.95 / .55 / .14$ & $1.28 / .74 / .23$ \\
\hline 200 & $1.05 / .6 / .15$ & $2.6 / 1.5 / .35$ \\
\hline
\end{tabular}

The results scaled to $500 \mathrm{fb}^{-1}$ suggest that the Higgs searches at the LHC could ultimately probe branching ratios at $\sim 15 \%$ for masses between $120-160 \mathrm{GeV}$. On the other hand, the prospects for $10 \mathrm{fb}^{-1}$ are much worse, illustrating the fact that a scenario with a sizable Higgs branching ratio into invisible particles can imply the absence of a Higgs signal at least in the early LHC data.

At an $e^{+} e^{-}$LC, the detection of invisible decays of a Higgs boson with SM-like $Z Z$ coupling that decays partly or entirely to invisible channels would be much easier owing to the cleaner experimental environment. The studies presented in Refs. [89] on the potential evidence for an invisible decay channel of the Higgs boson at a future $350 \mathrm{GeV} \mathrm{LC}$, in the process $Z(\rightarrow q q) H$, conclude that, with $500 \mathrm{fb}^{-1}$ of data, an invisible Higgs boson can be discovered down to branching ratios of $\sim 2 \%$ for masses between $120-160 \mathrm{GeV}$.

Once an invisible Higgs boson is discovered, the question of measuring its properties remains. If the invisible channel is dominant, the mass may nevertheless be accessible at the LHC through the measurement of the production rate. The $Z H$ production rate, steeply falling as a function of the mass, is sensitive to the Higgs mass. Moreover, the ratio of the production processes can also provide an independent estimate of the Higgs mass. Results from a parton level study [90] suggest that with $100 \mathrm{fb}^{-1}$ of data the mass can be determined to within 10-30 GeV. In contrast, the mass resolution at the ILC is expected to be significantly better using the recoil method. Studies suggest it should be in the tens of $\mathrm{MeV}[24,91]$.

Another mechanism for producing and detecting the invisible decay of a Higgs boson is via $p p \rightarrow p h p$. The mass $m_{h}$ can be accurately determined by observing the forward going protons and measuring the missing mass, in close analogy to what is possible at a linear collider. The existence of the sharp peak in the missing mass spectrum allows one to determine $m_{h}$ with an error of $\sim \pm 2 \mathrm{GeV}$ and dramatically reduces any background contributions. Second, observation of a Higgs boson in this way (i.e. via Pomeron-Pomeron fusion) implies that the produced boson must be neutral, colourless, flavourless and have natural parity, $P=(-1)^{J}$, with $J P=0^{+}$being by far the most likely. A preliminary study in Ref. [92] suggests there is reason to be optimistic that Higgs detection in this manner will be possible, but there are many issues related to triggering, backgrounds and pile-up that require a full study. In the end, event rates are likely to be low, but if it is known from other channels that an invisibly decaying Higgs boson is present, even a few clean events would have a dramatic impact on verifying the nature of the Higgs boson and determining its mass.

\subsubsection{Strategies for the future}

The situation of "no Higgs boson at the $\mathrm{LHC}_{10 / \mathrm{fb}}$ ", even if the Higgs mechanism is realized in nature, can result from two scenarios: 
(1) Reduced couplings and branching ratios can lead to a "later Higgs discovery" at the LHC.

(2) The structure of Higgs couplings and decays can lead to the situation where the Higgs boson permanently escapes detection at the LHC.

Due to the absence of a signal, it will not be possible to determine at the $\mathrm{LHC}_{10 / \mathrm{fb}}$ which scenario is realized, whereas the decision for a future collider/experiment can depend critically on the answer to that question. If several $\sim 2 \sigma$ effects compatible with a Higgs boson were measured, this could speak in favor of scenario (1). The absence of such effects, together with the absence of any hint for a scenario replacing the Higgs mechanism could speak in favor of scenario (2). The detection of SUSY particles would clearly speak in favor of the realization of either scenario (1) or (2). Any "solution" for scenario (2) would also yield a solution for scenario (1). Consequently, in the case of a completely unclear situation at the $\mathrm{LHC}_{10 / \mathrm{fb}}$ it would be sufficient to consider scenario (2) and its implications.

In the following we will briefly investigate what is specific about the two scenarios/models, and how each model can best be investigated in the future:

- Scenario (1): Here, a collider would be needed that provides sufficiently clean Higgs production modes with a high enough rate to compensate for reduced couplings. This could be the case of (i) possibly the sLHC with its very high luminosity if backgrounds and systematic uncertainties can be brought sufficiently well under control or (ii) an $e^{+} e^{-}$LC such as the ILC or CLIC running at high luminosity. The latter will have the advantage of providing a clean environment allowing to detect small branching ratios and a detection will be possible despite other (invisible) decay modes. It has been shown that rare decay modes such as to $c \bar{c}$ and to $\mu^{+} \mu^{-}$can be detected when running at high luminosity. A clean environment would also be helpful to detect experimentally difficult decay patterns, such as the case of a Higgs boson decaying to other light bosons, where the latter would decay into rather soft jets (possibly $c \bar{c}$ ) or $\tau$ leptons.

- Scenario (2): Here, a collider would be needed that provides a variety of Higgs production modes (for instance radiation off top- and/or bottom-quarks or other new particles, such as scalar tops in the case of SUSY) with a sufficiently high rate and controllable backgrounds and that is capable to detect any kind of unusual decay patterns. If the Higgs mechanism is responsible for generating the masses of the weak gauge bosons $Z$ and $W^{ \pm}$, one would expect that at least one Higgs boson should have a significant coupling to the weak bosons (unless the coupling to gauge bosons is shared among a large number of Higgs bosons, see e.g. Ref. [80]). The experimental capabilities for probing production processes where the Higgs boson couples to gauge bosons are therefore of particular importance. The experimental environment needed to probe this scenario could be provided by (i) an $e^{+} e^{-}$ LC. The particular power of the LC is its ability to look for $e^{+} e^{-} \rightarrow Z H$ in the inclusive $e^{+} e^{-} \rightarrow Z X$ missing-mass, $M_{X}$, distribution recoiling against the $Z$ boson. Even if the Higgs boson decays completely invisibly or different Higgs signals overlap in a complicated way, the recoil mass distribution will reveal the Higgs boson mass spectrum of the model. At a LC a Higgs boson could furthermore be produced in weak-boson fusion, in association with heavy fermions, $e^{+} e^{-} \rightarrow f \bar{f} H$, with $f \bar{f}=t \bar{t}, b \bar{b}$, or sfermions, $\tilde{t} \overline{\tilde{t}}, \tilde{b} \overline{\tilde{b}}$, in the case of SUSY. Having potentially the couplings to up- and down-type (s)fermions at hand strongly reduces the possibility of a complete suppression; (ii) a muon collider, where the Higgs 
boson could be produced by its couplings to muons directly in the $s$-channel. However, a reduction of the Higgs couplings to all down-type fermions or to muons in particular can be possible; (iii) a high-energy $e^{+} e^{-}$or $\gamma \gamma$ collider with a large reach for heavy Higgs bosons that are outside the reach of the LHC.

\subsection{Higgsless/technicolor scenarios}

A general feature of Higgsless models ${ }^{2}$ is the appearance of new spin-1 states that replace the Higgs boson such that perturbative unitarity of $W W \rightarrow W W$ scattering can be achieved up to a few TeV. These states are usually the lightest non-standard particles. While replacing the Higgs boson in maintaining perturbative unitarity is a relatively easy goal in principle, the construction of explicit Higgsless models yielding a satisfactory description of EW precision tests is a much harder task to achieve. In technicolor models, the SM Higgs sector is replaced with new well defined strongly coupled four dimensional dynamics within a renormalizable gauge theory ${ }^{3}$.

\subsubsection{Walking technicolor models}

Extended technicolor allows for fermion masses, but to avoid FCNCs, the scale of extended technicolor would be too high and these fermion masses would be too small unless technicolor models rely on walking dynamics, i.e., the idea that the technicolor coupling remains large and nearly constant over a wide range of energy scales. The use of fermions in higher dimensional representations or the admixture of different matter representations achieve near conformal dynamics for a small number of flavors [93].

Using novel methods to analyze the non-perturbative dynamics of strongly coupled gauge theories, it has been possible [94] to find a large number of underlying gauge theories which can be employed to break the electroweak symmetry dynamically while alleviating problems associated with potentially dangerous flavour changing neutral currents as well as the tension with precision electroweak data. The phenomenology of these models is analyzed using the low energy effective theory developed in Ref. [95]. The basic ingredients are the presence of vector and axial-vector spin-one resonances, their coupling with the SM fields and the presence of a scalar resonance/composite Higgs boson. To reduce the parameter space it is convenient to make use of the modified Weinberg sum rules (WSR) for walking dynamics [96] and impose, at the effective Lagrangian level, known constraints. The LHC signatures are scanned in terms of the mass of the axial resonance $m_{A}$, which gets linked with the vector one via the modified WSRs, and the coupling $\tilde{g}$ which controls both the overall strength of the heavy spin-one and spin-zero interactions as well as the mixing with the SM gauge fields. The larger $\tilde{g}$ is, the less the heavy spin-one states mix with the SM gauge bosons. Walking theories with fermions in higher dimensional representations have also been explored in Ref. [97].

\footnotetext{
${ }^{2}$ By a "higgsless" model we mean here an effective model where no explicit fundamental Higgs boson is introduced. A composite Higgs boson emerging from a strongly interacting sector can still be present in the low energy spectrum.

${ }^{3}$ Many models of strong EW symmetry breaking predict the appearance of scalar resonances with masses comparable or even lighter than those of vector resonances. These scalar resonances could actually play an important role in the unitarization process.
} 
In another class of walking technicolor models (see, e.g., Ref. [98]), a near conformal, $\beta\left(\alpha_{T}\right) \sim 0$, coupling is achieved by having a lot of technimatter. The Technicolor Strawman Model $[99,100]$ has often been used as a reference for Tevatron analyses and for phenomenological studies at the LHC. As all (electroweak charged) technimatter contributes to the weak scale, the more matter, the lower the fundamental strong interaction scale becomes. Consequently, these models predict light spin-one resonances, around $700 \mathrm{GeV}$ or even less. The best discovery channels for these resonances are via their decays into pairs of SM gauge bosons. Due to the large chiral symmetry group present in these models, there are typically several new pseudoscalar states (technipions) as well.

A general classification of possible four dimensional gauge theories which can have a near conformal (walking) behavior appeared in Ref. [94]. Several new models of walking technicolor dynamics were introduced there as well. In these models there is no systematic calculation of the precision EW parameters. However, the matter content and beta function are very different from QCD, so estimates of these precision EW parameters based on rescaling the QCD values should not be trusted.

\subsubsection{Drell-Yan production of heavy vectors}

Heavy spin-one resonances can be produced at the LHC through the DY processes $p p \rightarrow R_{1,2}$ where $R_{1,2}$ are the physical eigenstates which take into account the mixing with the SM gauge bosons. To estimate the LHC reach for DY production of the $R_{1,2}^{0}$ and $R_{1,2}^{ \pm}$resonances, the following final state lepton processes were considered (see Ref. [95] for details):

(a) $l^{+} l^{-}$signature from the process $p p \rightarrow R_{1,2}^{0} \rightarrow l^{+} l^{-}$,

(b) $3 l+\mathbb{E}_{T}$ signature from the process $p p \rightarrow R_{1,2}^{ \pm} \rightarrow Z W^{ \pm} \rightarrow 3 l \nu$.

Acceptance cuts $\left(\left|\eta^{l}\right|<2.5\right.$ and $\left.p_{T}^{l}>15 \mathrm{GeV}\right)$ on the leptons are applied and an additional cut on the missing transverse energy is imposed $\left(E_{T}>15 \mathrm{GeV}\right)$. For process (a), the dilepton invariant mass distribution $M_{l l}$ is used to separate the signal from the background, while for process (b), the analysis relies on the transverse mass variable $M_{3 l}^{T}$. For $\tilde{g}=2$, clear signals from the leptonic decays of the resonances are seen up to masses of around $2 \mathrm{TeV}$ with only $10 \mathrm{fb}^{-1}, \sqrt{s}=10 \mathrm{TeV}$. Both peaks from $R_{1,2}^{0}$ may be resolved. For larger values of $\tilde{g}$, this signal deteriorates and will only be observable for small vector masses. Fortunately, for large $\tilde{g}$, the triple-vector coupling is enhanced and a signal in the $M_{3 l}^{T}$ distribution may be observed at large masses as presented in Fig. 2.3. A few events could potentially be observed with only $10 \mathrm{fb}^{-1}$ at $\sqrt{s}=14 \mathrm{TeV}$ in this channel.

\subsubsection{Associate production of a composite Higgs boson and a SM vector boson}

When the strong dynamics produces a light scalar resonance with the quantum numbers of the Higgs boson (composite Higgs boson), the resonant production of heavy vectors can enhance significantly the $\mathrm{WH}$ and $\mathrm{ZH}$ production compared to the SM. Figure 2.4 presents event-rate plots for $p p \rightarrow W^{ \pm} H \rightarrow W^{ \pm} Z Z \rightarrow 4 l+2 j$ for $\sqrt{s}=10 \mathrm{TeV}$ (resp. $14 \mathrm{TeV}$ ) and $10 \mathrm{fb}^{-1}$ (resp. $100 \mathrm{fb}^{-1}$ ) of integrated luminosity. The peaks correspond to the contribution from a heavy axial spin-one state with mass $m_{A}=0.5$ and $1 \mathrm{TeV}$. A similar analysis can be performed for the $p p \rightarrow Z H$ channel. It is possible to discover vectors (the mostly axial technicolor spin-one state) with masses 

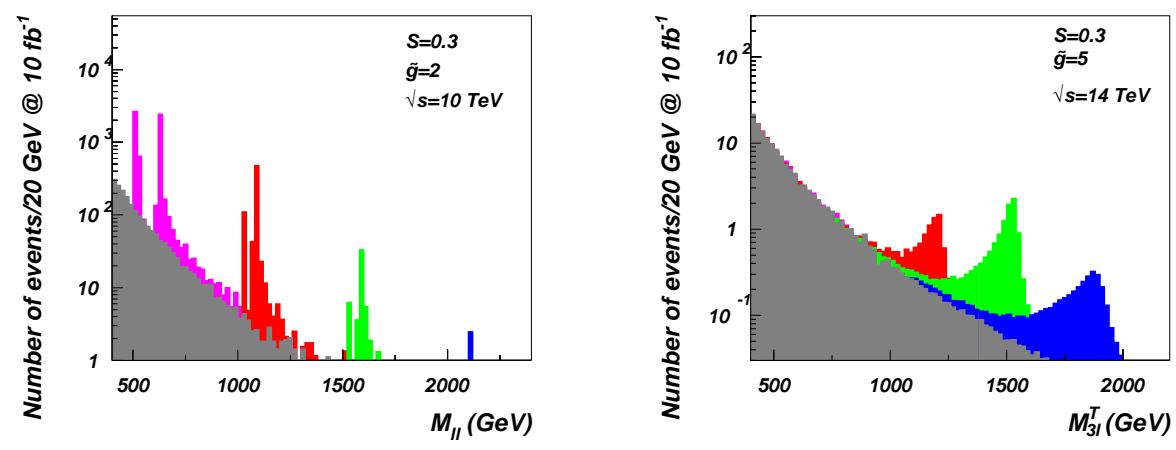

Fig. 2.3: Invariant and transverse mass distributions for signal and background processes (a) and (b) of Sect. 2.3.1.1. The model parameters considered are $\tilde{g}=2$ and $\tilde{g}=5$ in the left and right figures respectively (note also the different energy for the two figures) and $m_{A}=0.5 \mathrm{TeV}$ (purple), $1 \mathrm{TeV}$ (red), $1.5 \mathrm{TeV}$ (green) and $2 \mathrm{TeV}$ (blue). The background appears in grey. In both cases, the $S$ parameter [101] is equal to 0.3.

up to $1 \mathrm{TeV}$ while the signal worsens when increasing the mass of the composite Higgs boson. With $10 \mathrm{fb}^{-1}$ only, the LHC could be able to observe the interplay of a composite Higgs boson and the mostly axial vector boson only if both are very light. Thus, the scenario of a composite Higgs boson could lead to the observation of a Higgs-like signal at the LHC but could also give rise to a situation where no clear signal can be established with the first $10 \mathrm{fb}^{-1}$ at the LHC.
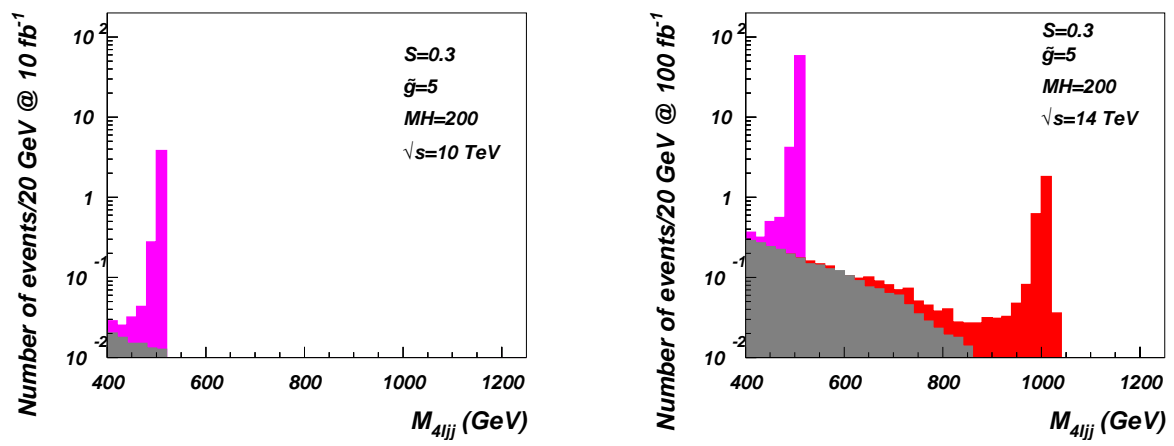

Fig. 2.4: $p p \rightarrow W^{ \pm} H \rightarrow W^{ \pm} Z Z \rightarrow 4 l+2 j$ with $\sqrt{s}=10 \mathrm{TeV}, 10 \mathrm{fb}^{-1}$ and $14 \mathrm{TeV}, 100 \mathrm{fb}^{-1}$ on the left and right respectively with $m_{A}=0.5 \mathrm{TeV}$ (purple), $1 \mathrm{TeV}$ (red). The parameters of the model correspond to an intrinsic $S$ parameter [101] equal to 0.3 and a heavy vector dimensionless coupling $\tilde{g}=5$.

\subsubsection{Effective lagrangian approach to resonances}

The phenomenology of heavy vectors at high-energy colliders, as well as their role in EW precision tests (EWPT), has been widely discussed in the literature recently. However, most of the existing analyses are based on specific dynamical assumptions, such as considering these vector states as the massive gauge bosons of a hidden local symmetry. As recently discussed in Ref. [102], these assumptions may be too restrictive for generic models with strong dynamics at the TeV scale, 
and going beyond these assumptions, the sole exchange of heavy vectors can satisfy EWPT. More generally, the construction of an appropriate effective theory including only SM fields and these new light states is a very efficient tool to discuss theoretical and phenomenological constraints on such states.

The effective theory proposed in Ref. [102] is based on the following rather general assumptions:

- The new strong dynamics is invariant under a global chiral symmetry $G=S U(2)_{L} \times$ $S U(2)_{R}$, broken spontaneously into $H=S U(2)_{L+R}$ (the custodial symmetry of the SM Higgs potential), and under a discrete parity symmetry $\left(P: S U(2)_{L} \leftrightarrow S U(2)_{R}\right)$.

- A pair of vector $(V)$ and axial-vector $(A)$ states, belonging to the adjoint representation of $H$, are the only new light dynamical degrees of freedom below a cut-off scale $\Lambda \sim(2 \div 3) \mathrm{TeV}$.

Under these assumptions the dynamics of the new spin-1 states is controlled by three effective couplings: $G_{V}, F_{V}$ and $F_{A}$, expected to be of $\mathcal{O}(v \approx 250 \mathrm{GeV})$ by naïve dimensional analysis, and the masses of the two new states $\left(M_{V}\right.$ and $\left.M_{A}\right)$. The coupling $G_{V}$ controls the effective coupling of the new vector states to the Goldstone bosons of the theory (or the longitudinal components of SM gauge bosons), while $F_{V}$ and $F_{A}$ control the (gauge-invariant) mixing of the new states with the transverse components of the SM gauge bosons.

By construction, $G_{V}$ is rather constrained after the unitarity condition is imposed. However, as shown in Fig. 2.5, the unitarity constraint alone does not pose a significant constraint on $M_{V}$. A more constrained picture is obtained if it is requires that the sole exchange of the two spin-1 states leads to a satisfactory description of EWPT. Under this stronger assumption it turns out that at least the vector state must be relatively light [102].

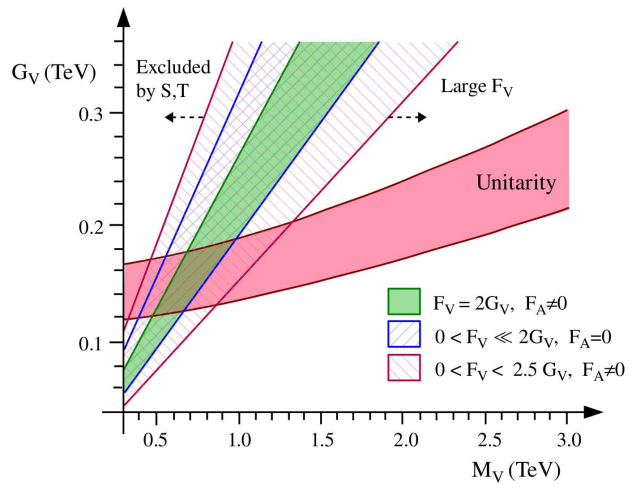

Fig. 2.5: Summary of unitarity and EWPT constraints (at 95\% C.L.) in the $\left(M_{V}, G_{V}\right)$ plane. From Ref. [102].

The free parameters of this effective theory are crucial ingredients for determining the possible signatures at future colliders. If only the unitarity constraint is used (assuming that other states play a significant role in EWPT), the lack of information on the spectrum does not allow one to draw firm conclusions about the detection of any of the new states at the LHC (see also Refs. $[103,104])$. In particular, the detection of a resonance of mass above $1 \mathrm{TeV}$ in $W W \rightarrow W W$ scattering will be extremely hard, even with high luminosity. An illustration of the typical signal expected in this process is shown in Fig. 2.6. Since the appearance of these resonances in 
$W W \rightarrow W W$ scattering is the most general property of Higgsless models, if at the LHC no new light state (including the Higgs boson) is found, the natural option to consider is a collider where $W W \rightarrow W W$ scattering can be probed to higher energies, unless there are hints that a fundamental Higgs boson could have been missed in the LHC searches.

If the mass of at least one of such states is relatively low, as suggested by the hypothesis that the sole exchange of such fields is relevant in EWPT, then it would be interesting to try to determine all the parameters of the effective theory. In such a case, Drell-Yan production of the new states, and the subsequent decay into $\ell^{+} \ell^{-}, W Z, W W$ or states with three SM gauge fields ( $W W W, W W Z, W Z Z$ ) are the most interesting final states to be studied. For sufficiently light masses (and relatively large couplings), some of these signals could be within the LHC reach (see, e.g., Fig. 2.6).
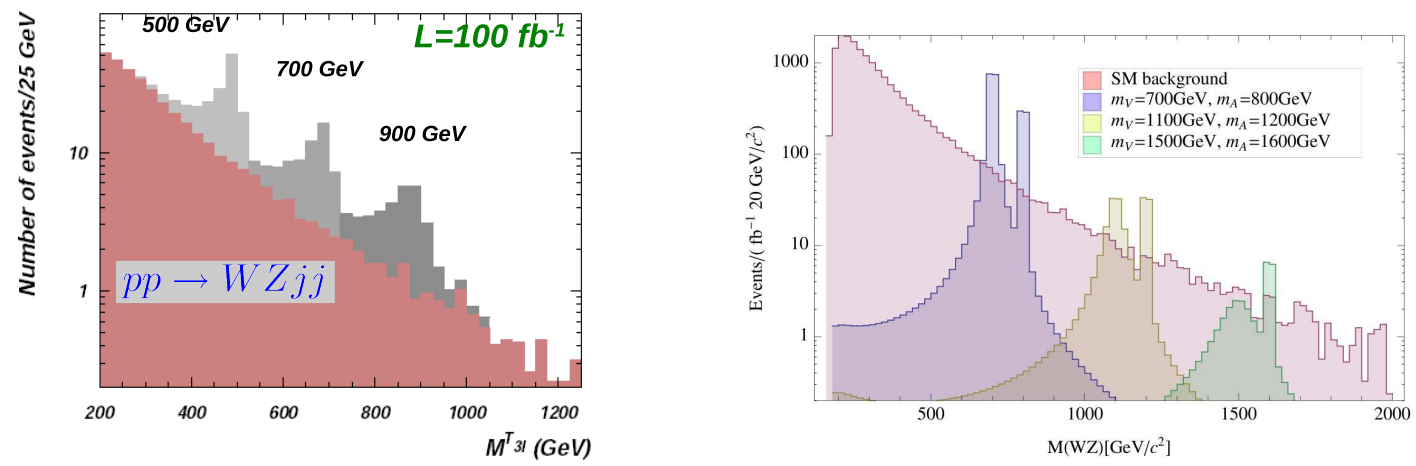

Fig. 2.6: Left: Signal events in $p p \rightarrow W Z+2$ jets (from $W W$ fusion) with leptonic decays of both $W$ and $Z$ bosons and standard $W W$-fusion cuts on the two jets. From Ref. [105]. Right: Invariant mass spectrum of $W Z$ pairs produced in $p p \rightarrow W Z$ at $\sqrt{s}=14 \mathrm{TeV}$, with contributions from $V$ and $A$ states. All resonance signals have been obtained assuming $F_{A}=F_{V}=2 G_{V}$ (condition that maximises the signal). The SM background corresponds only to the irreducible electroweak production of $W Z$ pairs. The plot does not include the decay branching ratios of $W$ and $Z$ bosons, as well as any experimental cut. From Ref. [106].

\subsubsection{Extra dimensional models}

To make predictions of the LHC signatures of viable technicolor (TC) models, we can: (i) set up 4D models with field content which we anticipate gives walking behavior and extract whatever information we can, or (ii) consider the subset of near-conformal TC models with a weakly coupled (and therefore calculable) 5D description. With simplest Anti-de Sitter setup, the agreement with EW precision constraints is still problematic. In the absence of any symmetry to enforce a small $S$ oblique parameter, the only option is a tuned cancellation. Currently there are two techniques for achieving such a cancellation: Cured Higgsless, and Holographic Technicolor. In each case, the cancellations required for $S$ lead to distinct signatures at the LHC. 


\subsubsection{Cured Higgsless (CHL)}

The matching of 5D to $4 \mathrm{D}$, which sets parameters involved in the $\mathrm{EW}$ precision observables, is dependent on the fermion wavefunction localization along the extra dimension. By tuning the fermions to have very specific profiles, the EW observables can be brought back to an acceptable range. This cancellation happens when the fermion profiles are nearly flat. Though the couplings between the SM fermions and the KK excitation of the $W$ and the $Z$ are small $(\sim 0.1 \times \mathrm{SM})$, they are still important at the LHC and Drell-Yan production is usually a dominant channel with the following properties [106, 107]:

- Charged $W_{K K}$ appear in $W Z(3 \ell+\nu)$ final states.

- $Z_{K K}$ and its higher tiers can most easily be seen in dilepton channels.

- Unlike low scale technicolor, there is no $W \gamma$ mode.

- There are actually two $Z_{K K}$ modes - the KK partners of the $Z$ and $\gamma$. These states are nearly degenerate, $\Delta M<\Gamma_{Z_{K K}}$, which may lead to interesting interference effects [108].

- Minimal bulk $S U(2)_{L} \otimes S U(2)_{R}$ symmetry implies there are no technipions in the spectrum.

Since tuning the fermion wavefunction is required to satisfy EWPT, the observation of KK fermions and measurements of their couplings are important. These KK fermions should have similar mass to the KK gauge bosons, and can be either singly or doubly produced at colliders. One interesting possibility is the production of $q_{K K} q_{K K}$ (rather than $q_{K K} \overline{q_{K K}}$ ) through $t$-channel KK boson exchange which, following the decay $q_{K K} \rightarrow q^{\prime} W^{ \pm}$, can lead to final states with two same sign leptons.

\subsubsection{Holographic Technicolor (HTC)}

A second option for cancelling contributions to the $S$ parameter is to drop the assumption that all fields feel the same metric. Specifically we can allow the vector and axial combinations of the $S U(2)$ to feel different warp factors with the following expected consequences $[109,110]$ :

- by divorcing vector from axial, we can dial the individual warp factors such that we have nearly degenerate $\left(m_{V} \sim m_{A}\right)$ or even inverted $\left(m_{A}<m_{V}\right)$ spectra, reducing $S$ without having to change the fermions.

- different warp factors can be recast as local operators and to achieve $S \sim 0$ the coefficients of these local operators must be much larger than naïve dimensional analysis (NDA) estimates. Thus the HTC scenario is also tuned [111].

- the signature of HTC is the presence of nearly degenerate KK gauge bosons, both charged and neutral. Therefore we should observe: (i) two charged resonances in $W Z(3 \ell+\nu)$, (ii) two neutral resonances in dileptons and (iii) a possible nonzero coupling between a photon, a SM $W$ and a charged resonance.

\subsubsection{Deconstructed/BESS models}

The discretization of the compact fifth dimension of the previous models to a lattice generates the so-called deconstructed theories which are chiral Lagrangians with a number of replicas of the gauge group equal to the number of lattice sites. The delocalization of fermions along the 
fifth dimension is equivalent, in the deconstructed picture, to direct couplings between new vector bosons and SM fermions [112]. In the simplest version of this latter class of models, corresponding to just three lattice sites and gauge symmetry $S U(2)_{L} \times S U(2) \times U(1)_{Y}$ (the so-called BESS model [113]), the requirement of a small $S$ oblique parameter implies that the new triplet of vector bosons is almost fermiophobic. Then the only production channels for their search are those driven by boson-boson couplings. However, the minimal three-site model can be extended by inserting an additional lattice site. This four-site Higgsless model, based on the $S U(2)_{L} \times S U(2)_{1} \times S U(2)_{2} \times$ $U(1)_{Y}$ gauge symmetry, predicts two neutral and four charged extra gauge bosons, $Z_{1,2}$ and $W_{1,2}^{ \pm}$, and satisfies the EWPT constraints without necessarily having fermiophobic resonances [114]. Within this framework, the more promising Drell-Yan processes become particularly relevant for the extra gauge boson search at the LHC. Clearly a future Linear Collider operating in the TeV range has indirect sensitivity to the four-site model and can profile the low mass $Z_{1}$ and $Z_{2}$.

\subsubsection{Drell-Yan production of heavy vectors at the LHC}

The four-site Higgsless model predicts six new gauge bosons $Z_{1,2}$ and $W_{1,2}^{ \pm}$which can be produced at the LHC through Drell-Yan channels. There are two interesting classes of processes:

(a) $p p \rightarrow Z_{1,2} \rightarrow l^{+} l^{-}$characterized by two isolated charged leptons in the final state,

(b) $p p \rightarrow W_{1,2}^{ \pm} \rightarrow l \nu_{l}$ giving rise to one isolated charged lepton plus missing energy.

A study for the LHC of these channels is described in Ref. [114]. In Fig. 2.7, the distributions both in the charged and neutral Drell-Yan channels are shown. Here standard acceptance cuts $\left(p_{T}^{l}>20 \mathrm{GeV},\left|\eta_{l}\right|<2.5\right)$ are applied. Two particular sets of free parameters describing the four-site Higgsless model are used with two resonances at $1 \mathrm{TeV}$ and 1.25 TeV. The full Drell-Yan process, considering signal and SM-background, is computed at the EW and QCD leading order.

For two different sets of fermionic couplings $\left(b_{1}, b_{2}\right)$ shown, for example, the signal strength can vary significantly. With the first set, the new gauge bosons could be discovered already at the LHC start-up, with a minimum integrated luminosity of $1 \mathrm{fb}^{-1}$ while with the second set high luminosity will be required.

\subsubsection{Four-site model at a future linear collider}

One of the most striking manifestations of new physics at a TeV-class $e^{+} e^{-} \mathrm{LC}$ will come from the sudden increase of the $e^{+} e^{-} \rightarrow f \bar{f}$ cross section indicating the $s$-channel production of one or more new particles. The existence of the two neutral gauge bosons, $Z_{1,2}$, of the four-site model and their properties can be precisely studied, if their mass is lower than the LC centre-of-mass energy. Figure 2.8 shows two examples of $Z_{1,2}$ scanning, namely $M_{1,2}=(680,850) \mathrm{GeV}$ at a $1 \mathrm{TeV}-\mathrm{LC}$ and $M_{1,2}=(1600,2000) \mathrm{GeV}$ at a $3 \mathrm{TeV}-\mathrm{LC}$ with a luminosity of $100 \mathrm{fb}^{-1}$. For each scenario, two sets of fermionic couplings inside the region allowed by the EWPT were chosen and significant signals are observed. In this preliminary analysis the initial state radiation is taken into account but no beamstrahlung is included.

Even beyond the kinematical reach for $s$-channel production, a TeV-LC could prove the existence of new vector resonances up to scales of several TeV by studying the electroweak observables, e.g., $\sigma_{\mu \mu}, \sigma_{b \bar{b}}, A_{F B}^{\mu}, A_{F B}^{b}$, and indirect bounds from a $1 \mathrm{TeV}-\mathrm{LC}$ can exclude a portion of the parameter space left open by the LHC, as shown in Fig. 2.9 where the region excluded by a 

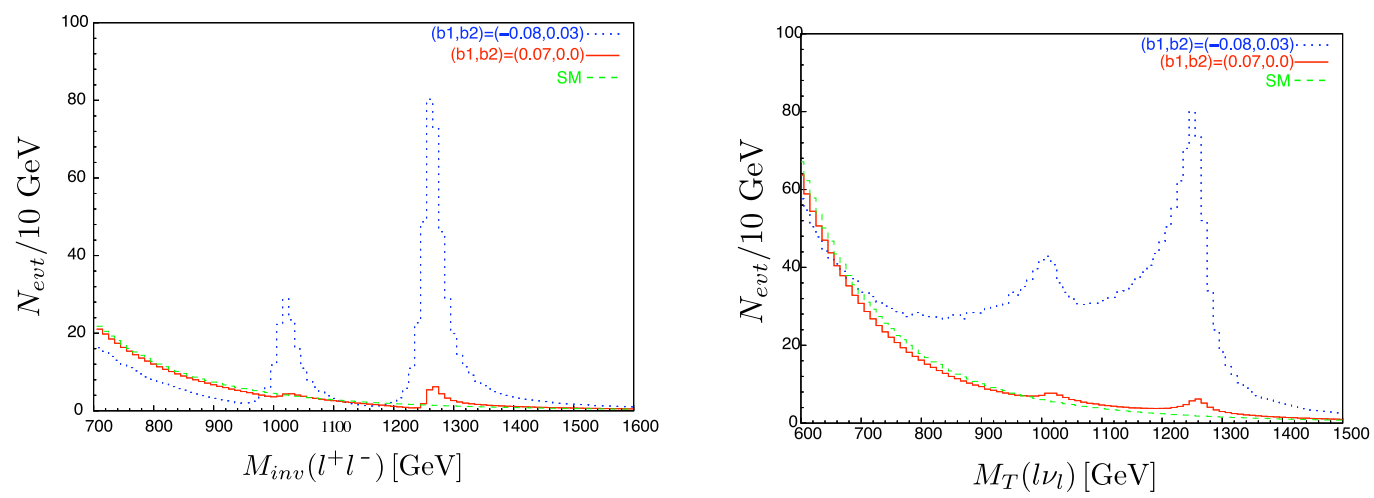

Fig. 2.7: Total number of events per $10 \mathrm{GeV}$ versus the dilepton invariant mass $M_{l^{+} l^{-}}$for the process $p p \rightarrow l^{+} l^{-}$(left) and versus the lepton transverse mass, $M_{T}\left(l \nu_{l}\right)$ (right), for the process $p p \rightarrow l \nu_{l}$ with an integrated luminosity of $10 \mathrm{fb}^{-1}$ at the LHC with $\sqrt{s}=14 \mathrm{TeV}$ for $M_{1,2}=(1000,1250) \mathrm{GeV}$ and the two sets of parameters $\left(b_{1}, b_{2}\right)$ corresponding to different couplings to fermions. The $e, \mu$ and charge conjugate channels have been summed. (From Ref. [114]).
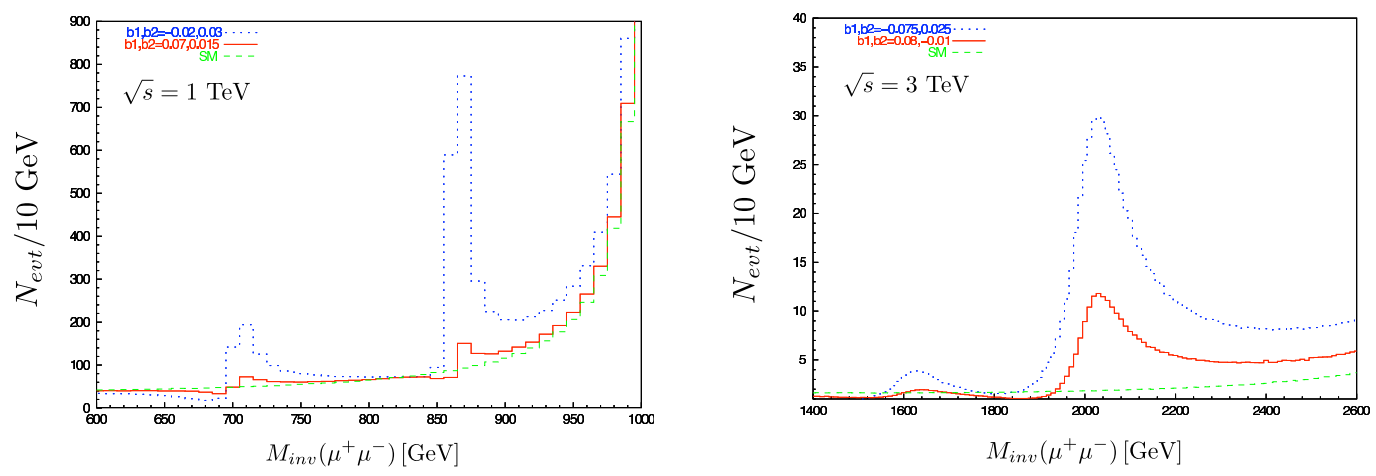

Fig. 2.8: Total number of events per $10 \mathrm{GeV}$ versus the dimuon invariant mass, $M_{\mu^{+}} \mu^{-}$for the process $e^{+} e^{-} \rightarrow \mu^{+} \mu^{-}$at a $1 \mathrm{TeV}$ (left), $3 \mathrm{TeV}$ (right) $e^{+} e^{-}$Linear Collider with a $100 \mathrm{fb}^{-1}$ integrated luminosity, for $M_{1,2}=(680,850) \mathrm{GeV}$ (left) and $M_{1,2}=(1600,2000) \mathrm{GeV}$ (right) and for two sets of fermionic couplings as quoted in the figures. Initial state raditation is included, beamstrahlung is not taken into account. (From Ref. [115]).

$1 \mathrm{TeV}$-LC is obtained by comparing the deviations of the four-site model predictions from the SM ones, with the uncertainties, assuming that they are statistically dominated. The relative statistical accuracies are rescaled from those obtained for a total luminosity of $1 \mathrm{ab}^{-1}$ accumulated at CLIC running at $\sqrt{s}=3 \mathrm{TeV}$, including the effect of $\gamma \gamma \rightarrow$ hadrons background [116]. Better sensitivity could be obtained by considering polarized beams.

\subsubsection{WW Scattering at ATLAS/CMS}

Most of the processes described in the earlier sections rely on the leptonic final states, which provide relatively clean samples, with signal resonances expected to peak over backgrounds that can be estimated from sidebands with relative ease. 


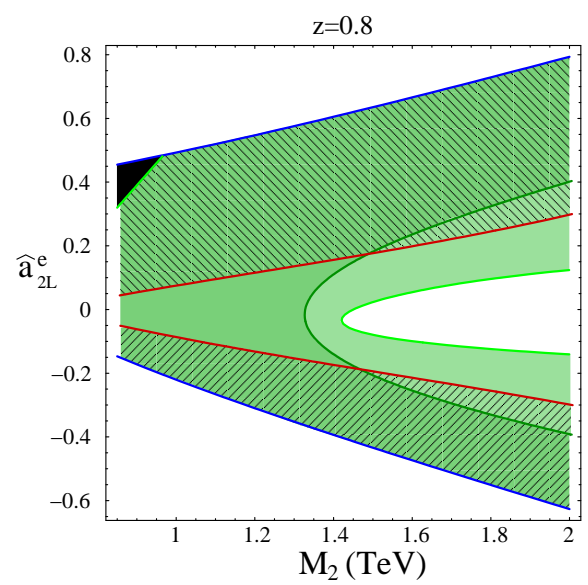

Fig. 2.9: $3 \sigma$-exclusion plots in the plane $\left(\hat{a}_{2 L}^{e}, M_{2}\right)$ for $M_{1} / M_{2}=0.8\left(\hat{a}_{2 L}^{e}\right.$ is the left-handed coupling between the $Z_{2}$-boson and the SM electron in units of the electric charge, $M_{2}$ is the mass of the $Z_{2}$-boson). The upper and lower parts are excluded by EWPT, the black triangle is the region excluded by the direct search at the Tevatron for a luminosity of $4 \mathrm{fb}^{-1}$. The dashed region is excluded by the Drell-Yan processes at the $\mathrm{LHC}$ at $\mathrm{L}=100 \mathrm{fb}^{-1}$. The green (light-green) region is excluded by a $1 \mathrm{TeV}-\mathrm{LC}$ by combining the measurements of $\sigma_{\mu \mu}, \sigma_{b \bar{b}}, A_{F B}^{\mu}, A_{F B}^{b}$ for a luminosity of $10 \mathrm{fb}^{-1}\left(1 \mathrm{ab}^{-1}\right)$. (From Ref. [115]).

On the other hand, the LHC experiments will focus not only on the leptonic final states, but also on hadronic decays of the vector bosons (VB). For example, particularly for integrated luminosities of up to $100 \mathrm{fb}^{-1}$, both ATLAS and CMS analyses for VB scattering in semi-leptonic final states, in which one of the VBs decays hadronically, appear to be as or more promising than fully leptonic final states for observing signals from possible new resonances (Higgs boson or other) at the $0.5-1 \mathrm{TeV}$ range.

However, there are two major challenges to the reconstruction of semi-leptonic final states. The first one is the significantly higher background, from sources like $t \bar{t}+\mathrm{jets}$ and $\mathrm{VB}+\mathrm{jets}$. In the particular case of VB scattering, additional requirements can be put on the event topology: the presence of two high-energy forward jets (tag jets), resulting from the quarks that radiated the scattering VBs, is one of the well-known characteristic feature of this process. The electroweak nature of the interaction, with no color exchange in the rapidity interval between the tag jets, further provides a handle against backgrounds from QCD processes with central jet activity. Studies from both collaborations indicate that they can be used under pile-up expected for $10^{33} \mathrm{~cm}^{-2} \mathrm{~s}^{-1}$ luminosity [2,117].

The second challenge is in the reconstruction of the hadronic VBs themselves, particularly when they are highly-boosted $\left(p_{T} \gtrsim 250 \mathrm{GeV}\right)$, which is common in Higgless models. The decay products are often collimated and reconstuction from a pair of jets is no longer applicable. In such cases, it is possible to reconstruct the VB candidates as single massive jets and perform substructure analysis to suppress QCD backgrounds. The feasibility of such an approach was previously shown at a hadron-level study of $W W$ scattering [118]. Recently, the ATLAS Collaboration has performed this study using both fast and full detector simulation [2].

Besides complementing the inclusive searches, the VB fusion signature can be exploited to study the VB scattering as a probe of electroweak symmetry breaking, by investigating the $M_{V V}$ 

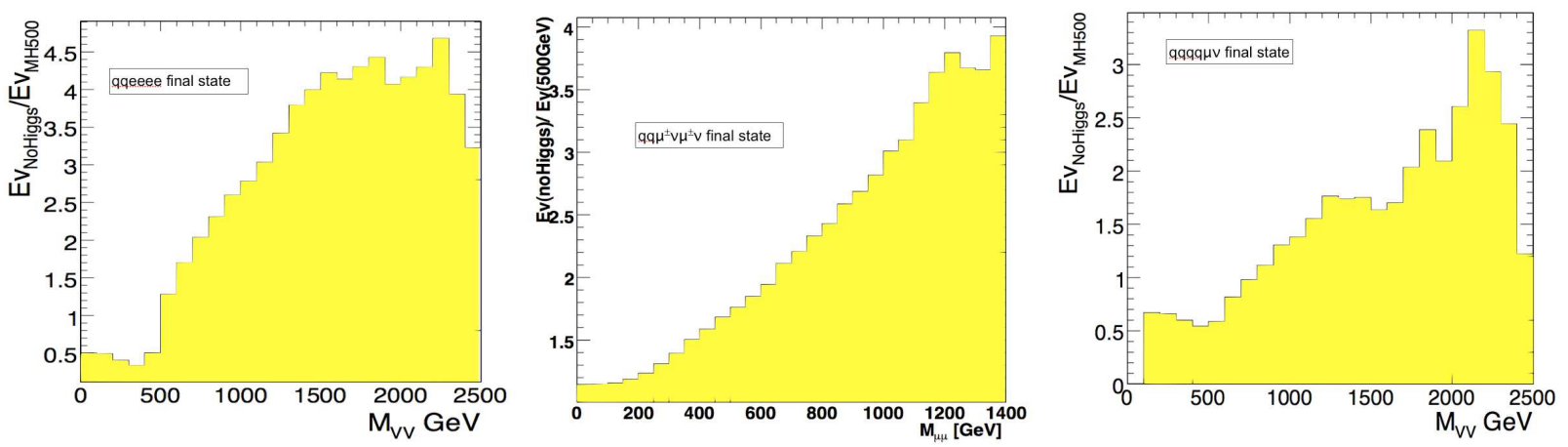

Fig. 2.10: Higgs/no-Higgs boson signal discriminant as a function of the minimum $M_{V V}$ considered, for different final states, in the CMS detector [117].

spectrum in case of Higgs presence (where a peak is expected) and absence (where the shape at high masses changes due to the different contributions of longitudinal and transverse couplings of the VBs) [119]. A feasibility study in the CMS detector, based on $\mathcal{O}\left(\alpha_{E W}^{6}\right)+\mathcal{O}\left(\alpha_{S}^{2}\right)$ six-fermionfinal-state generator Phantom [120], shows the separation potential at reconstruction level, after $60 \mathrm{fb}^{-1}$ of integrated luminosity while running at $10^{33} \mathrm{~cm}^{-2} \mathrm{~s}^{-1}$, with the corresponding pile-up taken into account. Figure 2.10 shows the value of the discriminant used $\left(\int_{M_{\text {cut }}}^{\infty} d M_{V V} \frac{d \sigma_{\text {noHiggs }}}{d M_{V V}}\right.$ / $\int_{M_{c u t}}^{\infty} d M_{V V} \frac{d \sigma_{m_{H}=500 \mathrm{GeV}}}{d M_{V V}}$ ), applied to the simulated signal samples, as a function of $M_{c u t}$, for three different final states. At a high luminosity collider, this discriminant has a potential to yield information on the unitarization mechanism.

Likewise, recent parton-level studies show the Higgs/no Higgs separation potential in realistic conditions, taking into account the contributions from irreducible backgrounds, with a simple cut-based analysis developed for a higher integrated luminosity [121].

Measurements of multi-boson final states are expected to start with the extraction of Tevatroncompetitive limits for anomalous triple-gauge couplings in the first few hundred $\mathrm{pb}^{-1}$ of data. They will be followed first by searches for model-dependent states (like techni-resonances at $\mathcal{O} \sim$ $10 \mathrm{fb}^{-1}$ ) and later by generic searches for resonances (mass up to $\sim 1 \mathrm{TeV}$ at $\mathcal{O} \sim 50-200 \mathrm{fb}^{-1}$ ). Discoveries of much heavier resonances, their spin analyses and the extraction of a detailed spectrum up to 2-3 TeV will probably need few hundreds of $\mathrm{fb}^{-1}$ and will benefit from the sLHC and other future colliders.

\subsubsection{EW chiral lagrangian in absence of resonance}

If there exists no new particle below 2-3 TeV, the scattering of $W, Z$ gauge bosons is well described in terms of the EW chiral lagrangian [122]. Assuming custodial symmetry, there are only two coefficients contributing to the scattering amplitudes at order $p^{4}$ :

$$
\mathcal{A}\left(W_{L}^{a} W_{L}^{b} \rightarrow W_{L}^{c} W_{L}^{d}\right)=\mathcal{A}(s, t, u) \delta^{a b} \delta^{c d}+\mathcal{A}(t, s, u) \delta^{a c} \delta^{b d}+\mathcal{A}(u, t, s) \delta^{a d} \delta^{b c}
$$

with [123]

$$
\mathcal{A}(s, t, u)=\frac{s}{v^{2}}+\frac{4}{v^{4}}\left(2 \alpha_{5} s^{2}+\alpha_{4}\left(t^{2}+u^{2}\right)\right)+\frac{1}{16 \pi^{2} v^{4}}\left\{-\frac{1}{12}\left(3 t^{2}+u^{2}-s^{2}\right) \log \left(\frac{-t}{\mu^{2}}\right)\right.
$$




$$
\left.-\frac{1}{12}\left(3 u^{2}+t^{2}-s^{2}\right) \log \left(\frac{-u}{\mu^{2}}\right)-\frac{s^{2}}{2} \log \left(\frac{-s}{\mu^{2}}\right)\right\} .
$$

Figure 2.11 shows the region of parameter space where perturbative unitarity breakdown is postponed from 1.2 TeV to $2 \mathrm{TeV}$ (above $2.1 \mathrm{TeV}$, there is no value of $\alpha_{4,5}$ that unitarizes all the scattering channels simultaneously). The sensitivity of the ILC to these parameters in the channels $e^{+} e^{-} \rightarrow W^{+} W^{-} \nu \nu$ and $e^{+} e^{-} \rightarrow Z Z \nu \nu$ at $\sqrt{s}=800 \mathrm{GeV}$ [124] is somewhat better than what can be achieved at the LHC $[125,126]$.

To avoid unitarity violation, it is common [127] to invoke a unitarization procedure such as the Padé or the K-matrix scheme. The latter case leads to a non-resonant enhancement of the cross section, with respect to a low-mass Higgs scenario, of longitudinal vector boson scattering. This will be extremely difficult to observe at the LHC. The Padé unitarization scheme, which gives a good description of meson scattering, leads to the presence of resonances [127]. At the LHC, it will require a few tens of $\mathrm{fb}^{-1}$ to observe such resonances for masses up to $\sim 1.2 \mathrm{TeV}[2]$.

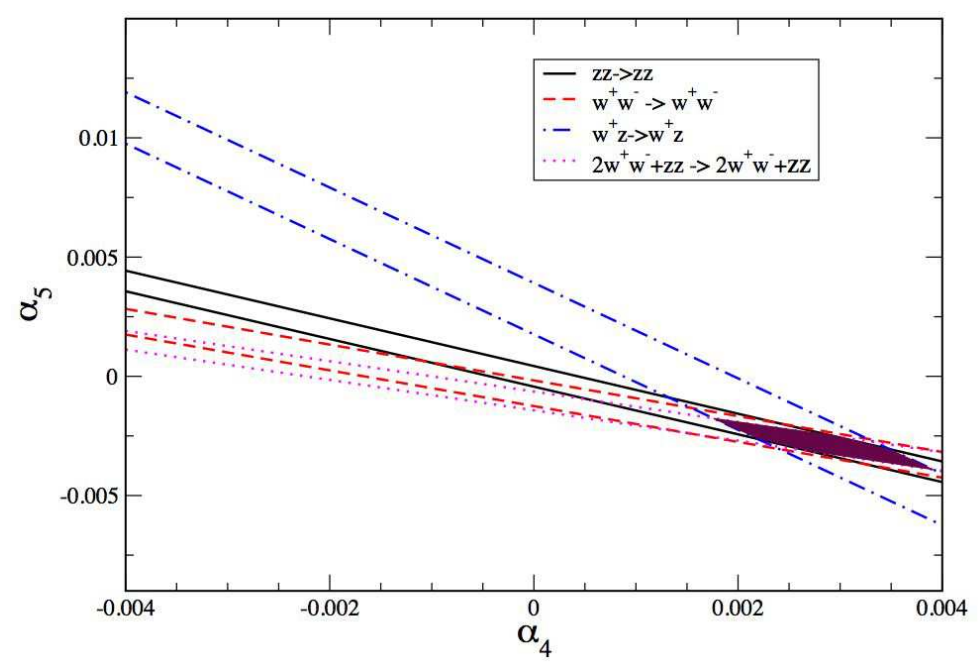

Fig. 2.11: Allowed values of $\alpha_{4,5}$, see eq. (2.7), for perturbative unitarity to hold at $\sqrt{s}=2 \mathrm{TeV}$. The region between the red dashed lines shows the allowed values of $\alpha_{4,5}$ to hold for the scattering process $W^{+} W^{-} \rightarrow W^{+} W^{-}$. Similarly, black, blue, and pink lines delineate the bounds for the scattering $Z Z \rightarrow$ $Z Z, W^{+} Z \rightarrow W^{+} Z$ and $\left(2 W^{+} W^{-}+Z Z\right) \rightarrow\left(2 W^{+} W^{-}+Z Z\right)$ respectively. This covers all the elastic scattering processes for the gauge bosons (the unitarity condition for $W^{ \pm} W^{ \pm} \rightarrow W^{ \pm} W^{ \pm}$is already satisfied if it holds for $W^{ \pm} Z \rightarrow W^{ \pm} Z$ ). At $\sqrt{s}=2.1 \mathrm{TeV}$, the different regions do not overlap anymore.

\subsection{Summary of WG2}

In the case where no clear Higgs-like signal will have been established with the first $10 \mathrm{fb}^{-1}$ of (understood) data at the LHC, one will be faced with the question whether one or more Higgs bosons exist but have been missed in the LHC searches because of their non-standard properties or whether there really is no fundamental Higgs boson, meaning that other new degrees of freedom or new dynamics beyond the Standard Model have to be present to achieve electroweak symmetry breaking while maintaining unitarity at high energy. 
The strategy for the future in such a scenario will clearly be influenced by the other phenomenology observed at the LHC. If other new physics is detected that seems to hint towards the realisation of (at least one) fundamental Higgs state in nature, such as the production of supersymmetric particles, and/or the gauge sector does not show indications of strong electroweak symmetry breaking dynamics, then this could be a strong case for an $e^{+} e^{-}$LC to explore the expected mass range for the Higgs boson and to precisely determine the nature of the other observed new physics. A particular strength of an $e^{+} e^{-}$LC would be to identify a Higgs boson produced in association with a $Z$ boson completely independently of its (possibly very unusual) decay properties by solely relying on the mass distribution recoiling against the $Z$ boson. If the Higgs mechanism is responsible for generating the masses of the weak gauge bosons, one would expect that at least one Higgs boson should have a significant coupling to the weak bosons so that it could be observable in this channel. An $e^{+} e^{-}$LC would possibly provide further Higgs production modes, for instance in association with heavy fermions or (in the case of SUSY) their scalar superpartners, and the measurements would allow one to determine the profile of a detected Higgs boson with high precision.

The sLHC could provide access to rare Higgs production and decay modes and with a sufficient amount of accumulated luminosity could possibly establish a Higgs signal. It could also profit from its enlarged mass reach for heavy Higgs bosons. A muon collider, in addition to production modes possible also at $e^{+} e^{-}$linear colliders, could also provide Higgs production in the s-channel. However, for motivating this option it would certainly be helpful if the Higgs coupling to muons had already been established independently at another collider.

If resonances or other indications of strong electroweak symmetry breaking dynamics are observed in gauge boson scattering, the strategy for the future appears to be less clear. An $e^{+} e^{-}$ $\mathrm{LC}$ operating in the $\mathrm{TeV}$ range would have good prospects to either directly produce resonances or indirectly probe the effects of the new dynamics. 


\section{WG3: Missing Energy}

B. Gripaios, F. Moortgat, G. Moortgat-Pick, G. Polesello (convenors)

P. Bechtle, K. Desch, B. Foster, V. Morton-Thurtle, K. Rolbiecki, J. Smillie, J. Tattersall, P. Wienemann

In this section the prospects for missing energy signals from new physics are discussed, both at the LHC and at a future linear collider. We also discuss potential synergy effects between both colliders. We summarize discovery potential, as well as methods for measuring masses, spins and other properties of new states. We also include searches for Dark Matter candidates at the different colliders.

\subsection{Introduction and scenarios}

Just before the actual start of the major experiment at the high-energy frontier, the LHC, is a particularly interesting time especially for new physics searches. Many physics models beyond the the Standard Model (SM) predict new sources for missing energy, therefore the main focus in this section is the discovery and the first analysis of new physics sectors. One of the favoured models for physics beyond the Standard Model is Supersymmetry (SUSY). It nicely overcomes major shortcomings of the SM, giving rise to unification of the coupling constants of the electroweak and strong interactions, a natural explanation of the hierarchy problem, and a suitable dark matter candidate. We therefore take SUSY as a representative new physics model, explain the respective physics potential of future colliders and apply the proposed experimental methods for analysing the properties of new particles within the SUSY context.

After giving an up-to-date discussion of the status of commisioning of missing transverse energy in the ATLAS and CMS detectors in Section 3.2, we discuss in Section 3.3 the discovery potential. Then, in Section 3.4, we describe methods for determining some properties of the new physics models, including accurate measurement of masses and spins. In Section 3.5, we include possible input for, as well as constraints from, dark matter experiments and outline the precision that is required to provide reasonable predictions.

We close the discussion in Section 3.6 with the inclusion of foreseen next high-energy options, namely the linear collider, and embed a comprehensive study of the ILC physics. In particular, we are interested in synergy effects between lepton- and hadron-collider types and discuss which outcomes from LHC results may have direct input on the current design efforts of the ILC.

\subsection{Commissioning of Missing Transverse Energy in the ATLAS and CMS experiments}

Neutrinos and other hypothetical weakly interacting particles pass through a collider experiment without detection. However, the presence of such particles in a collision can be inferred from the imbalance of the event's total momentum. This imbalance in a plane perpendicular to the beam direction is called Missing Transverse Momentum. Its magnitude is referred to as Missing Transverse Energy (MET). MET plays a principal role in studying SM physics as well as in searches for 
physics beyond the SM (e.g., Lightest Supersymmetric Particles (LSPs)). The traditional method for missing-transverse-momentum determination at hadron colliders is based on the calorimeter information.

In CMS, it is calculated as the negative vector sum of the transverse energies deposited in the calorimeter towers (above a noise threshold). This sum is corrected for (i) the presence of identified muons; and (ii) the under-measurement of the hadronic energy in the calorimeters, as explained in [128]. First, identified muons are corrected for by replacing the minimum ionizing transverse energy expected in the calorimeters by the transverse momentum of the associated track reconstructed in the central tracker. Second, the transverse energies of the reconstructed jets are replaced by those of the jet-energy-scale corrected jets. The sequential application of the muon and the jet-energy-scale corrections defines the current standard missing transverse energy in CMS (called CaloMET).

For the ATLAS experiment the baseline algorithm [2] starts from the energy deposits in calorimeter cells that survive a noise suppression procedure. Two noise suppression methods have been studied, one based on only using cells with energies larger than a threshold, the other based on only using cells in 3-dimensional topological calorimeter clusters [2,129]. The cells are then calibrated using global calibration weights depending on their energy density, and the MET calculated as the negative vector sum of the transverse energies deposited in the considered cells. Corrections are applied for the muon energy and for the energy lost in the cryostats. Only good quality muons in the muon spectrometer with a matching track in the internal tracker are considered, and the muon momentum as measured in the muon spectrometer is taken. The energy lost in the cryostats of the Liquid Argon (LArg) calorimeter is estimated using the correlation of energies between the last layer of the LArg calorimeter and the first layer of the hadronic calorimeter. In a subsequent step, the calibration of the calorimetric term is refined by recalibrating cells according to the reconstructed high- $P_{T}$ object they are assigned to. An important performance figure is the linearity, defined by the expression

$$
\text { Linearity } \equiv(\operatorname{MET}(\text { True })-\text { MET) } / \text { MET(True) }
$$

where MET(True) is the true value and MET is the measured value. The linearity for $A \rightarrow \tau \tau$ (where $A$ denotes a CP-odd Higgs bosons) with $M_{A}=800 \mathrm{GeV}$ is shown in Fig. 3.1 as a function of MET(True) for the different reconstruction steps described above. The bias of linearity at low values is due to the finite resolution of the measurement. The reconstructed MET is positive by definition, so the linearity is negative when the true MET is near to zero. For MET(True) > $40 \mathrm{GeV}$, the linearity is within $2 \%$.

The resolution of the measurement is estimated from the width of the distribution of the difference between true and estimated values of the MET $x$ and $y$ components in bins of the total transverse energy deposited in the calorimeters $\left(\Sigma E_{T}\right)$. The MET resolution, is shown in Fig. 3.2 as a function of $\Sigma E_{\mathrm{T}}$ for different ranges of $\Sigma E_{T}$, based on the study of different Monte Carlo samples. The dependence can be fitted with a function $\sigma=a \cdot \sqrt{\Sigma E_{T}}$ for values of $\Sigma E_{T}$ between 20 and $2000 \mathrm{GeV}$. The parameter $a$, which quantifies the MET resolution, varies between 0.53 and 0.57. Additional details on the ATLAS MET performance can be found in Ref. [2]

The resolution of the purely calorimetric MET measurement is significantly better in ATLAS than in CMS thanks to the better energy resolution and longitudinal energy containment of the ATLAS hadronic calorimetry. 


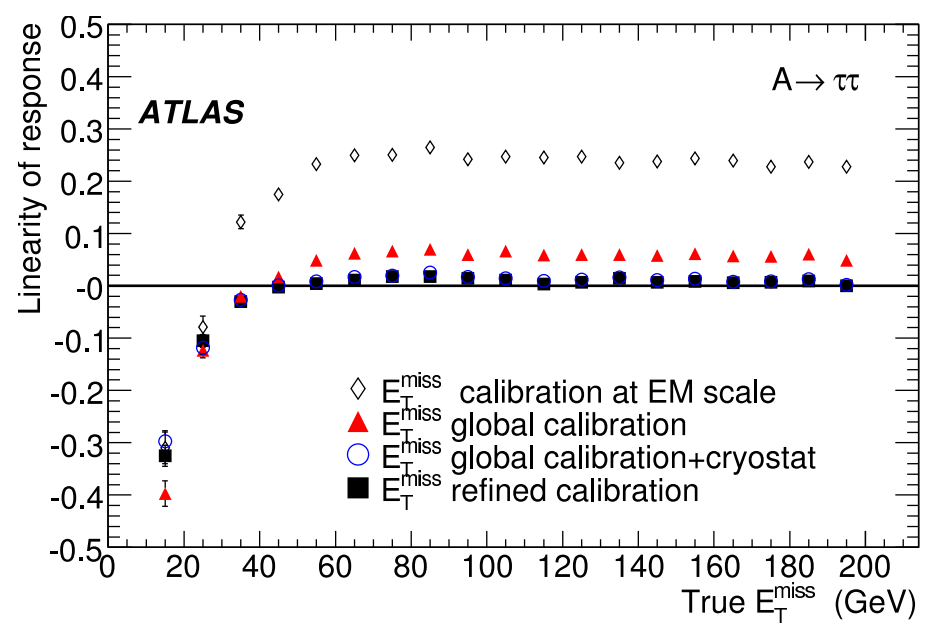

Fig. 3.1: Linearity of response for reconstructed MET as a function of the average true MET for $A \rightarrow \tau \tau$ events with $M_{A}=800 \mathrm{GeV}$ (taken from Ref. [2], see inside for details).
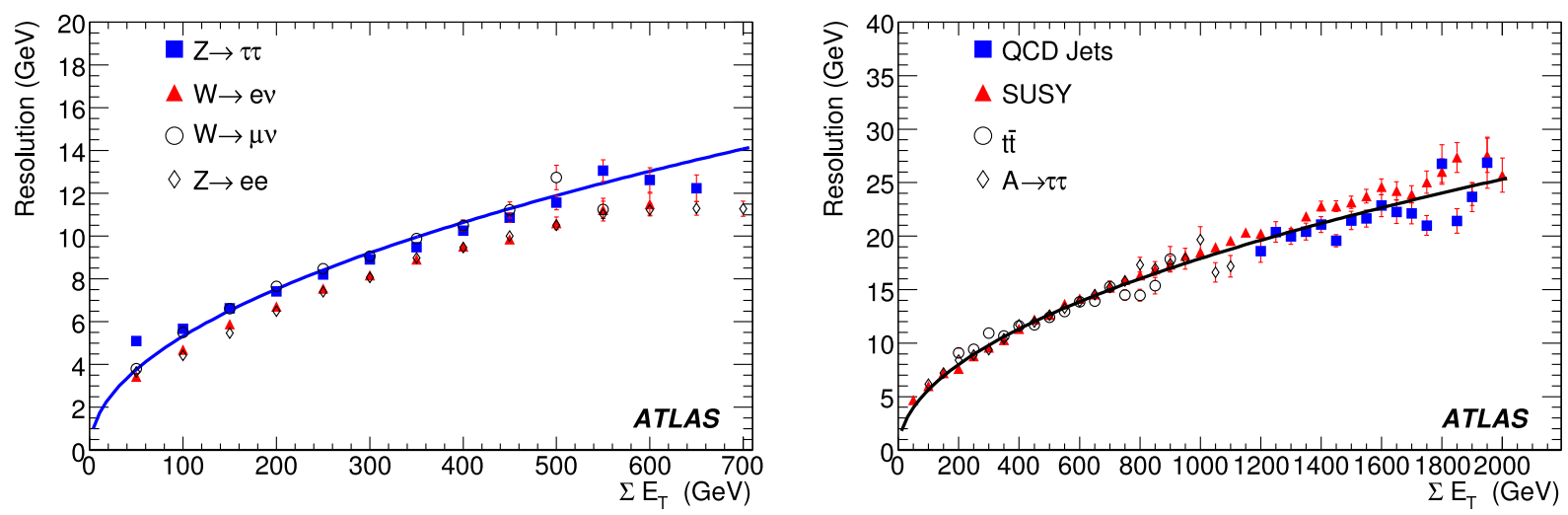

Fig. 3.2: Resolution of the two MET components with refined calibration as a function of the total transverse energy, $\Sigma E_{\mathrm{T}}$ for low to medium values (left) and for higher values (right). The curves correspond to the best fits of $\sigma=0.53 \sqrt{\Sigma E_{\mathrm{T}}}$ through the points from $Z \rightarrow \tau \tau$ events (left) and $\sigma=0.57 \sqrt{\Sigma E_{\mathrm{T}}}$ through the points from $A \rightarrow \tau \tau$ events (right). The points from $A \rightarrow \tau \tau$ events are for masses $M_{A}$ ranging from 150 to $800 \mathrm{GeV}$ and the points from QCD jets correspond to dijet events with $p_{T}$ between 560 and $1120 \mathrm{GeV}$. (Both plots are taken from Ref. [2], see inside for details.)

To cope with this issue, recently, two new methods for improving the purely calorimeterbased MET have been developed, exploiting information from the other subdetectors in CMS. In the Track-Corrected MET [130], the correction for the under-measurement of the hadronic energy in the calorimeters is replaced by a charged-particle-track-based correction: the transverse momentum of each reconstructed charged particle track is added to the total missing transverse momentum, from which the corresponding transverse energy expected to be deposited in the calorimeters is subtracted. The aforementioned muon correction is applied in turn. The resulting track-corrected missing transverse energy is shown to have a slightly better MET resolution and a reduced MET fake rate with respect to the calorimeter-only MET. Fig. 3.3 shows the improvement: the left plot 
illustrates that in $Z \rightarrow \ell \ell$ events, the track-corrected MET algorithm reduces the number of events with MET > 30 (50) GeV by a factor of 3.4 (6.8), compared to calorimetric MET corrected for muons only. The reduction is a factor of 3.0 (4.3) when compared to calorimetric MET corrected for both muons and the jet energy scale. Fig. 3.3 (right) illustrates that in $t \bar{t} \rightarrow \ell \ell+X$ events the accuracy of determining the MET resolution by track-corrected MET is improved by more than $25 \%(20 \%)$ compared to calorimetric MET corrected for muons only (muons + jet energy scale).
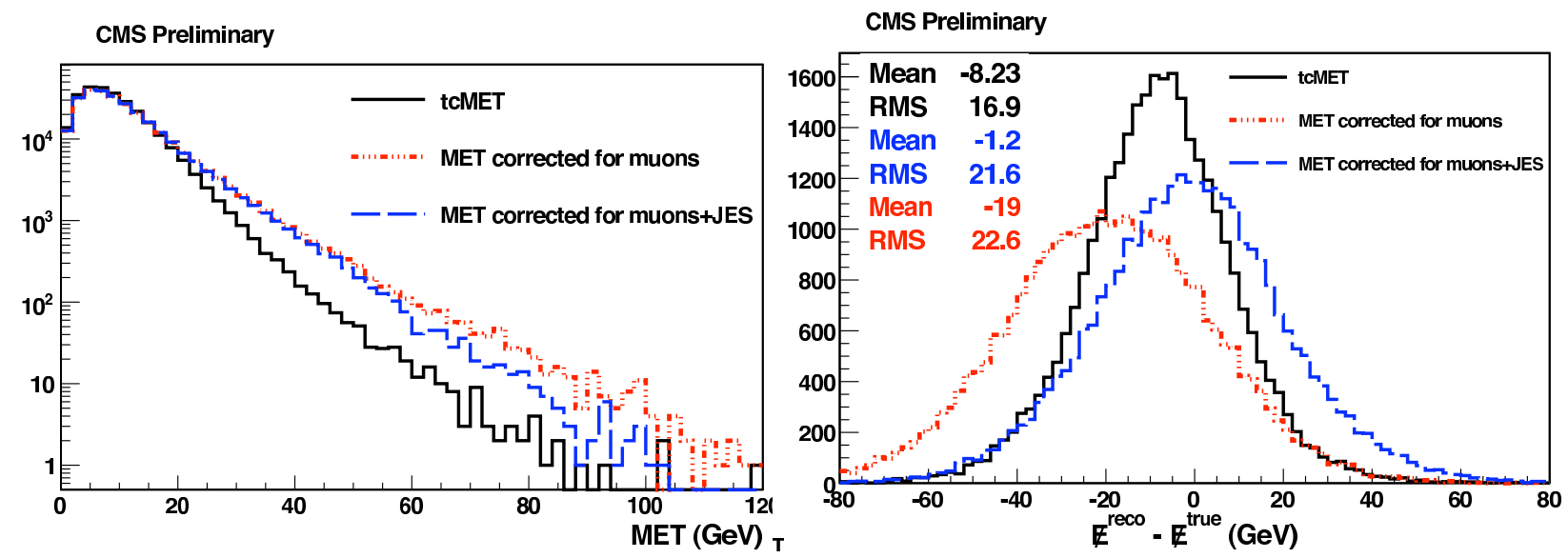

Fig. 3.3: Left Distributions for track-corrected MET (tcMET) and calorimeter-only MET in $Z \rightarrow \ell \ell$ events with the CMS detector. Right Distributions of $\Delta E_{T}^{\text {miss }}=\mathbb{E}_{T}^{\text {reco }}-\mathbb{E}_{T}^{\text {true }}$ for $t \bar{t} \rightarrow \ell \ell+X$ events with $\mathbb{E}_{T}^{\text {true }}>$ $50 \mathrm{GeV}$ (taken from [130]) .

A different approach is followed in the particle-flow event reconstruction: individual particles are reconstructed and identified by exploiting the characteristics of all CMS sub-detectors, towards efficiency and purity, and by using the built-in redundancies of the energy and direction measurements in the different sub-detectors, towards precision and accuracy. The Particle-Flow MET is determined as the modulus of the negative vector sum of the reconstructed-particle transverse momenta, with no need for posterior corrections. A detailed description of the algorithm, a report of its performance, and a number of related systematic studies can be found in [131]. Fig. 3.4 shows that the Particle Flow technique improves the MET resolution with almost a factor of two with respect to the calorimetric determination, irrespective of the true missing transverse energy.

The conceptual differences between the three approaches, from the simplest (CaloMET) to the most comprehensive (Particle-Flow MET), is expected to be a great asset when the first collision data are produced in the LHC. Independently of the respective performance, the largely independent systematic uncertainties and the undoubtedly different failure modes of the three methods will pave the road towards a rapid understanding and a robust determination of the missing transverse energy in CMS.

The MET commissioning activities in both experiments can be divided in three stages: (i) the pre-collision phase (no beam and single circulating beam period), where the detector can record cosmic muons and beam halo muons; (ii) the $10 \mathrm{pb}^{-1}$ phase, where the first proton-proton collisions will be registered, mainly minimum bias and QCD processes; and (iii) the $100 \mathrm{pb}^{-1}$ (and above) phase, after which sufficient statistics for physics processes such as $Z+$ jets or $t \bar{t}$ will be 


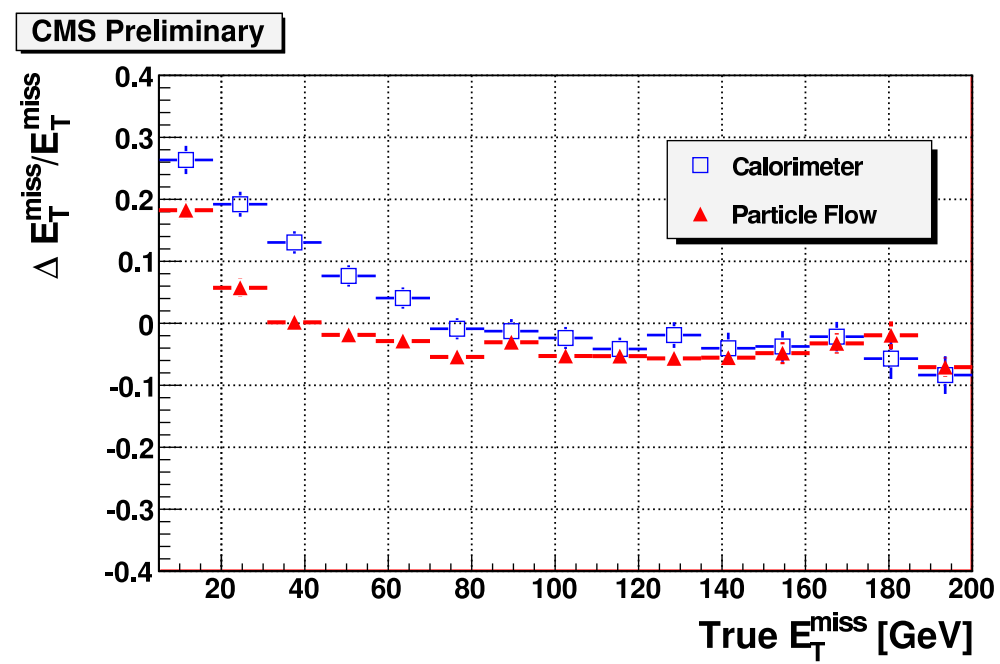

Fig. 3.4: Distribution of $\left(E_{\mathrm{T}, \text { reco }}^{\text {miss }}-E_{\mathrm{T}, \text { true }}^{\text {miss }}\right) / E_{\mathrm{T} \text {,true }}^{\text {miss }}$ as a function of the $E_{\mathrm{T} \text {,true }}^{\text {miss }}$, in a fully inclusive $\mathrm{t} \overline{\mathrm{t}}$ event sample, for particle-flow reconstruction (solid triangles) and for calorimeter-only reconstruction (open squares) in CMS (taken from [131]).

collected. In the pre-collision phase, many important instrumental procedures for constructing the missing transverse energy can already be commissioned. In particular, the handling of abnormal calorimeter cells (hot or dead) and the removal of detector noise can be and have been tested in the extensive campaigns of cosmics runs of the two detectors in 2009 with complete detector configuration. The MET Data Quality Monitoring (DQM) system can also be commissioned during this period. Procedures for the identification and removal of cosmic muons in future collision events can be verified. During the single circulating beam phase (i.e. when the LHC beams are being commissioned), the filters for beam halo muons can be tested and improved.

In the second phase, after the collection of the first collision data, the large cross section of QCD di-jet events will quickly allow the relative calibration of the calorimeters in order to obtain a uniform response over $\phi$ and $\eta$. Also the absolute calibration of the calorimeter cells, using single isolated tracks, will be performed. The jet energy scale corrections, needed for the corrections of calorimetric MET, will be derived using photon+jet and $Z+$ jet balance techniques.

In the third phase, when sufficient events of "standard candle" processes such as $W$ and $Z$ + jets or $t \bar{t}$ will have been recorded, the missing transverse energy can be validated using these control processes. The MET scale can be determined in-situ in $W \rightarrow e \nu$ events and in $Z \rightarrow \tau \tau$ events, where, using the mass constraint, the MET scale can be determined with an accuracy of $8 \%$.

The $Z+$ jets process, where the $Z$ decays to electrons and muons, is ideally suited to study the MET performance since it is almost background-free and the two leptons can be measured with excellent precision. This process can also directly be used for data-driven background estimations for processes involving MET. Semileptonic $t \bar{t}$ events have real MET and allow a test of the MET reconstruction in the high multiplicity environment relevant for SUSY searches. 


\subsection{Discovery at LHC}

The discovery of R-Parity-conserving SUSY is in principle "easy" at the LHC. In fact, squarks and gluino are produced through strong interactions, yielding cross-sections at the picobarn level for masses of order $1 \mathrm{TeV}$. Squarks and/or gluinos will then decay to the LSP through, in general, complex and model-dependent decay chains. The resulting final state will, in any case, include high $p_{T}$ hadronic jets, and missing transverse momentum from the two undetected LSPs in the final state. In most models, the decay chains will also involve the presence of leptons, $b$-jets, $\tau$-jets, photons, and $Z$ s.

There are, however, important experimental caveats. The essential point is that, although appropriate cuts will easily separate a low mass SUSY signal from the Standard Model backgrounds, the SUSY signal has no distinctive features, such as mass peaks, which separate it from the background. We are therefore dealing with counting experiments, where an accurate prediction of the backgrounds is mandatory.

An additional difficulty is the fact a large $E_{T}^{\text {miss }}$ can be generated by a number of experimental effects, and a complete control of this variable will require long and painstaking studies. Before a SUSY discovery can be claimed, a lot of work on understanding background will be needed, including both the control of experimental effects, and the collection of the needed control samples of Standard Model events. The time for discovery will be driven by these considerations rather than by the cumulated signal statistics. The ATLAS and CMS experiments have therefore recently focused their efforts on the development of methods based on a combination of Monte Carlo simulation and data-driven techniques for background estimation. The aim is to optimise, for each given value of integrated statistics, the level of systematic uncertainty on background estimate. Many different approaches to this issue are documented in [2,132]. As an example, for cumulated statistics of $1 \mathrm{fb}^{-1}$, the ATLAS collaborations quotes an uncertainty on backgrounds from mismeasured QCD jets of 50\%, and an uncertainty of $20 \%$ from backgrounds with real $E_{T}^{\text {miss }}$ from neutrinos, such as $\bar{t} t$ and $W+$ jets.

Based on these considerations, the ATLAS and CMS Collaborations have evaluated their SUSY discovery potential for inclusive analyses requiring high- $p_{T}$ jets, $E_{T}^{\text {miss }}$, and one or more additional leptons or other objects. The resulting reaches of the ATLAS and CMS experiments are given in terms of the parameters of a constrained SUGRA-inspired model, but the analyses are very general, and cover most of the topologies resulting from a generic MSSM with equivalent squark/gluino mass scale. With the moderate assumed luminosity of $1 \mathrm{fb}^{-1}$ squarks and gluinos with masses of order $1.3 \mathrm{TeV}$ should be discovered for a very broad range of models. For most of the accessible parameter space, SUSY should show up for several different signatures, thus enhancing the robustness of the signal. The quoted reach includes the systematic uncertainties on the backgrounds quoted above. It is expected that for lower luminosities the accuracy on background evaluation will decrease, severely affecting the discovery reach.

\subsection{Measurement at LHC}

Although hadron colliders have an excellent reputation for discovering new physics, the next step, that of measuring various properties associated with the new physics, is a very challenging one. The reasons for this are, of course, well known, and we shall not repeat them here. 
In the case of signals involving missing energy signals, the difficulties in performing measurements are compounded by the fact that kinematic information is lost in events: the energies and momenta of invisible particles go unmeasured. This problem can, to a certain extent, be offset by the fact that the total missing transverse momentum can be inferred from the measured transverse momentum in the event, but such a measurement in itself requires a very good global understanding of the detector.

\subsubsection{Mass measurements}

Assuming we discover a missing energy signal at the LHC associated with new physics, the first measurement priority will, presumably, be to establish the mass scale or scales of the new physics. In the absence of missing energy, such measurements are not difficult. Imagine, for example, that some new particle undergoes a decay into visible SM final states. To measure the mass of the new particle, it suffices to measure the four-momenta of the final states, to compute the invariant mass, and to look for a Breit-Wigner peak in the invariant mass distribution. The only major requirement is the ability to distinguish the signal amongst the background.

But for events with invisible particles, the final state cannot, in general, be reconstructed, and more thought is needed. In recent years, several methods to measure masses have been proposed. We discuss several of them below. In all cases, it turns out that it is far easier to measure mass differences between new states than to determine the overall mass scale of new physics. Unfortunately, it is the absolute mass scale that is of most interest to us, both in terms of our understanding of physics and in our planning for a future collider. It is, for example, the absolute mass scale that will be most relevant for dark matter, and for addressing the hierarchy problem (little or large). Similarly, it is the absolute mass scale that determines whether or not a future collider will be able to access the new physics.

\subsubsection{Invariant mass endpoints}

In cascade decays with at least two visible particles in the chain, one can construct various invariant mass combinations. For a particle of mass $m_{0}$ decaying to a massless visible state and an intermediate state of mass $m_{1}$, followed by a decay of the intermediate state into a second massless visible particle and a final invisible state of mass $m_{2}$, the endpoint of the visible invariant mass

distribution is given by $\sqrt{\left(m_{0}^{2}-m_{1}^{2}\right)\left(m_{1}^{2}-m_{2}^{2}\right) / m_{1}^{2}}$. So measurement of this endpoint yields one combination of the masses. In a cascade decay with $n$ visible particles, there are $n+1$ unknown masses and $2^{n}-n-1$ invariant mass distributions, so it is possible to determine all of the masses if $n \geq 3$. Assuming such cascades exist in Nature and can be isolated in experiment, the principal difficulties are to isolate the endpoints and also to solve the combinatoric issues of which of the two decay chains an observed particle belongs to, and where on the chain it belongs. For further discussion see [133-136].

\subsubsection{Polynomial constraints}

An alternative method [137-140] for cascade decay chains is to use the observed four-momenta directly rather than just the invariant masses. One can also combine the data from multiple events. If one isolates a single cascade decay with $n$ visible particles in each of $N$ events, then there are, as before, $n+1$ unknown masses and $4 N$ unknown momentum components (corresponding to the 
four-momentum of the LSP in each event). But energy conservation at each vertex implies that there are $N(n+1)$ constraints. The constrained system of polynomial equations can therefore be solved (up to discrete ambiguities) provided $n \geq 4$.

If on the other hand, one is able to observe both cascade decay chains in $N$ events (the chains are assumed to contain $n$ and $m$ visible particles respectively) then the number of unknown four-momentum components is only $6 \mathrm{~N}$, because even though there are two LSPs, two of the four-momentum components can be inferred from the missing transverse momentum. Counting up the constraints as before, one finds that the system of polynomial equations can be solved for $n+m \geq 5$; for identical chains with $n=m$, one has $n \geq 3$, as for the endpoint method. Even if one is in a situation with shorter cascade decays and an underconstrained system, one can still use the constraints from many events to pin down the masses [141].

The advantage of methods of this type is that, unlike the endpoint methods which employ only a subset of events (those near the endpoint) to perform a measurement, the polynomial methods use all of the available data. The disadvantages are that the solutions one obtains by solving the system are often the wrong ones, or simply do not exist, because of combinatoric ambiguities and mismeasurement effects. Moreover, it is not clear that these methods, which combine pool together a small number of events at a time until an exactly constrained system is obtained, are the best way to combine data from many events.

Is there a better way? According to the theory of statistics, this situation of partial data from multiple events is naturally dealt with using the likelihood function. The problem in the case at hand is that the likelihood should be constructed from the matrix element, and since the new physics is unknown, the matrix element is also unknown. Short of prescribing the new physics, the best hope seems to be to define the likelihood in an ad hoc way [142,143].

\subsubsection{Transverse observables}

These methods are all based on method originally used to measure the mass of the $W$-boson in its leptonic decays after its discovery at UA1 and UA2. This method is still used today at the tevatron [144], and provides the single most precise measurement of the $W$ mass. In the leptonic decay of a $W$, the neutrino is invisible in the detector. The transverse mass, defined by

$$
m_{T}^{2} \equiv m_{v}^{2}+m_{i}^{2}+2\left(e_{v} e_{i}-\mathbf{p}_{v} \cdot \mathbf{p}_{i}\right),
$$

where $\mathbf{p}$ is the momentum transverse to the beam, $e=\sqrt{\mathbf{p} \cdot \mathbf{p}+m^{2}}$ denotes the transverse energy, and $v$ and $i$ label the visible and invisible decay products respectively, (a charged lepton and a neutrino in the case at hand), is an observable, because $m_{i}$ can be neglected and because $\mathbf{p}_{i}$ can be inferred from the missing transverse momentum in the event. Moreover, $m_{T}$ is bounded above by $m_{W}$, and so its distribution features at edge. In practice, the edge is smeared out by resolution and finite-width effects, but provided that this can be modelled, a good measurement of the $m_{W}$ can be obtained.

In the case of missing energy events associated with new physics, two complications arise. The first is that, since the invisible particles are typically pair produced, the individual transverse momenta of the invisible particles cannot be inferred. This problem has been solved by the introduction of the derived observable $m_{T 2}[145,146]$. (Very recently, the usefulness of $m_{T 2}$ for hadron collider measurements has been confirmed in its application to measurement of the top quark mass 
in the dileptonic decay channel at the Tevatron [147].) The second problem is that the masses of the new, invisible particles cannot be neglected, so $m_{T}$ is no longer observable. At way out of this impasse has been found, at least in principle, in [148-151]: one can compute the distribution of $m_{T}$ or $m_{T 2}$ for some hypothetical value of the invisible mass; a plot of the endpoints of the resulting distributions, considered as a function of the hypothetical mass, features a kink (is continuous, but not differentiable) exactly at the point where the hypothetical mass equals the true invisible mass. The co-ordinates of the kink this yield the masses of both the parent and the invisible daughter. If one generalizes to more complex decay topologies, one finds that all of the unknown masses can be determined $[152,153]$

It was pointed out in [154], that this method is connected to the polynomial approach in the following way: For each event, the $m_{T 2}$ variable, considered as a function of the unknown LSP mass, generates a curve which delineates the boundary of the region in mass space which is compatible with the kinematic constraints. This shows that the methods described in [148-151] are in fact the best one can hope for using kinematic information alone.

Whether or not this method can actually be successfully employed in the real-world remains to be seen. What is clear is that this is the only known method that works for any decay topology. In particular, it is the only method that exists to measure the masses in two-body decays, or decays involving off-shell intermediate states.

In another development, it has been shown in $[155,156]$ that observables of this type may be of some use for discovery itself, rather than for the later task of mass measurement. Finally, we note that various other methods involving transverse observables of one kind or another have also appeared [157-160].

\subsubsection{Spin measurements}

Spin measurements will be crucial in determining the nature of the new physics. In particular, various scenarios for physics beyond the SM involve new particles which are partners of the SM particles, in that they share the same quantum numbers as SM particles. They differ in their spins, however. For example, in supersymmetric scenarios, the SM partners, or rather superpartners, have spins which differ by one-half from the corresponding SM states. In extra-dimensional scenarios, by contrast, the spins are the same.

Just as for mass measurements, spin measurements are complicated by the presence of missing energy in the final state. Roughly speaking, the reason for this is that spin, being a form of angular momentum, is a generator of rotations. Thus the physics of spins is principally associated with the angular distribution properties of production and decay processes. But in order to measure these angles, one needs to be able to reconstruct some reference frame, such as the rest frame of a decaying particle, or the centre of mass frame in a two-body collision. But the presence of missing energy makes reconstruction very difficult. For a recent review of spin measurement at the LHC, see [161].

\subsubsection{Cascade decays}

Spin measurements can still be performed, even if one is not able to reconstruct reference frames. For example, if one looks at Lorentz-invariant quantities, the choice of frame is irrelevant. This observation gives rise to the first method for spin measurement [162-168], which looks at the in- 
variant mass distributions of various visible final state particles. The invariant masses do depend on the angular properties of decays, and do therefore contain information about the spin, in principle. In order that non-trivial angular correlations exist, one requires at least that the intermediate particle in question be polarized, and, if a fermion, that its decay be chiral [169].

\subsubsection{Production processes}

One can also hope to perform spin measurement via angular effects in production processes. The main difficulty here is that, at a hadron collider, many sub-processes, each with different angular dependence, contribute in a given production channel. A method for measuring the slepton spin in dislepton production via $q \bar{q}$ production via a $Z / \gamma$ was proposed in [170]. One exploits the fact that the pseudo-rapidity of the resulting leptons is correlated with the $Z / \gamma$ decay angle. The viability of this method depends strongly on the mass spectrum. Measurement of the slepton spin should be much easier in a future lepton collider [171-173].

A second option is to look at the azimuthal angular effects in production $[174,175]$. These effects arise whenever multiple sub-processes are able to interfere.

A third method allows one to measure the spin of pair-produced gluinos decaying to $b \tilde{b}$ [176], by looking for asymmetries in the tagged $b$ and lepton system. One may also look at the shape of the dijet mass distribution in $q \tilde{q}$ decays [177].

\subsubsection{Three-body decays}

In a situation where new particles undergo point-like three-body decays (involving one invisible particle in the final state), two methods have been proposed. One is to simply look at the invariant mass distribution of the two visible final state particles [178]. The other [179] uses the $m_{T 2}$ variable, discussed above in relation to mass measurement, to assign the four-momentum of the invisible particle. The distribution of assigned momenta is peaked around the true value. Once the momentum has been assigned, one can construct both invariant mass distributions and the Dalitz plot, from which the spin can be inferred. It is worth remarking that this so-called 'MAOS' (for $m_{T 2}$ assisted on-shell) method, which enables one to reconstruct in a statistical fashion the final state and, ergo a frame of reference, should be of much wider applicability.

\subsubsection{Cross sections}

The last method [180-182] we mention simply utilizes the fact that the production cross sections for particles have a strong dependence on the spins of the particles involved. Unfortunately, cross sections also depend strongly on masses, so precise mass measurements will be necessary prerequisites to such a method. This is exacerbated by our inability to measure or model cross sections precisely at hadron colliders.

\subsubsection{Polarization measurements}

One idea, in a supersymmetric scenario, is to measure the polarization of the tops arising from stop pair production followed by decays to the top and the lightest neutralino [183]. The top polarization may be inferred from the distribution of the various decays products of the top, be they hadronic or leptonic. If measurable, the polarization would yield information on the mixing angle between the 
right- and left-handed stops. This angle is of some interest in connection with the little hierarchy problem [184].

For methods based on the $\tau$ polarization, see, e.g. [185-188].

\subsubsection{Measurement of ratios of branching ratios}

The other promising observable for the determination of the stop mixing angle $\cos \theta_{\tilde{t}}$ turns out to be the ratio of different branching ratios in stop decays. In [124] it has been shown that the different branching ratios of stop decays are sensitive to the mixing angle. In [189] it is discussed that $\tilde{t}_{1}$-decay into $\tilde{\chi}_{1}^{ \pm}$and $\tilde{\chi}_{1,2}^{0}$ can be a sensitive probe of the stop mixing angle and in some cases also of the CP-violating phase. The origin for such sensitivity is the different coupling structure of $\tilde{t}_{R}$ and $\tilde{t}_{L}$ to gauginos and higgsinos. Since the measurement of the branching ratios can turn out to be very challenging, the ratio of different branching ratios is more promising. Defining

$$
R_{1}=\frac{B R\left(\tilde{t}_{1} \rightarrow \tilde{\chi}_{1}^{+} b\right)}{B R\left(\tilde{t}_{1} \rightarrow \tilde{\chi}_{1}^{0} t\right)}, \quad R_{2}=\frac{B R\left(\tilde{t}_{1} \rightarrow \tilde{\chi}_{1}^{+} b\right)}{B R\left(\tilde{t}_{1} \rightarrow \tilde{\chi}_{2}^{0} t\right)}, \quad R_{3}=\frac{B R\left(\tilde{t}_{1} \rightarrow \tilde{\chi}_{1}^{0} t\right)}{B R\left(\tilde{t}_{1} \rightarrow \tilde{\chi}_{2}^{0} t\right)}
$$

as observables and using 1000 events of stop production, a $\chi^{2}$-fit has been performed for the scenario SPS1a' to all 3 ratios. One obtains a two-fold ambiguity that needs imput from other measurements and observables to be resolved. Assuming the correct solution can be pinned down, this leads to a determination of the mixing angle and of the stop mass

$$
\begin{aligned}
& \cos \theta_{\tilde{t}}=0.56 \pm 0.03, \\
& m_{\tilde{t}_{1}}=366 \pm 3 \mathrm{GeV},
\end{aligned}
$$

where 1- $\sigma$ statistical error has been included so far [189], see Fig. 3.5. Further studies are needed that will take into account pollution from background and experimental effects.

\subsubsection{On-Shell Effective Theories}

All the methods discussed above rely on kinematic properties, and are thus largely model-independent. This is perhaps just as well, given that we don't yet know what the new physics is. Nevertheless, kinematics can only take us so far; at some point we will want to introduce a model and compare it to the data. Indeed, the nature of a hadron collider, with large backgrounds and slowly-varying distributions, makes it rather likely that we will have to do this sooner rather than later in our analysis of the data.

The question then is: what model should we choose? The traditional paradigm in particle physics has been to specify a lagrangian, which of course provides a complete description of the physics. Unfortunately, the lagrangians that have been suggested for physics beyond the SM are typically very complex, involving many new fields and often hundreds of free parameters. Even though such a Lagrangian may well describe the LHC data, one may wonder whether such a complicated description is something of an overkill, given the fact that measurements at the LHC will neither be particularly precise, nor wide-ranging.

One recent suggestion has been to use so-called 'On-shell effective theories' [190] as a more simplistic description of new physics than a Lagrangian. To define a model of this type, one 

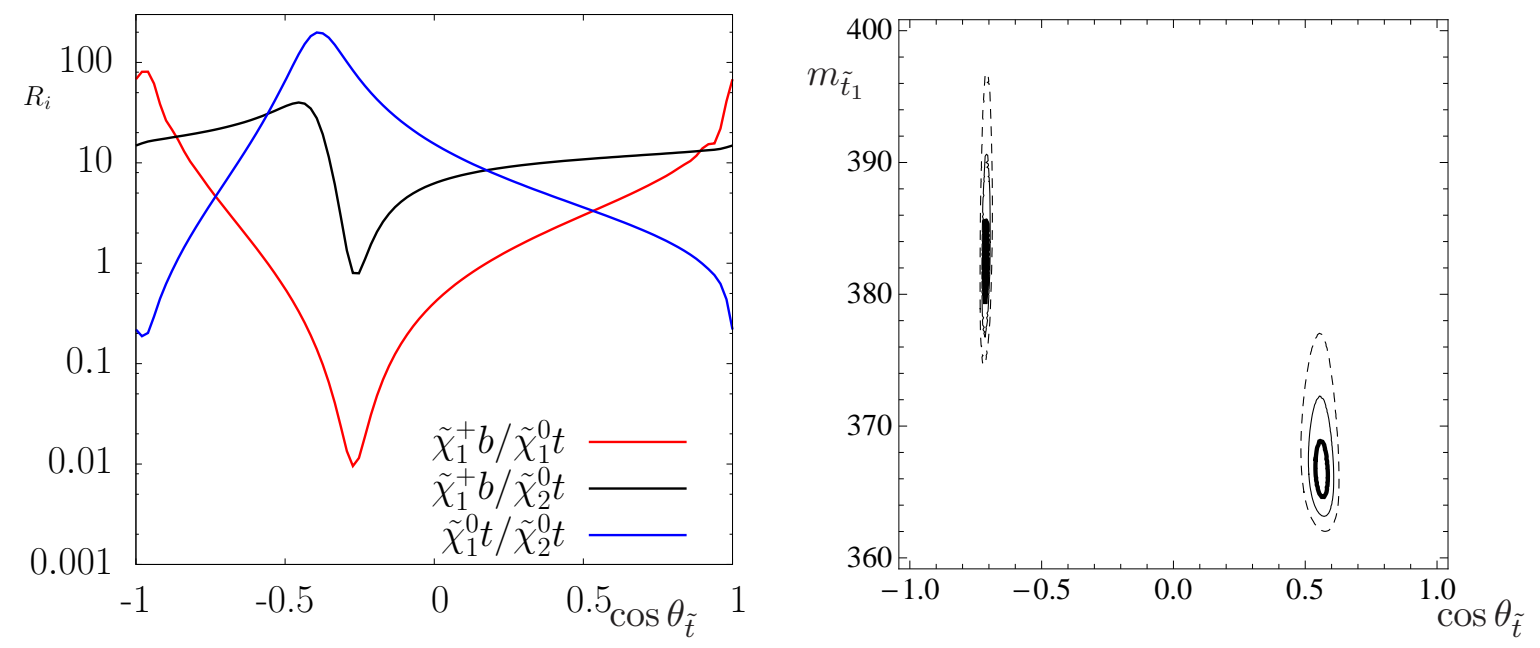

Fig. 3.5: Ratios of different branching ratios (left panel), Eq. 3.2, in scenario SPS1a' as a function of the stop mixing angle $\cos \theta_{\tilde{t}}$. The $\chi^{2}$-test (right panel) leads to a determination of the stop mixing angle at the $5 \%$-level and of the mass at the 1\%-2\%-level [189] (assuming 1000 well identified events). The bold, solid and dashed lines denote the $1 \sigma, 2 \sigma$ and $3 \sigma$ contours, respectively.

needs only to specify the particles, as well as the principal channels by which those particles are produced and decay. The particle masses and the production cross sections and decay branching ratios are included as free parameters to be fitted to the data. The initial results seem reasonably encouraging, with indications that masses can be determined to $O(50 \mathrm{GeV})$ or so. Clearly, as the number of observed production and decay channels proliferates, the utility of such a method diminishes rapidly, but at least in the early period of LHC data, this approach may well turn out to be of use, at least as a stepping-stone in guiding us towards the right Lagrangian.

\subsubsection{Numerical Tools}

A number of numerical analysis tools were presented during the workshop including: Sfitter [191] and Fittino [192] for MSSM fits; Gfitter [48] for global electroweak fits; MasterCode [53,193, 194] for fits using the combination of all current experimental data; fits to the constrained MSSM using Bayesian [195-197] or frequentist [198-201] approaches, see also sect.3.6.1.1.

\subsection{The LHC-Dark Matter Connection}

The Dark Matter (DM) relic density has been measured with very high precision by the WMAP experiment [202]. In order to interpret the measurement in terms of particle physics, a large effort is being devoted to experiments for the direct detection of a DM particle candidate. An alternative approach would be to produce the DM particles with high energy accelerators. Given a DM candidate, its relic density can be calculated from its mass and from the cross section for its annihilation [203,204]. Several public programs [205-207] are available to perform these calculations, and have been presented during the workshop. A basic result is that, in order to account for the 
observed relic density, a DM candidate with a mass scale of $100 \mathrm{GeV}$ and weak annihilation crosssection is needed. This is a remarkable result, which points to the possibility of identifying the DM candidate with the stable weak-interacting particle that terminates the cascade decays of several new physics models. A typical example is the LSP in the MSSM. If all the parameters defining the BSM model can be measured at a combination of future high energy colliders, the annihilation rate of the DM candidate particle can be calculated, and the relic density predicted, based on the collider data.

Concentrating on SUSY MSSM, a natural question is whether the constraints on the MSSM parameter space achieved thorough the measurements discussed in the previous sections can significantly constrain the relic density. This can be done either based on the constraints on the model parameters calculated based on the numerical tools described in section 3.4.6, or directly based on the measurements we expect to be able to perform at colliders for a given benchmark point. We find in the literature two exercises which explore the latter approach in great detail, based on a version of the MSSM with around fifteen parameters. The work of [208] is focused on a very favourable SUSY point and restricted to LHC data, whereas the work in [209] is of broader scope, addressing several different final-state topologies and exploring both the potential of the LHC, and the combination of the LHC and a future Linear Collider. The result is that the LHC data alone can give a well defined prediction only for one of the studied topologies, the case corresponding to neutralino annihilation through exchange of a light selectron or smuon, whereas in all other considered cases a combination of LHC and linear Collider measurements will be needed. Another recent study [210] shows, for instance, how accurate the gaugino component of the dark matter candidate has to be determined in order to avoid substantial misinterpretations, cf. Section 3.6.1.1. Such cases of parameter points impose a big challenge for LHC predictions and further input from a linear collider will be required. This is a line of investigation which deserves further investigation, as its success would effectively unify the fields of particle physics and cosmology.

\subsection{Future Lepton Colliders}

\subsubsection{Introduction and characteristic features at linear colliders}

Due to the clean environment, unbiased measurements with high precision are expected to be only achievable at a future linear collider. Therefore linear collider physics will perfectly complement and extend the physics potential of the LHC [124] and a strong physics case can already be derived from present knowledge. The discovery potential of a linear collider is not only defined by its energy scale but in particular by the precision of its measurements.

As one example for the need of precision measurement that is well-defined already today is

physics of the top quark. Applying threshold scans for top quark mass measurement a precision of $m_{t}=100 \mathrm{MeV}$ is predicted, see [211]. The measurement of the top mass is of utmost importance for the electroweak precision observables and predictions of the Higgs sector, since $m_{t}$ enters up to $m_{t}^{4}$ at the quantum level. It causes intrinsic theoretical uncertainties that will only be matched with a measured $\Delta m_{t}$ at the linear collider [212]. Concerning the strong linear collider physics potential in the top and Higgs sector, for instance for measuring absolute couplings, branching ratios and widths, see $[211,213]$. Due to the broad spectrum of different experimental analyses and the unprecedented potential for precision measurements at the linear collider, it is hard to 
imagine any LHC result that would not require an extensive $e^{+} e^{-}$physics programme [211] (see for instance [40] and references therein).

Characteristics of a linear collider are the precisely defined and known centre-of-mass energy, the clean environment, the possibility to avoid any hardware trigger, and the ability to fully reconstruct both leptonic and hadronic final states. The additional features of providing a precise tunable energy together with polarized beams open a broad spectrum of available different analyses and prepare the LC ideally for the 'unexpected'. Threshold scans allow most precise mass measurements and open up also experimental possibilties for probing the existence of CP-violation, for instance, in the chargino sector of SUSY. Another spectacular experimental tool is the availability of the polarization of both beams which has a rich field of physics applications [214]. It offers, for instance, unique access to the chiral structure of all kind of interactions and strengthens strongly the potential to perform model-independent analyses.

\subsubsection{Parameter determination in the SUSY sector}

Any model-independent determination of parameters in new physics models as, for instance, in SUSY, is expected to be achievable only in the clean linear collider enviroment. Applying the full programme of polarized beams in their different configurations, threshold scans and continuum measurements and in combination with LHC results on the coloured spectrum it may even be possible to extrapolate the high-energy behaviour of new physics and, for instance, perform tests of the SUSY breaking mechanisms in supersymmetric and string models [215]. The programs Fittino [192] and Sfitter [191], see sect. 3.4.6, determine SUSY parameters in a multi-parameter fit for several models, including extended SUSY models. The strong impact of linear collider results when combined with results from the LHC at an integrated luminosity of $300 \mathrm{fb}^{-1}$ for the determination of the parameters in the MSSM with 18 parameters can impressively be seen in Fig. 3.6, [216].
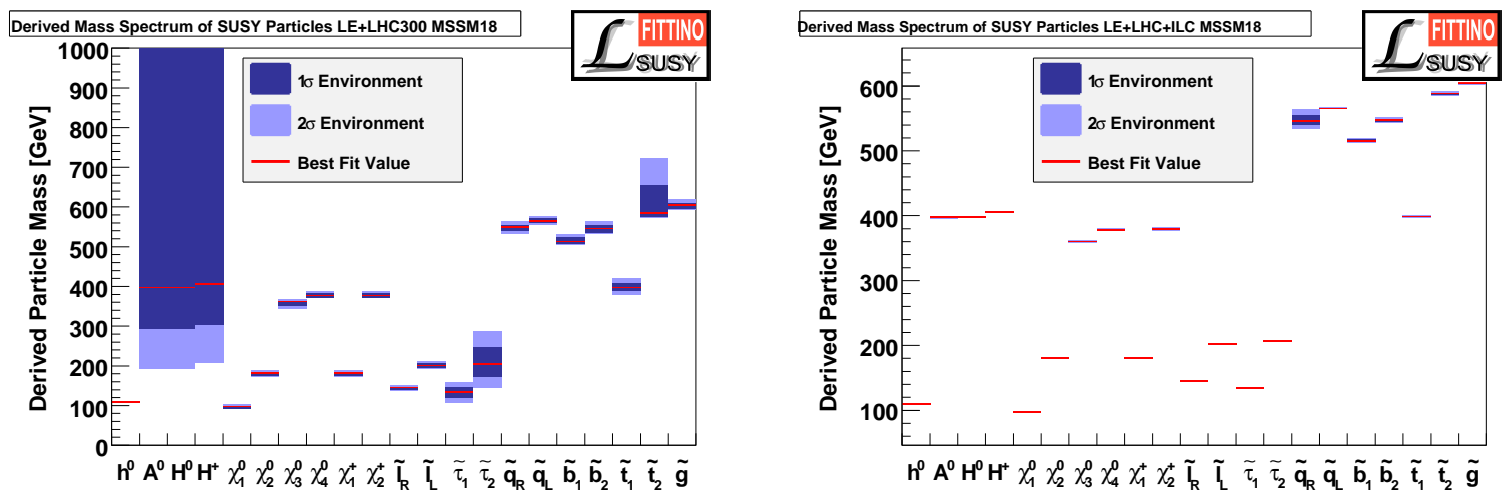

Fig. 3.6: Left panel: SUSY mass spectrum derived from low-energy observables and expected LHC measurements at $\mathcal{L}^{\text {int }}=300 \mathrm{fb}^{-1}$ for the MSSM18 model. The uncertainty ranges represent model dependent uncertainties of the sparticle masses and not direct mass measurements [216]. Right panel: Derived mass distributions of the SUSY particles in the MSSM18 model, using low energy observables, expected results from LHC with $\mathcal{L}^{\text {int }}=300 \mathrm{fb}^{-1}$ and expected results from ILC [216]. 

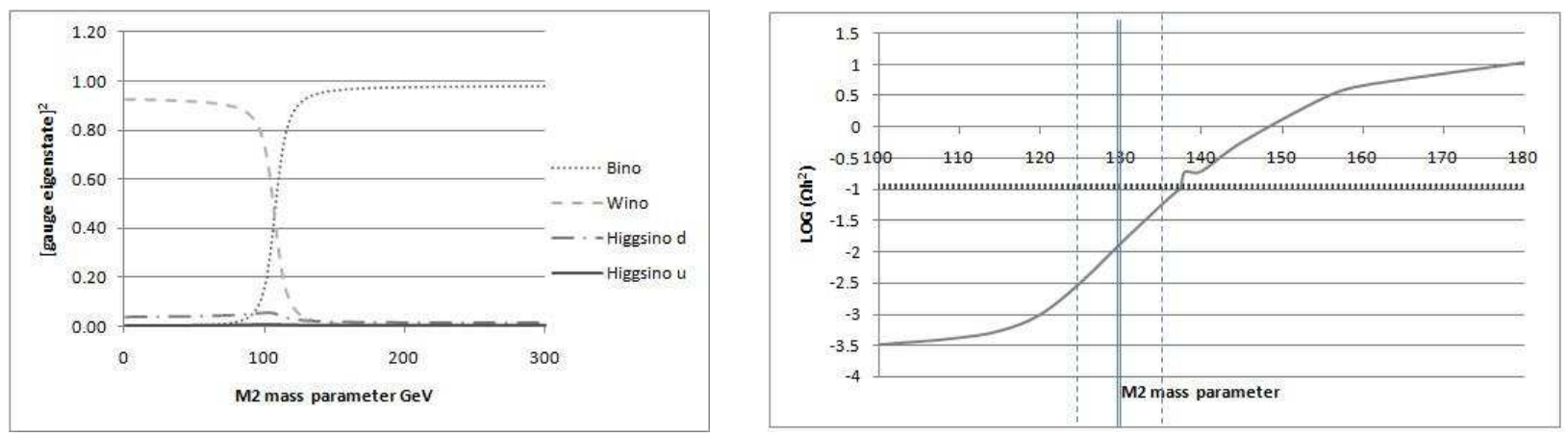

Fig. 3.7: Left panel: The gaugino and higgsino components of the LSP in SPS1a' scenario where only the $M_{2}$ parameter is varied. Around $M_{2} \sim 104 \mathrm{GeV}$ the mixing character of the LSP changes abruptly due to the swapping of the mass eigenstates. Right panel: the corresponding change in the dark matter contribution of the LSP for this scenario with variation of $M_{2}$ [210].

\section{Dark matter predictions at required accuracy}

Accurate parameter determination is also crucial for testing the dark matter properties of the new particles. With entering the cosmology in an era of precision measurements, it might be possible to match results from missing energy signals with precise dark matter predictions. However, high precision in the model-independent determination of the dark matter gaugino eigenstate is necessary to avoid severe misinterpretations in parts of the SUSY parameter space where, for instance, the LSP changes abruptly its interaction character [210]. Such abrupt changes can happen due to the swapping of the mass eigenstates. As shown in Fig. 3.7 (left panel) for a general MSSM model without unfication assumptions, a high precision on the measurement of $M_{2}$, only achievable at the linear collider, is required. Such a precision is crucial in order to reliably test whether the measured SUSY scenario leads to a contribution of $\tilde{\chi}_{1}^{0}$ that is consistent with the WMAP bounds or not, see Fig.3.7(right panel) [210]. For some further highlights of expected physics results at the linear collider, see $[40,211]$ and references therein.

\subsubsection{Technical design status and technology options}

Two accelerator technologies are discussed for a linear collider.

a) In the energy range of $\sqrt{s}=0.5-1 \mathrm{TeV}$ the superconducting technology, as implemented in the International Linear Collider (ILC), is the mature concept [217] to provide the expected unique scientific opportunity and enter a new precision frontier. The Reference Design Report (RDR) has been finished in 2007 [218]. No technical obstacles are predicted for the ILC design, but careful studies of possible cost saving changes for the current design have to be done with regard to their possible impact on the physics potential of the machine. The Technical Design Phase (TDP) of the ILC is under the responsibility of the 'Global Design Effort' (GDE).

Starting the industrial engineering phase, the optimization of, for instance, the cavities shapes are under study. Higher gradients up to $59 \mathrm{MV} / \mathrm{m}$ have already been achieved in single cells with so-called 're-entrant' cavities, developed by Cornell and KEK. Concern- 
ing the industrial cavity production, an average of $36 \mathrm{MV} / \mathrm{m}$ in nine-cell cavities has been achieved [219].

b) Further exploitation of new physics scenarios as well as the more precise determination of the electroweak symmetry breaking mechanism may require a linear collider design with a higher cms energy in the multi-TeV range as foreseen for the CLIC design [220] (see also Sect. 1).

High mass particles in the range beyond $1 \mathrm{TeV}$, e.g. heavy squarks in SUSY, can be studied at CLIC, should they be discovered at the LHC or inferred at the ILC. It is also expected to determine the triple Higgs couplings that are important for the verification of the Higgs mechanism about a factor 2 more precisely at higher cms energy than at $500 \mathrm{GeV}$ if a similar clean experimental environment is achievable at the multi-TeV option.

For achieving higher energies of $\sqrt{s}$ in the multi-TeV range a normalconducting two-beam acceleration concept is discussed, the Compact Linear Collider (CLIC). A conceptual design report is foreseen for 2010, where the key feasibility issues of the CLIC technology are foreseen to be demonstrated as well as the preliminary performance and a first cost estimation.

A fruitful ILC/CLIC collaboration has been started to address common R\&D issues in the civil engineering \& conventional facilities, beam delivery system, beam dynamics and detectors, more details see [221]. Comparing the potential of the two linear collider technologies several technical issues have to be taken into account that may have impact on the physics potential. Many precision measurements at a linear collider depend crucially on machine parameter more than on the achievable detector precision. For instance, the average energy loss i.e. beamstrahlung, has impact on the precision achievable via threshold scans and (polarized) cross sections. Beamstrahlung is predicted to be $2.4 \%$ at ILC with $\sqrt{s}=500 \mathrm{GeV}$ (ILC500), 7\% at CLIC with $\sqrt{s}=500 \mathrm{GeV}$ (CLIC500) and 29\% at CLIC technology with $\sqrt{s}=3000$ (CLIC3000). A formidable experimental challenge arises from the short $(0.5 \mathrm{~ns})$ bunch spacing at CLIC. Severe impact on the achievable precision due to pile-up of soft hadronic interactions can arise unless unprecedented time-stamping capability both for charged and neutral particles can be implemented into the CLIC detectors.Detailed simulations will be needed for achieving conclusive results concerning the physics potential of the different designs. Therefore a staged approach between the different design may be beneficial [211].

\subsubsection{Impact of early LHC results on the LC}

Important information from early LHC results could infer technical but also theoretical requirements for linear collider physics. Sensitive science areas in this context are, for instance:

1. defining further specific LC detector capabilities,

2. providing a way out of worst case scenarios in the interpretation of LHC data

3. staging the required energy steps at the LC, optimizing the running scenarios and outlining linear and hadron collider upgrade options.

\subsubsection{Specific detector capabilities}

Results from LHC data could have an impact on the required ILC detector capabilities. For instance, if one finds hints for the existence of new CP-violating phenomena as expected in supersymmetry, high resolution in the capabilities of $b$ - and $c$-charge tagging may be required for the LC detectors in order to optimize respectively the physics potential of the ILC. 

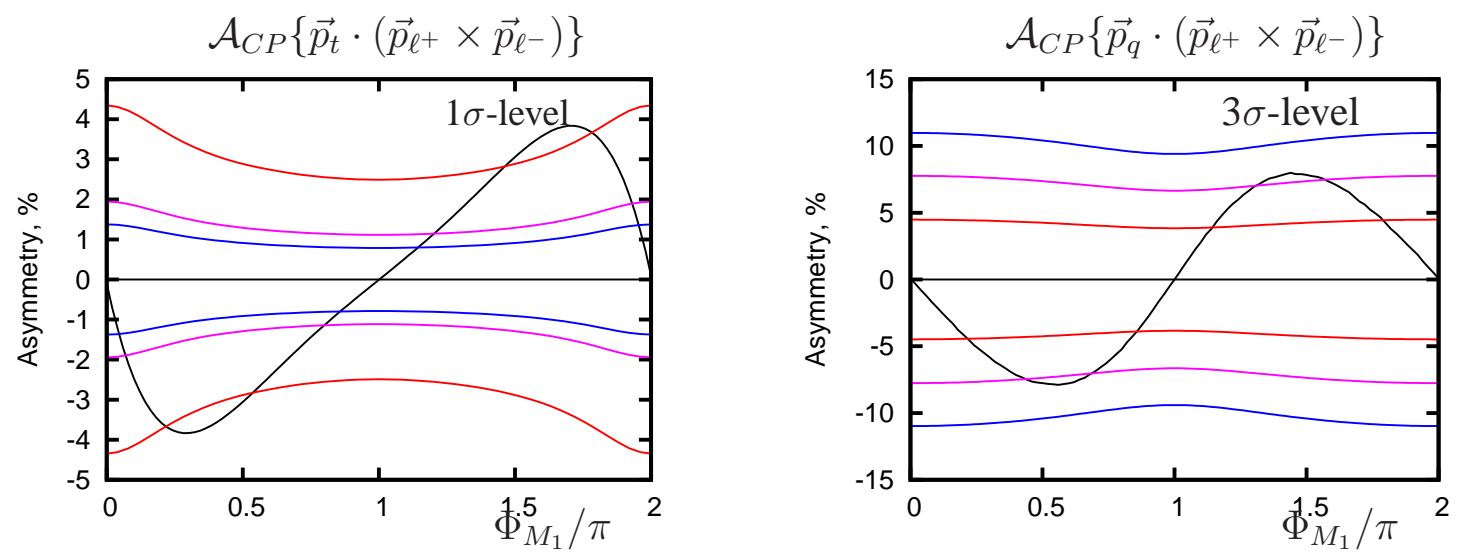

Fig. 3.8: Left panel: CP-odd asymmetry sensitive to $\Phi_{M_{1}}$ in stop decays at the LHC with parton density functions included in the production process at the $1 \sigma$-level with the luminosities, $\mathcal{L}=\left(100 \mathrm{fb}^{-1}, 500 \mathrm{fb}^{-1}, 1\right.$ $\mathrm{ab}^{-1}$ ) [227]. Right panel: CP-odd asymmetry in squark decays with parton density functions included in the production process but after momentum reconstruction has been performed. The selection cuts have been applied and the momenta of the final state particles have been smeared to replicate the LHC detectors effects. The coloured lines show the size of the asymmetry needed for a $3 \sigma$ observation at the given luminosity, $\mathcal{L}=\left(50 \mathrm{fb}^{-1}, 100 \mathrm{fb}^{-1}, 300 \mathrm{fb}^{-1}\right)[229]$.

Since some of the CP-violating phases are strongly experimentally constrained by the electric dipole moments of the electron, neutron and some atoms, only small phases are expected in the SUSY sector, although large phases can still be accomodated in the free parameter space of the MSSM, see [222-225] and references therein. Hints for unique signs of CP-violation may be difficult to detect in the harsh environment of the LHC. It has been studied in [226-229] how to detect the effects of SUSY CP-phases in squark decays into neutralinos at the LHC.

In [227] CP-odd sensitive observables, i.e. asymmetries composed by triple product correlations, have been analyzed in detail for the process $g g \rightarrow \tilde{t}_{1} \tilde{t}_{1}, \tilde{t}_{1} \rightarrow t \tilde{\chi}_{2}^{0}, \tilde{\chi}_{2}^{0} \rightarrow \tilde{\chi}_{1}^{0} \ell^{+} \ell^{-}$and $t \rightarrow W b$ at parton and at hadronic level. Although this process offers several asymmetries that show different sensitivity to CP-phases, so that effects of $\Phi_{M_{1}}$ and $\Phi_{A_{t}}$ might be distinguishable, the statistics are quite low. Even in the case of large asymmetries up to $\mathrm{O}(10 \%)$ at the parton level, they are significantly reduced by about a factor 4 due to dilution at the hadronic level. Therefore high luminosity is desired, see Fig. 3.8. Studying CP-effects in the process, $q g \rightarrow \tilde{q}_{L} \tilde{g}, \tilde{q}_{L} \rightarrow q \tilde{\chi}_{2}^{0}$, $\tilde{\chi}_{2}^{0} \rightarrow \tilde{\chi}_{1}^{0} \ell^{+} \ell^{-}$leads to a significantly higher statistics and although the dilution reduces the asymmetry again by about a factor 3-4 when going from the partonic to the hadronic level, a large range of $\Phi_{M_{1}}$ may lead to observable asymmetries even at the $3 \sigma$-level, in particular after momentum reconstruction of the LSP has been done, see Fig. 3.8 (right panel). Some selection cuts and smearing effects at the LHC have already been included [229].

Another example where LHC results may have an impact on the optimization of the LC detector are physics scenarios that require to measure precisely the polarization of $\tau$ 's [230,231] in order to, for instance, decompose the caracter of the lightest SUSY particle and provide model distinction of different physics scenarios [232], aim to determine the SUSY $A_{\tau}$ parameter [233] or to test $C P$ quantum numbers in the Higgs sector [234,235]. 
Particularly challenging scenarios of new physics consist of new particles that are almost mass-degenerate. In case that LHC results point to SUSY scenarios where a close mass degeneracies of the light neutral and charged gauginos, $\tilde{\chi}_{1}^{ \pm}, \tilde{\chi}_{2}^{0}$ is expected, one needs an excellent jet energy resolution in the LC detector in order to resolve whether the final di-jet pair originates from a $W^{ \pm}$or a $Z^{0}$ decay [236] and the LC detector might be optimized correspondingly.

\subsubsection{Worst case scenarios from early $L H C$ results}

Although hints from electroweak precision observables point to a rather light scale of new physics as, for instance, in supersymmetry [194,216], it may happen that nothing new or only a light SMlike Higgs has been detected within early LHC data. In such a worst case scenarios what may be the conclusions for collider physics?

If really no hints for new physics can be seen at early LHC data it is mandatory to exploit as soon as possible the top quark with highest priority. This particle plays a key role in the understanding of the electroweak breaking mechanism since it enters via loops effects at the quantum level. The first energy stage at a linear collider may therefore not be $\sqrt{s}=500 \mathrm{GeV}$ but the $m_{t}$ threshold of about $\sqrt{s}=350 \mathrm{GeV}$. Such a reduction for the required LC energy in its first stage has, of course, a strong impact on cost issues of the LC. Another high priority physics in such a worst case scenario will be the exploitation of electroweak physics at the Z-pole. Only high luminosity at the Z-pole is required to achieve an unprecedented precision in the measurements of $\sin ^{2} \theta_{\text {eff }}$. Even only small traces of new physics contributions in the electroweak sector can be detected via this observable. One should remember that although a deviation of the measured $\sin ^{2} \theta_{\text {eff }}$ from its SM prediction does not specify the new physics model, but it would, however, give evidence for physics beyond the Standard Model. Such an information may be crucial for continuing collider data analysis at the highest precision level.

In case the early LHC data detect only a SM-like Higgs signal but nothing else (see also Sect. 1 for a discussion), the foreseen first energy stage of a LC has to be questioned. Running at the threshold for $Z^{0}+$ Higgs production would probably offer cost savings as well as provide a tremendous benefit for physics. In such a scenario, it is also advantageous to perform a high luminosity run at the Z-pole instead of aiming at higher energies in the first stage of the LC. For such worst case scenarios, studies of $\sin ^{2} \theta_{\text {eff }}$ at the quantum level have been made. These studies clearly demonstrate that a high precision measurements of $\sin ^{2} \theta_{\text {eff }}$ could point to the existence of supersymmetry, even if the coloured SUSY particles were in the multi-TeV range and not detectable at the early LHC [214,237], see Fig. 3.9. Furthermore, such high precision measurements would indicate the new physics scale and point to the required energy stage of the LC.

\subsubsection{Impact on running scenarios and upgrade options}

In case new physics is detected in early LHC data, this important information can be used to optimize the different LC running scenarios and future collider options. Given that a new physics scenario with many new particles has been detected, the technical potential of a linear collider to perform threshold scans can be optimized. The most precise mass measurements as well as hints for CP-violation as, for instance, in SUSY are available by measuring the corresponding threshold behaviour. However, each threshold scan uses a specific amount of the total integrated luminosity and therefore not an arbitrary number of scans can be done. Therefore optimization of threshold scans via defining the suitable energy steps for the scans as well as selecting the most crucial scans 


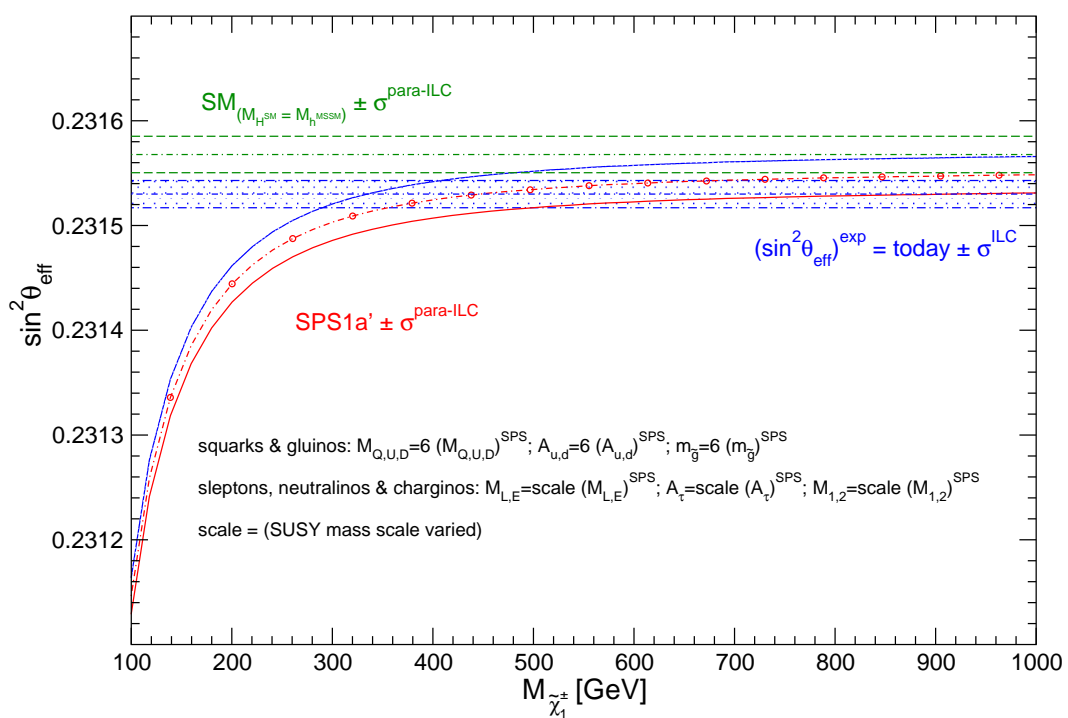

Fig. 3.9: Theoretical prediction for $\sin ^{2} \theta_{\text {eff }}$ in $\operatorname{SM}$ and MSSM compared to future ILC precision [214,237]. The SUSY scales are varied with a common scalefactor, but squark and gluino masses are fixed to be heavy, i.e. not observable at the LHC. The anticipated parametric uncertainty of ILC is indicated as part of the theory predictions. The plot shows the sensitivity to contributions of $m_{\tilde{\chi}_{1}^{ \pm}}>\sqrt{s} / 2$ in such worst case scenarios.

to derive the maximal information on the new physics sector are the key issues of the LHC input in such cases.

Another important LC sector where immediate input from LHC data is important is narrowing the choices for the different upgrade options of a linear collider. In some cases, the most efficient way may be to go straight to the $\gamma \gamma$ option but -as pointed out in the paragraphs abovethere may also be cases where the GigaZ option is the best solution. Synergy between early LHC data and a LC with a first energy stage of $\sqrt{s}=500 \mathrm{GeV}$ may also be crucial to predict the needed energy scale for energy upgrades of the LC. For instance, even in SUSY scenarios where only light gauginos/higgsinos may be accessible but the sfermion sector is within the multi-TeV range, the combined interpretation of early LHC results together with the precision measurements at the $500 \mathrm{GeV}$ LC allow a model-independent determination of the fundamental MSSM parameters and enable a rather accurate prediction of the masses of squarks and sleptons in the multi-TeV range [238]. The required scale for a possible multi-TeV LC option can therefore successfully be envisaged, based on early LHC and LC results. Another example where LHC data may influence the LC running scenarios concerns the suppression of background processes of new physics. For instance in SUSY, the most severe background processes are expected to be supersymmetric background processes themselves. Therefore a crucial information from LHC data could provide substantial information on choosing the optimal configuration of $e^{+} e^{-}$beam polarization in order to reduce the background most sufficiently.

There exist also scenarios in supersymmetry where only the $e \gamma$ and $e^{-} e^{-}$options of a LC would lead to a significant improvement in SUSY analyses. For instance, in cases with heavy selectrons, where at least a single production may kinematically be accessible within these collider options. Weighting these options with regard to a total life time of a future LC, however, it is 
mandatory to fold in all possible information coming from early LHC data in order to maximally exploit the full potential of a LC with all its variable running options. Many of these collider options also have impact on costs, on collider as well as detector R\&D issues. The early LHC input will therefore be crucial also from the economical point of view. However, many detailed studies are still missing in this context and should be addressed in the near future.

\subsection{Summary and conclusions of WG3}

The energy imbalance in a plane perpendicular to the beam direction is called Missing Transverse Energy (MET). MET plays a crucial role for studying Standard Model physics as well as for detecting physics beyond the SM, for instance, for detecting the stable lightest supersymmetric particles in SUSY models with R-parity conservation.

Concerning the experimental performance, it is expected that the purely calorimetric MET measurement in ATLAS is more precise than in CMS due to the better energy resolution and the higher longitudinal energy containment in the ATLAS hadronic calorimeter. In CMS, two methods that take into account other subdetectors have been developed to improve the purely calorimeter-based MET: the Track-Corrected MET and the Particle-Flow MET. It is expected that these methods improve the MET resolution up to a factor of about two. In particular the conceptual differences between the three approaches will guide and lead to a rather quick experimental determination of the MET. The $Z+$ jets process, where the $Z$ decays to electrons and muons, is ideally suited to study the MET performance since it is almost background-free and the two leptons can be measured with excellent precision. This process can also directly be used for data-driven background estimations for processes involving MET. The $Z \rightarrow \tau^{+} \tau^{-}$process can be used to determine the MET scale to about $8 \%$ accuracy. A global good understanding of the detector, however, will be mandatory to infer on event-by-event basis the total missing transverse momentum accurately from the measured transverse momentum.

Although it is expected that even for a moderate assumed luminosity of $1 \mathrm{fb}^{-1}$ squarks and gluinos with masses of order $1.3 \mathrm{TeV}$ should be discovered for a very broad range of R-parity conserving SUSY models, one should be aware of a few experimental caveats: a SUSY signal has no distinctive features, such as mass peaks which would separate it from possible SUSY background processes. Only SM backgrounds may be easily separated via appropriate cuts. Therefore one has to deal with counting experiments where an accurate prediction of the possible background processes may be mandatory for a correct interpretation. Excellent prospects exist for mass measurements, in particular in measuring mass differences. Several methods have been proposed to reconstruct the masses of particles in events including invisible particles. The absolute mass scale that is of most interest for planning future experiments, however, is particularly challenging to determine in the experiments.

Invariant mass endpoints or polynomial constraints of the observed four-momenta lead to a good determination of masses in sufficiently long cascade decays. The only known method working for any decay topology is the recently renewed method of measuring the transverse mass $m_{T}$. This method has originally been performed to measure $m_{W}$ precisely. However, in cases with missing energy complications arise. For instance, due to the expected non-negligible mass of new invisible particles, the transverse mass is no longer observable. This problem may be overcome by calculating the distributions for some hypothetical values. It is expected that kinks are featured 
exactly at the point where the hypothetical mass equals the true invisible mass. Generalization to more complex topologies might lead to a determination of all unknown masses. Whether ot not this method can actually successfully be employed in experiments remains to be seen.

Another important topic concerns the spin determination of new particles. Reconstructing different reference frames seems mandatory to reveal the underlying spin information. Invariant mass distributions of various visible final state particles and the determination of angular effects in the production processes give complementary information on the spin property. Exploiting further the spin property, as for instance, via analyzing the polarization of top quarks might be important for determining the mixing properties of the SUSY partners, i.e. the stop mixing angle. In this context a rather new set of observables turns out to be promising for the determination of the stop mixing angle: measuring ratios of different branching ratios in stop decays: accuracies at percent level in the determination of the stop mass and mixing angle might be achievable. Further studies of this promising set of observables including precise simulations of background processes and detector effects are desirable.

Signals with missing energy consist, for instance, of the lightest stable SUSY particle (LSP). The LSP is a promising cold dark matter candidate. Entering a new precision era with the results from WMAP, precise predictions of the respective relic density and its dark matter contributions in new physics models are required. Only for a rather restricted number of topologies, LHC data alone can provide a sufficiently accurate prediction of the DM candidate. It is expected that precision results from a linear collider in combination with LHC results are needed to finally determine the question of dark matter and achieve consistency with the current experimental precision bounds.

Results from a linear collider will also be mandatory to reveal the underlying physics, to determine the underlying SUSY parameters in a model-independent way and to determine the properties of the new particles. These results have been obtained by several physics analyses and are also confirmed by multi-parameter fits implemented in several numerical codes.

Two technologies for a linear collider are under discussion: the ILC and the CLIC concept. The ILC, with a first energy stage of $\sqrt{s}=500 \mathrm{GeV}$ is already on a mature design stage, feasible and under further responsibility of the global GDE. The feasibility of the CLIC concept has still to be demonstrated in the future, but has the potential to be applicable up to the multi-TeV range. Results from early LHC data may be important for specifying the detector requirements of the LC, for instance in cases where hints for CP-violation may be found in new physics. Early LHC results may also be decisive for defining the required energy stages of the later phase of the LC. Even in worst case scenarios, i.e. observing nothing or only a SM-like Higgs, precision measurements at the LC at a first energy stage of the top quark threshold or even only at the Z-pole are scientifically well motivated and have a large potential for revealing effects of new physics. Since the LC has its great potential in discoveries via precision measurements, future design considerations of a LC may therefore take into account a possible technical impact of design issues on the subsequent precision potential. A reliable and economic prediction of the required high energy scale of a future $\mathrm{TeV}$ machine may only be achievable if LHC data are interpreted in combination with precision results from a LC in its first energy stage of $\sqrt{s}=500 \mathrm{GeV}$. Therefore a staged approach of a future LC, providing precision physics from the Z-pole up to the new physics scale, seems to be reasonable and highly desirable. 


\title{
4 WG4: Other new physics signatures
}

\author{
A. De Roeck, T. Han, J.L. Hewett, S. Riemann (convenors) \\ G. Azuelos, M. Carena, K.F. Chen, H. Dreiner, A. Giammanco, S. Gopalakrishna, W.S. Hou, \\ G. Isidori, J. Kalinowski, E. Kou, D. Milstead, T.G. Rizzo, S. Sultansoy, B. Webber
}

In this section we examine the ability of the Tevatron, LHC, and future facilities to discover and interpret new phenomena that does not involve a missing energy signature. We discuss the production of a new $\mathrm{Z}$ boson as well as other states that lead to a leptonic resonance, the fourth family, testing seasaw mechanisms at colliders, exotic signatures of new physics, black hole production, and the impact of high precision flavor physics.

\subsection{Introduction and scenarios}

A strategy of planning for the future is to consider a wide variety of possible new phenomena and discern which types of facilities would be best to first discover new physics and then to elucidate its properties. This approach will hopefully prepare us for a broad set of possible signatures and for the surprises that Nature undoubtedly has in store, whatever they may be.

The goal of the investigations in this section was to explore signatures for new physics that do not involve missing energy or are not related to the production of a Higgs-like boson. These include:

- Leptonic and other resonances

- Multi gauge boson resonances

- Leptoquark type signatures

- Fourth generation and exotic quark production

- TeV scale gravity signatures

- New signatures such as heavy stable charged particles.

- New physics related to flavor physics

These scenarios were covered in review talks or in discussion sessions during the workshop; a number of these topics are discussed in this report. A benchmark signature for new physics is a leptonic resonance from, e.g., a new gauge boson $\left(Z^{\prime}\right)$ and is discussed here in detail. This signal is illustrative of what can be learned at the LHC, but also of what will remain elusive, and how we can advance our knowledge with future machines. Another detailed study was performed for the presence of a 4th generation of quarks and leptons. Here, the LHC may discover or exclude this scenario in the early stages of operation, with implications for a future collider. We next report on the possibility of distinguishing at colliders the various approaches of implementing the seasaw mechanism in the neutrino sector. We then discuss the ability of LHC detectors, with implications for future experiments, to observe exotic signatures of new physics such as stable charged particles, stopped particles, and non-pointing photons. The production of micro black holes is a possibility in theories with visible extra spatial dimensions at the Terascale. The signatures for this reaction are striking, although there are numerous associated theoretical uncertainties which could be pinned down by experimental measurements. Lastly, we discuss the implications of ultra-high precision 

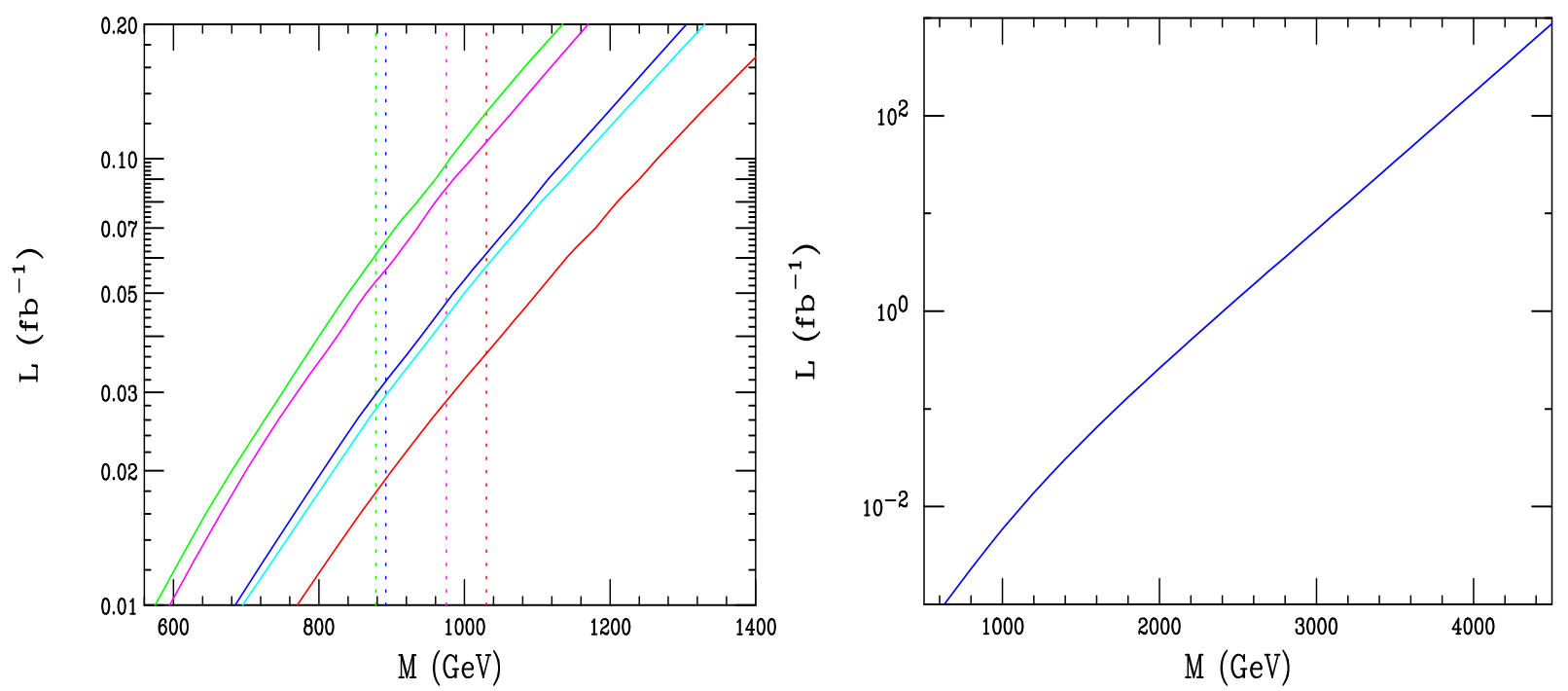

Fig. 4.1: $5 \sigma$ discovery reaches for (left) $Z^{\prime}$ in the $\psi$ (green), $\chi$ (cyan), $\eta$ (magenta), Left-Right Model(blue) and SSM(red) cases and for (right) $W^{\prime}$ (SSM case)at the $10 \mathrm{TeV}$ LHC as a function of the integrated luminosity. The vertical dotted lines are the present Tevatron bounds for the corresponding color-coded models.

measurements in the heavy quark sector and the role such measurements play in the discovery and elucidation of new interactions.

\section{2 $Z^{\prime}$ production at future colliders}

Once the LHC turns on, one of the cleanest potential signals for new physics beyond the Standard Model (SM) will be a $Z^{\prime}$-like resonance in the dilepton/Drell-Yan channel. Such an object is predicted to exist in an ever-widening set of new physics scenarios [239-246]. In some cases the $Z^{\prime}$-like object is also accompanied by an analogous $W^{\prime}$-like state whose presence will help us to identify the nature of the underlying physics. Present constraints from the Tevatron, employing the standard-candle Sequential Standard Model (SSM) scenario wherein the $Z^{\prime}$ and $W^{\prime}$ are just heavier versions of the usual SM states, imply that the masses of such particles typically lie above $\sim 1 \mathrm{TeV}$. If their couplings happen to be somewhat weaker than the typical electroweak strength, far lighter $Z^{\prime} / W^{\prime}$-like states may exist which could have been missed at the Tevatron. The LHC, even running at $10 \mathrm{TeV}$ and with an initial integrated luminosity of $100-200 \mathrm{pb}^{-1}$ has a chance to make a $Z^{\prime}$ or $W^{\prime}$ discovery as can be seen in Fig.4.1.

Resonances that are very weakly coupled to the SM fields are present in many models; in many cases their SM couplings are generated only via mixing with one or more of the SM gauge bosons. In this case, the state will be rather narrow and may be hard to find due to issues of mass resolution. The LHC, however, can go fairly deep into the small coupling parameter space provided sufficient luminosity is available. For example, a $1 \mathrm{TeV} \mathrm{SSM}$-like $Z^{\prime}$ with a coupling $\sim 1 / 20$ of the usual SM strength should be easily visible above the SM background at the $14 \mathrm{TeV}$ LHC with a luminosity of $100 \mathrm{fb}^{-1}$.

Once a $Z^{\prime}$ state is discovered we will want to discern its properties. Our goal will be to 
try to identify the underlying theoretical structure from which it arose. In many models the new resonance is accompanied by a number of other new particles. Here we will try to address what we can learn from the new resonance itself.

The first thing to determine is its lineshape, i.e., its mass and width and whether or not it is a (single) Breit-Wigner (BW) resonance. Unparticle resonances provide a good example of a nonBreit-Wigner lineshape that we may hope to distinguish from something more conventional [247]. The shape of this un-resonance is controlled by the unparticle mass and coupling strength as well as the effective anomalous dimension. The unusual lineshape of this un-resonance can be seen in the left panel of Fig.4.2 for various values of the parameters. Detector resolution can be of significant importance in performing the detailed measurements necessary to identify this non-BW structure. For some values of the parameters it is clear that the non-Breit-Wigner shape will be apparent although a detailed study has yet to be performed to determine the parameter ranges for which this differentiation can be performed.

Another possibility to consider is that there are 2 or more (almost)degenerate resonances which may interfere with each other, as well as the SM exchanges, thus distorting the expected line-shape. This can happen, e.g., in the case of string resonances or in extra-dimensional models where KK excitations of both the $\gamma$ and $Z$ can appear. This scenario also needs further study.

Next, we will want to determine the particle's spin; as is well-known, this can be acheived by examining the angular distribution of the final state leptons. While only $\sim 10$ clean events will be necessary to discover a $Z^{\prime}$-like state, hundreds of events will be needed to perform a measurement of the angular distribution. This implies that the 'reach' for spin determinations is significantly lower than for discovery and likely to be less than $\sim 3 \mathrm{TeV}$ for the SSM $Z^{\prime}$ with design machine parameters. In this case, if the LHC finds evidence for a Z'-like object in the data, an LHC luminosity upgrade will be extremely beneficial for a more detailed understanding of this new object.

After the lineshape and particle spin are determined we will want to know the couplings of this new state to the fields of the SM. (Recall that if it decays to $\gamma \gamma$ it cannot be spin-1.) This subject has been widely discussed in the literature [239-246,248] so we will be brief here. In the simplest case where the couplings are generation-independent and isospin invariant, there are 5 independent parameters to determine, one corresponding to each of the basic SM fields. Traditionally, one combines measurements of the resonance production cross section and width, the forward-backward asymmetry, $A_{F B}$, of the dilepton pair both on and off the resonance as well as the dilepton rapidity distribution to restrict the various couplings. Two important observations are: (a) this requires a rather large amount of integrated luminosity, $\sim 100 \mathrm{fb}^{-1}$, even for a relatively light $1.2 \mathrm{TeV} Z^{\prime}$ and (b) there are not enough observables in this list to make a unique determination of the 5 coupling parameters. There are, however, further measurements [239-246] that may be helpful when high luminosities are available: $(i)$ associated $Z^{\prime} V$ production with $V=\gamma, Z, W^{ \pm}$ (ii) rare $Z^{\prime}$ decay branching fractions to $f \bar{f} V,\left(V=Z, W^{ \pm}, f=\ell \nu\right)$, (iii) polarization of $\tau$ 's from $Z^{\prime}$ decay, (iv) $Z^{\prime} \rightarrow W^{+} W^{-}, Z h, b \bar{b}$ and $t \bar{t}$. Most of these have not yet been studied in any detail for the LHC.

Clearly, a future linear collider will be the ideal machine to study the properties of a Z'like object, in particular to accurately measure its mass, width, couplings and spin properties. This is particularly true if the new object is within the collider's energy range and can be produced directly. In addition a high energy electron-proton collider, such as proposed in the LHeC study [249], with polarized lepton beams, can yield additional information for the determination 

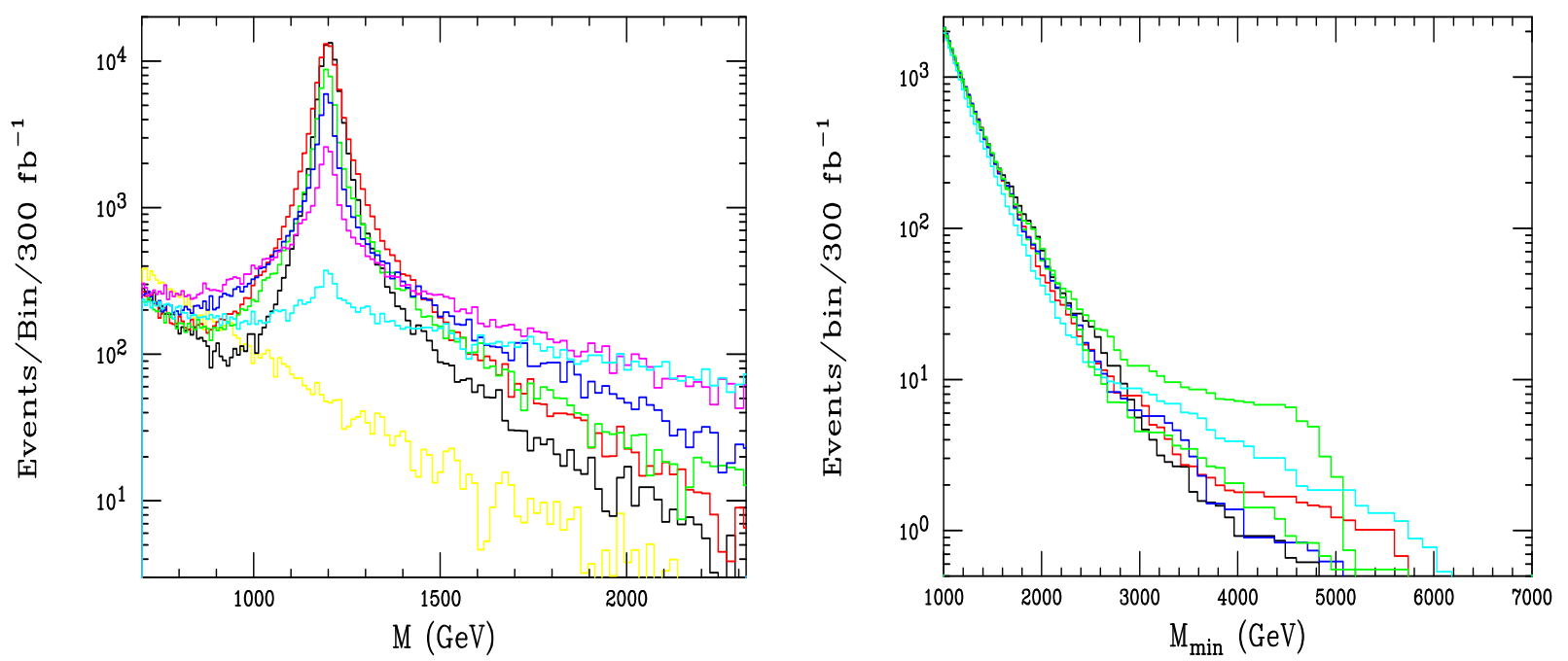

Fig. 4.2: (Left) Unparticle lineshapes (colored) in comparison to a SSM $Z^{\prime}$ (black) with a mass of $1.2 \mathrm{TeV}$ at the $14 \mathrm{TeV}$ LHC; a $1 \%$ mass resolution has been assumed. The yellow histogram corresponds to the SM expectation [240]. (Right) Dilepton event rate expected above a minimum invariant mass for the SM(black), the SSM with a $6 \mathrm{TeV} Z^{\prime}$ (red), a photon/ $Z$ gauge $\mathrm{KK}$ state with a mass of $6 \mathrm{TeV}$ (cyan), a Randall-Sundrum graviton with a $5 \mathrm{TeV}$ mass(blue) and $k / M_{p l}=0.04$ and a $5 \mathrm{TeV}$ (the $5 \sigma$ discovery reach in this case) $R$-parity violating sneutrino(green) with electromagnetic strength couplings to both quarks and leptons. An additional (lower) green histogram is also present for the spin-0 $R$-parity violating sneutrino case assuming a resonance mass of $6 \mathrm{TeV}$. Detector smearing has been included and the reader should remember Poisson statistics.

of the couplings [250].

In addition, one could ask whether a new $Z^{\prime}$-like state may be discovered indirectly at the LHC if its mass is too large to produce an observable resonance [251]. As is well known, high energy $e^{+} e^{-}$colliders can make precise measurements of the various $e^{+} e^{-} \rightarrow f \bar{f}$ processes and look for deviations due to the exchange of high mass states. Furthermore, it is possible that $e^{+} e^{-} \rightarrow f \bar{f}$ measurements made at different $\sqrt{s}$ values can be also be combined to determine the mass of the new resonance itself, provided the mass is not too far above the range of $\sqrt{s}$ values at which the data were taken [252]. At the LHC we have access to the entire Drell-Yan dilepton mass distribution; can we use it to see heavier states indirectly and determine their masses? Note that here we are not looking for contact interaction effects, but are trying to determine the mass of an essentially invisible object. In Ref. [251] an analysis was performed to address this issue. Specifically, it sought indirect evidence for states with masses $1 \mathrm{TeV}$ beyond the $14 \mathrm{TeV} 5 \sigma$ discovery reach assuming an integrated luminosity of $300 \mathrm{fb}^{-1}$. States with spin- 0,1 and 2 were considered. Based on this study it appears that, except for the very special case of degenerate $Z / \gamma$ KK resonances (where there is strong destructive interference with the SM exchanges far below the resonance), the answer to this question appears to be 'no'. Results of this preliminary analysis are shown in the right panel of Fig. 4.2. Evidently, the rapid fall-off of the parton densities at the required high- $\mathrm{X}$ values prevent us from gathering sufficient statistics to perform the same procedure as in the $e^{+} e^{-}$ case. If we see any deviation in the spectrum we will already see the resonance itself.

If a $W^{\prime}$ accompanies the $Z^{\prime}$ and decays into $\ell \nu$, the most important thing to determine is the 
helicity of its couplings to the SM fermions as these are usually chiral. This simple measurement broadly splits all possible models into 2 classes depending upon whether these couplings are LH or RH. This measurement cannot be performed on the 'peak' of the transverse mass distribution since there is no sensitivity 'on-resonance' as only pure $W^{\prime}$ exchange is being sampled. However, there is a significant effect in the transverse mass region below the peak as in the case of RH couplings there is no interference with the SM $W$ contribution while there is for LH couplings. This is shown explicitly in Fig. 4.3 from Ref. [253].
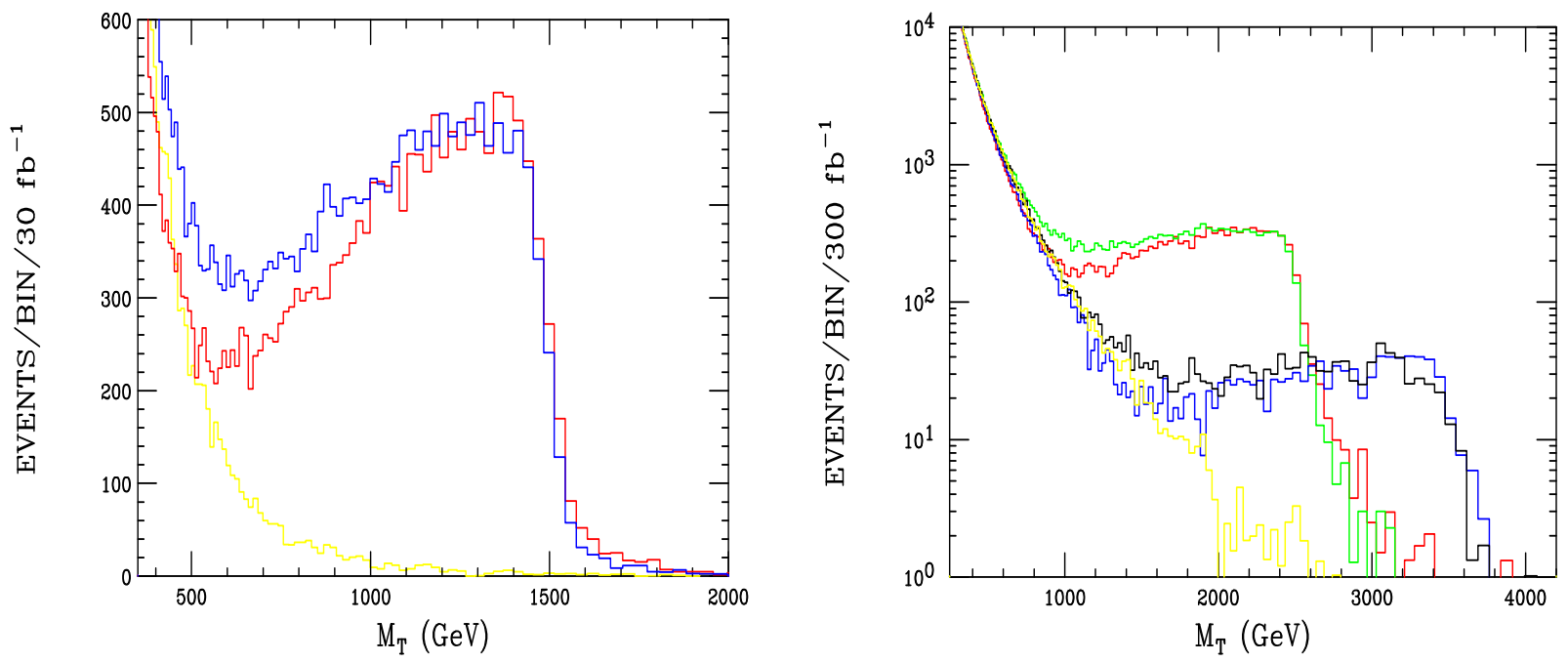

Fig. 4.3: $W^{\prime}$ transverse mass distributions for (Left) $M_{W^{\prime}}=1.5 \mathrm{TeV}$ and for (Right) $M_{W^{\prime}}=2.5$ and 3.5 $\mathrm{TeV}$ [246]. The upper(lower) histogram in each case corresponds to right(left)-handed couplings to the SM fields. A $2 \% M_{T}$ smearing has been included in these results.

\subsection{4th Generation of Fermions}

The addition of a fourth generation of chiral fermions (4SM) to the SM has long been investigated as one of the simplest extensions of the SM. At the advent of the LHC start-up, it is pertinent to reconsider the physics potential of the 4SM. The Tevatron has excluded the $t^{\prime}$ quark of mass less than around $300 \mathrm{GeV}$. This limit can be further pushed up to around $500 \mathrm{GeV}$ during the first year of LHC data taking. In this report, we review the impacts of the forthcoming LHC data on the theory as well as on the prospects of a future Linear Collider experiment.

\subsubsection{4th generation: is it suggested by the present data?}

CDF sets a $311 \mathrm{GeV}$ mass limit [254] at 95\% CL for a $t^{\prime}$ decaying into a light quark plus a $W$ with a reported excess in a mass region centered at $450 \mathrm{GeV}$. At present there is an ongoing search for a $t^{\prime}$ quark at $\mathrm{D} \varnothing$ which may help clarify this situation. The $b^{\prime}$ quark has been searched for in the $b Z$ [255] channel. This decay occurs via a loop and may compete with the tree-level process $b^{\prime} \rightarrow t W$, with the latter mode being more difficult to reconstruct (due to combinatorials with up to 10 jets). 
In the $4 \mathrm{SM}$, the $3 \times 3 \mathrm{CKM}$ matrix is only part of the full matrix, thus the unitarity of this $3 \times 3$ portion could be broken. It is often considered that the $3 \times 3 \mathrm{CKM}$ matrix is unitary at high precision, however, the 3rd row is not determined precisely by tree-level processes. Constraints on the $4 \times 4 \mathrm{CKM}$ matrix may be obtained by computing the $t^{\prime} / b^{\prime}$ contributions to FCNC and electroweak observables. Such studies have been recently updated [256-258]. In addition, recent Tevatron data for the CP asymmetry in $B_{s}$ mixing shows a 2-3 $\sigma$ deviation from the 3SM [259], which can be explained by the two new CP violating phases in the 4SM.

In addition, it has recently been emphasized that the electroweak oblique parameters do not exclude the 4SM [256-258, 260]. It is usually considered that the large contribution from the 4th generation quarks to the $S$ parameter creates a tension with data. However, if there is an extra contribution to the $T$ parameter (e.g. from non-degeneracy of $t^{\prime}$ and $b^{\prime}$ or the 4 th generation leptons), one can easily accomodate the 4SM within the experimental allowed ranges.

\subsubsection{Impact of a 4th generation quark around $500 \mathrm{GeV}$}

Here, we consider the scenario where the 4th generation quark has a mass around $500 \mathrm{GeV}$. We focus on this mass range not only because it is the early LHC early discovery reach but also because this corresponds to the perturbative unitarity limit for the 4th generation [261] and thus it provides an important theoretical benchmark. A mass scale heavier than this would imply: i) we are entering the strongly coupled regime and thus we would expect some consequences such as a condensate of the heavy fermions, and ii) new physics would need to be introduced in order to satisfy the unitarity limit.

Such a heavy $t^{\prime}$ impacts the Higgs mass limits, in particular the bounds from stability/triviality. This occurs due to the $t^{\prime}$ Yukawa coupling contribution to the loop correction to the Higgs quartic coupling, $\lambda$. The solution to the renormalization group equation (RGE) for this quartic coupling gives the Higgs mass in terms of the cut-off scale $\Lambda$. When $\lambda$ becomes negative, the Higgs potential no longer has a stable minima. This limit leads to a lower bound for the Higgs mass (the so-called stability limit). For the $3 \mathrm{SM}$ case, the obtained Higgs mass limit is $M_{H}>130(70) \mathrm{GeV}$ for $\Lambda \simeq M_{\text {Planck }}(1 \mathrm{TeV})$ [262]. The RGE shows that the large 4th generation Yukawa couplings increase this lower bound. On the other hand, as the Higgs mass increases, there is a point where the quark Yukawa term exactly cancels the other contributions. This leads to an upper bound on the Higgs mass (the so-called triviality limit). The RGE shows that this fixed point yields the upper bound of $m_{H}=500-800(200) \mathrm{GeV}$ for $\Lambda=1\left(M_{\text {Planck }}\right) \mathrm{TeV}$ [263] in the 3SM, while the larger value of the fourth generation Yukawas dramatically lowers this upper bound. In fact, it has been shown in [260] that even for $m_{t^{\prime}}=m_{b^{\prime}}=260 \mathrm{GeV}$, the Higgs mass is constrained to the range $200<M_{H}<470 \mathrm{GeV}$ for $\Lambda=1 \mathrm{TeV}$ and most importantly, the famous chimney (i.e. for $130<M_{H}<200$, the 3 SM is valid up to the Planck scale) is closed out. In summary, i) the Higgs mass lower limit (the stability bound) increases as the 4th generation quark masses increase, and ii) the 4SM ceases to be valid for $\Lambda \gtrsim 10^{\text {a few }} \mathrm{TeV}$ if a 4 th generation quark heavier than the current Tevatron limit is discovered. It has been emphasized that this situation does not change even if we extend the Higgs sector [264].

It has also been shown [265] that electroweak baryogenesis occurs at sufficient rates to pro-

duce the observed matter anti-matter asymmetry in the case where the 4SM is further extended by SUSY (4MSSM), provided that the 4th generation is fairly heavy and its scalar partners have 
similar mass. We discuss a potential discovery of such scenario at the LHC and future colliders below.

\subsubsection{What do we expect during the early years of LHC?}

At the LHC, the cross section for heavy quark production is large and allows us to reach the unitarity mass limit with a few $100 \mathrm{pb}^{-1}$. Detection of the $t^{\prime}$ seems to be easy. The dominant channel $t^{\prime} \rightarrow b W$ has been studied in detail using the same strategy as for observing the top quark. The $b^{\prime}$ search is more involved as the decay $b^{\prime} \rightarrow t W \rightarrow b W W$ leads to a soft $W^{\prime}$ 's. The recent study estimates that the $t^{\prime}$ quark with mass $m_{t^{\prime}}=450 \mathrm{GeV}$ can be discovered at $5 \sigma$ level with the first year of data taking $\left(100 \mathrm{pb}^{-1}\right)$ [266]. In this workshop, CMS also reported that the $b^{\prime}$ with mass $m_{b^{\prime}} \leq 300 \mathrm{GeV}$ can be discovered at the $7.5 \sigma$ level with the same amount of data. On the contrary, leptons are produced by the Drell Yan process and requires $\gtrsim 10 \mathrm{fb}^{-1}$ for a meaningful search.

It is well-known that the addition of two heavy quarks increases the coupling of the Higgs to two gluons by a factor of 9 for $m_{H}=125 \mathrm{GeV}$ and of 5 for $m_{H}=500 \mathrm{GeV}$ [267]. Therefore, naively speaking, to get a $5 \sigma$ discovery, one needs a factor of 81 less luminosity than for the 3SM at 14 $\mathrm{TeV}$. Thus, the first year of data would allow for a Higgs discovery in the $\sim 350-500 \mathrm{GeV}$ region. It is interesting to note that given the factor of 9 enhancement for the gluon-gluon process, one can already say that the mass region $135-200 \mathrm{GeV}$ is excluded by Tevatron in the 4SM. The search for a light Higgs remains difficult at the LHC in spite of the enhancement factor for the cross section since $\operatorname{Br}(H \rightarrow \gamma \gamma)$ is reduced by a factor of $\sim 10$. The consequences are that the most efficient channel will be $H \rightarrow Z Z^{*}$. A detailed analysis is needed to provide a more quantitative answer but following [260], one expects that a few $\mathrm{fb}^{-1}$ would be needed.

Within the SUSY+baryogenesis scenario, squarks and heavy quarks are being produced with similar rates (except for the $\beta$ factor for scalar production) which may result in a rich but confusing situation. For instance $b^{\prime} \rightarrow t W$ final states may overlap with a squark $b^{\prime}$ giving a top quark and a chargino.

\subsubsection{What is the role of the future linear collider?}

The mass limit for heavy quarks set by unitarity calls for a LC reaching a centre of mass energy of $\sim 1 \mathrm{TeV}$. Very precise masses and couplings of the new heavy fermions can be measured using polarized beams and constrained reconstruction. Branching fractions for Higgs decays into the 4th leptons, if kinematically allowed, would be well measured at a LC. One could also observe stable or long lived neutrinos through neutrino counting.

Within the 4MSSM there could be a Higgs lighter than $135 \mathrm{GeV}$ giving access to the fermionic decay modes with incomparable accuracies. Also the precise measurement of the branching ratio of $H \rightarrow g g$ would be crucial to determine the Yukawa couplings of the heavy quarks. A consideration of the successful baryogenesis through 4MSSM requires mass degeneracy of the heavy quarks and squarks. In such a situation, the LC becomes most powerful: threshold scans would allow to disentangle the various degenerate quark and squark states. 


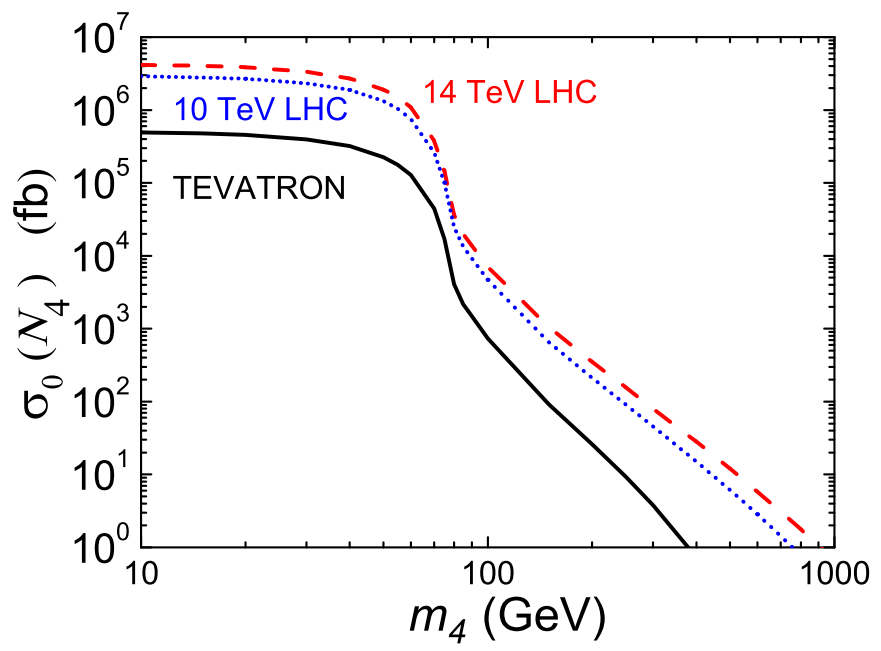

Fig. 4.4: The bare cross section $\sigma_{0}\left(N_{4}\right)$ versus mass of heavy Majorana neutrino $m_{4}$ for the Tevatron ( $p \bar{p}$ at $1.96 \mathrm{TeV}$, solid curve) and the LHC ( $p p$ at 10 and $14 \mathrm{TeV}$, dotted and dashed curves, respectively) [277].

\subsection{Testing Seesaw mechanisms at the LHC}

The neutrino mass and flavor oscillations are arguably the first indication for physics beyond the Standard Model (SM). The smallness of the neutrino mass is attributed to a large energy scale of new physics $\Lambda$, via the "Seesaw" relation $m_{\nu} \sim y^{2} v^{2} / \Lambda$ where $y$ is a Yukawa coupling. If $\Lambda$ is near the Terascale, one would have the hope to test the seesaw mechanism by searching for lepton-number violating signals.

\subsubsection{Type I Seesaw [268-272]: Heavy Majorana Neutrinos}

Searching for heavy Majorana neutrinos at hadron colliders have long been considered by many authors [273], however the interest for the LHC has been lately renewed [274-276]. We calculate the exact process, but it turns out to be an excellent approximation to parameterize the cross section as

$$
\sigma\left(p p \rightarrow \ell_{1}^{ \pm} \ell_{2}^{ \pm} W^{\mp}\right) \approx\left(2-\delta_{\ell_{1} \ell_{2}}\right) \sigma\left(p p \rightarrow \ell_{1}^{ \pm} N_{4}\right) \operatorname{Br}\left(N_{4} \rightarrow \ell_{2}^{ \pm} W^{\mp}\right) \equiv\left(2-\delta_{\ell_{1} \ell_{2}}\right) S_{\ell_{1} \ell_{2}} \sigma_{0}\left(N_{4}\right)
$$

where $\sigma_{0}\left(N_{4}\right)$, called the "bare cross section", is only dependent on the mass of the heavy neutrino and is independent of all the mixing parameters when the heavy neutrino decay width is narrow. We calculate the exact cross section for the dilepton production and use the definition Eq. (4.1) to find the bare cross sections $\sigma_{0}\left(N_{4}\right)$. These are shown in Fig. 4.4 at the Tevatron and LHC energies versus the mass of the heavy Majorana neutrino, where we have calculated the cross sections at both 10 and $14 \mathrm{TeV}$ c.m. energies. The production rate is increased at the higher energy by a factor of $1.5,2.0,2.5$ for $m_{4}=100,550$ and $1000 \mathrm{GeV}$, respectively. 

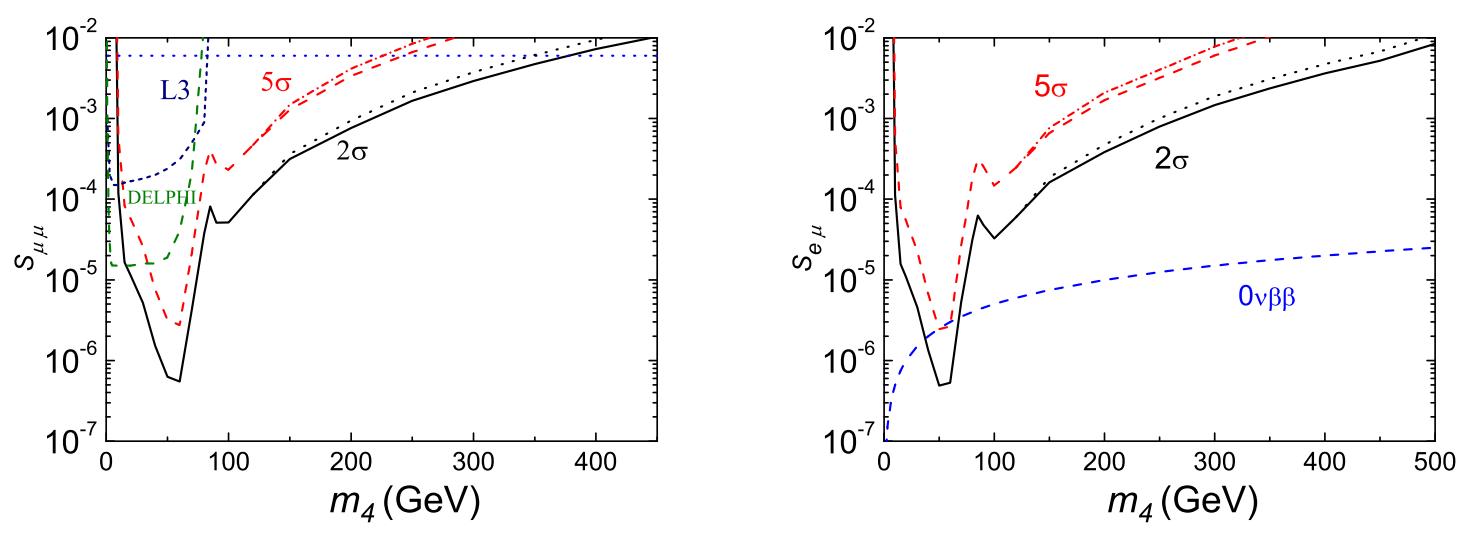

Fig. 4.5: (a) Left: $2 \sigma$ and $5 \sigma$ sensitivity for $S_{\mu \mu}$ versus $m_{4}$ at the LHC with $100 \mathrm{fb}^{-1}$ integrated luminosity; (b) right: same as (a) but for $S_{e \mu}$ (both plots taken from Ref. [277]). The solid and dashed (dotted and dash dotted) curves correspond to limits with the exclusion (inclusion) of the Higgs decay channel for $m_{H}=120$ $\mathrm{GeV}$. The horizontal dotted line corresponds to the constraint on $S_{\mu \mu} \simeq\left|V_{\mu 4}\right|^{2}<6 \times 10^{-3}$ from precision EW measurements.

The flavor information of the final state leptons is parameterized by

$$
S_{\ell_{1} \ell_{2}}=\frac{\left|V_{\ell_{1} 4} V_{\ell_{2} 4}\right|^{2}}{\sum_{\ell=e}^{\tau}\left|V_{\ell 4}\right|^{2}} .
$$

In general the two final state charged leptons can be of any flavor combination, namely,

$$
e^{ \pm} e^{ \pm}, e^{ \pm} \mu^{ \pm}, \quad e^{ \pm} \tau^{ \pm}, \mu^{ \pm} \mu^{ \pm}, \mu^{ \pm} \tau^{ \pm} \text {and } \tau^{ \pm} \tau^{ \pm}
$$

The constraint from $0 \nu \beta \beta$ is very strong and makes it difficult to observe like-sign di-electrons $e^{ \pm} e^{ \pm}$. The events with $\tau$ leptons will be challenging to reconstruct experimentally. We will thus concentrate on clean dilepton channels of $\mu^{ \pm} \mu^{ \pm}$and $\mu^{ \pm} e^{ \pm}$.

In Fig. 4.5(a) and Fig. 4.5(b), we summarize the sensitivity for $S_{\mu \mu}$ and $S_{e \mu}$ versus $m_{4}$, respectively. The solid (dashed) curves correspond to $2 \sigma(5 \sigma)$ limits on $S_{\ell \ell^{\prime}}$ with the exclusion of the Higgs decay channel. The dotted (dash dotted) curves are similar but with the inclusion of the Higgs decay channel for $m_{H}=120 \mathrm{GeV}$. The horizontal dotted line corresponds to constraints on $\left|V_{\mu 4}\right|^{2}<6 \times 10^{-3}$ from precision EW measurements. In Fig. 4.5(b) the dashed line at the bottom corresponds to the limit from $0 \nu \beta \beta$.

We find that, at the Tevatron with $8 \mathrm{fb}^{-1}$ integrated luminosity, there could be $2 \sigma(5 \sigma)$ sensitivity for resonant production of a Majorana neutrino in the $\mu^{ \pm} \mu^{ \pm}$modes in the mass range of $\sim 10-180 \mathrm{GeV}(10-120 \mathrm{GeV})$. This reach can be extended to $\sim 10-375 \mathrm{GeV}(10-250 \mathrm{GeV})$ at the $\mathrm{LHC}$ of $14 \mathrm{TeV}$ with $100 \mathrm{fb}^{-1}$. The production cross section at the $\mathrm{LHC}$ of $10 \mathrm{TeV}$ is also presented for comparison. We study the $\mu^{ \pm} e^{ \pm}$modes as well and find that the signal could be large enough even taking into account the current bound from neutrinoless double-beta decay. However, it is believed that any signal of $N$ would indicate a more subtle mechanism beyond the simple Type I seesaw due to the otherwise naturally small mixing $V_{N \ell}^{2} \sim m_{\nu} / M_{N}$ between $N$ and the SM leptons. 

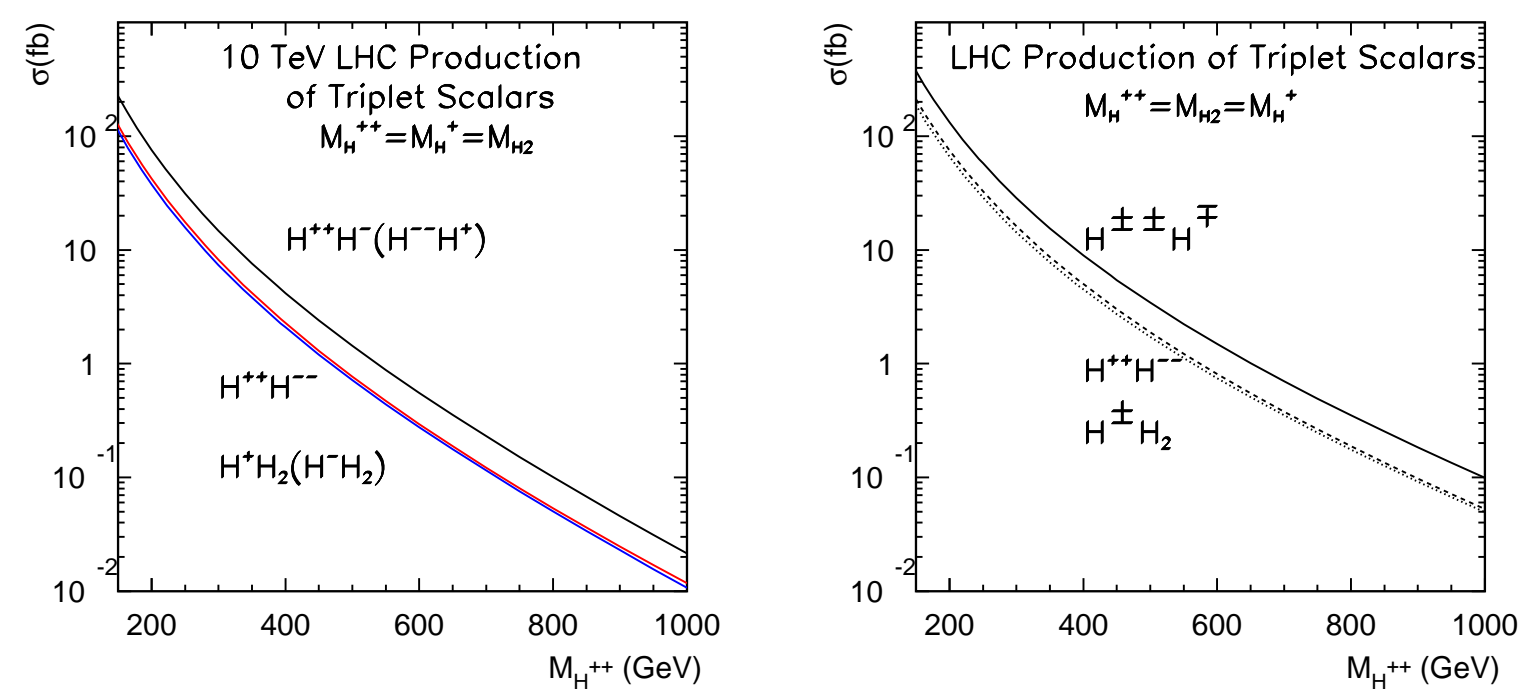

Fig. 4.6: Total production cross section at the LHC versus the heavy Higgs mass for (a) at $10 \mathrm{TeV}$, (b) at 14 $\mathrm{TeV}$ [284].

\subsubsection{Type II Seesaw [278-282]: Doubly Charged Higgs Bosons}

Several earlier studies of certain aspects of the Type II seesaw model at the LHC exist [283]. We find that in the optimistic scenarios, by identifying the flavor structure of the lepton number violating decays of the charged Higgs bosons at the LHC, one can establish the neutrino mass pattern of the Normal Hierarchy, Inverted Hierarchy or Quasi-Degenerate. We emphasize the crucial role of the singly charged Higgs boson decays. The associated pair production of $H^{ \pm \pm} H^{\mp}$ is essential to test the triplet nature of the Higgs field. The observation of either $H^{+} \rightarrow \tau^{+} \bar{\nu}$ or $H^{+} \rightarrow e^{+} \bar{\nu}$ will be particularly robust for the test since they are independent of the unknown Majorana phases. Combining with the doubly charged Higgs decay, for instance $H^{++} \rightarrow e^{+} \mu^{+}, e^{+} \tau^{+}, \mu^{+} \tau^{+}$, one will even be able to probe the Majorana phases.

The production cross sections for all three channels are shown in Fig. 4.6(a) for the LHC at $10 \mathrm{TeV}$, and in Fig. 4.6(b) at $14 \mathrm{TeV}$. With negligible SM backgrounds, the only limitation would be the event rate, that determines the statistical error for the BR measurements, $i$. $e$, a relative error $1 / \sqrt{N}$ if Gaussian statistics is applicable.

\subsubsection{Type III Seesaw [285]: Heavy Leptons}

In Fig. 4.7, we present the total production cross sections for the leading electroweak processes versus the heavy lepton mass $M_{T}$ at the LHC ( $p p$ at $\sqrt{s}=14 \mathrm{TeV}$ and $10 \mathrm{TeV}$ ). To view the generic feature, we have pulled out the effective couplings $\lambda^{2}$ in the plots, which is normalized to unity for the pair production, and to the Yukawa coupling squared for the single production.

The smoking gun is the production of lepton number violating same-sign dileptons plus four 


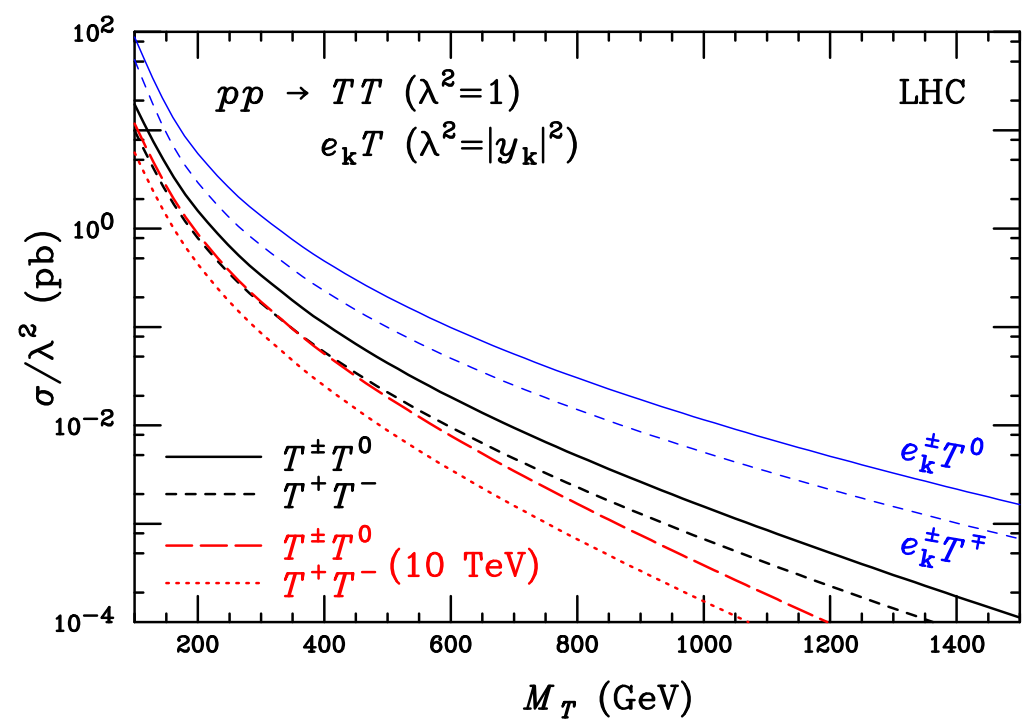

Fig. 4.7: Cross sections of single and pair productions of $T^{ \pm} / T^{0}$ as a function of its mass at the LHC (14 $\mathrm{TeV}$ and $10 \mathrm{TeV}$ ). The scaling constant $\lambda^{2}$ is 1 for $T T$, and $\left|y_{k}\right|^{2}$ for $e_{k} T$ [286].

jets without significant missing energy. Our analysis shows that via the unique channel,

$$
T^{0} T^{ \pm} \rightarrow\left(\ell^{ \pm} W^{\mp}\right)\left(\ell^{ \pm} Z / h\right), \text { or } \ell^{ \pm} \ell^{ \pm} W^{\mp} Z / h
$$

the heavy lepton can be searched for up to a mass of $200 \mathrm{GeV}$ at the Tevatron with $8 \mathrm{fb}^{-1}$, and up to 450 (700) $\mathrm{GeV}$ at the LHC of $14 \mathrm{TeV}$ C.M. energy with $10(100) \mathrm{fb}^{-1}$. The signal rate at the 10 $\mathrm{TeV}$ LHC is reduced by a factor of $60 \%-35 \%$ for a mass of $200-700 \mathrm{GeV}$.

In conclusion, if the scale for the neutrino mass generation is near the Terascale, it is possible to test the seesaw mechanism by searching for lepton-number violating signals at the LHC. The signatures are rather unique and clean. Higher integrated luminosity and energy would be beneficial for extending the search. A future $e^{+} e^{-}$linear collider would also be a sensitive probe of the Seesaw mechanism.

\subsection{New signatures and implications for detectors on new colliders}

Recently suggested theoretical scenarios predict the possibility of exotic signatures at the LHC, such as heavy stable charged particles, particles that may stop in the detector, non-pointing photons, monopoles etc. Present experiments such as ATLAS and CMS are often not specifically designed for these type of signatures and thus it has been an interesting exercise over the past few years to evaluate and design triggers and analysis methods to tackle the search for these new physics scenarios. Overall, and sometimes surprisingly, the detectors can handle these new physics signatures in general very well. Both ATLAS and CMS have been studying these new physics scenarios and a few examples are given in this section.

In some of these scenarios, heavy stable charged particles can be produced and a fraction of these can, via their energy loss in ionization and hadronic interactions, stop in the detector, sit 

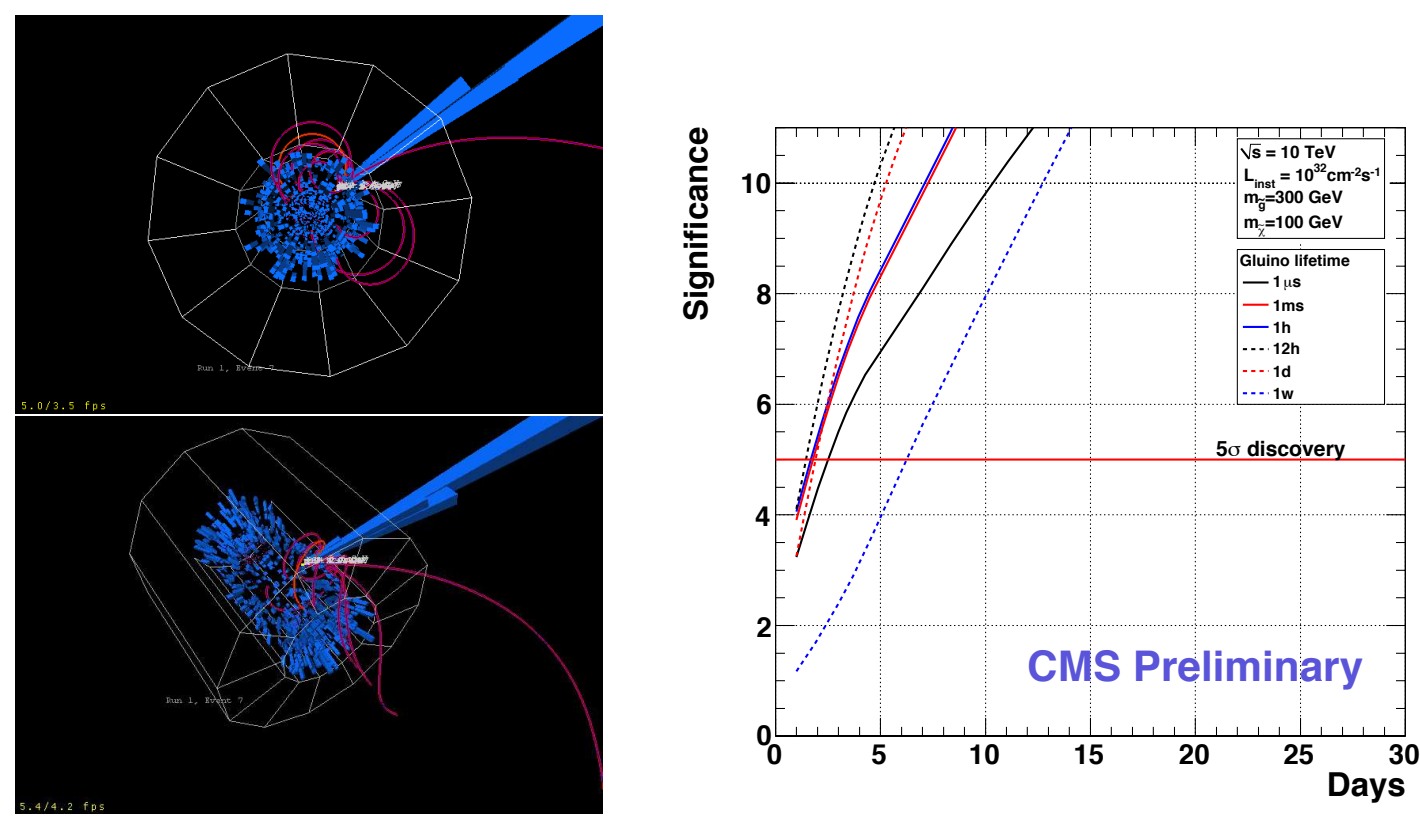

Fig. 4.8: (left) Simulated signal events in the detector, in the search for stopped gluinos; (right) significance that can be obtained with a luminosity of $10^{32} \mathrm{~cm}^{-2} \mathrm{~s}^{-1}$ for different gluino lifetimes (both plots taken from Ref. [287]).

there for a while (seconds, hours, days) and then decay. It is a challenge for the experiments to be ready for these signatures, in particular to trigger on these events. So far the experiments are found to be up to this challenge. CMS made a study for stopped R-hadrons driven by long lived gluinos. A good fraction, as much as a third of the produced R-hadrons can stop in the dense structure of CMS. After some time the R-hadron finally decays in a cluster of jets. Experimentally this will be most easily observable when such a decay happens during abort accelerator gaps or other empty bunch crossings, or when there is no beam in the machine all together. Backgrounds will mainly come from cosmics or noise in the detector, and these have already been studied with the CMS detector while waiting for LHC collisions. An example of a simulated signal event is shown in Fig. 4.8(left). CMS has designed a trigger that will detect energy clusters in the calorimeter during no-collision time. The sensitivity is shown in Fig. 4.8(right). Already with a few weeks of good luminosity $\left(10^{32} \mathrm{~cm}^{-2} \mathrm{~s}^{-1}\right) 5 \sigma$ significance discoveries can be made for gluinos with mass of 300 $\mathrm{GeV}$.

Other signatures include those where the particles pass through the detector, but since they move with a speed which is only a fraction of the speed of light, these will arrive late in the outer detectors. If the velocity $\beta$ of the particle is larger than 0.6 then a good fraction can be detected and reconstructed in CMS and ATLAS and the time delay can be measured, as shown in Fig. 4.9 (left). Also the energy loss by ionization in the tracker should be unusually high. Some of these signatures could well be visible with early data. For example Fig. 4.9(right) shows the luminosity needed for observing 3 events (in the anticipated absense of background) for different scenarios of new physics that lead to heavy stable charged particles. This includes KK taus, gluinos, stable stops and GMSB staus. The prospects are excellent! Now let's see what Nature really has in store for us... 

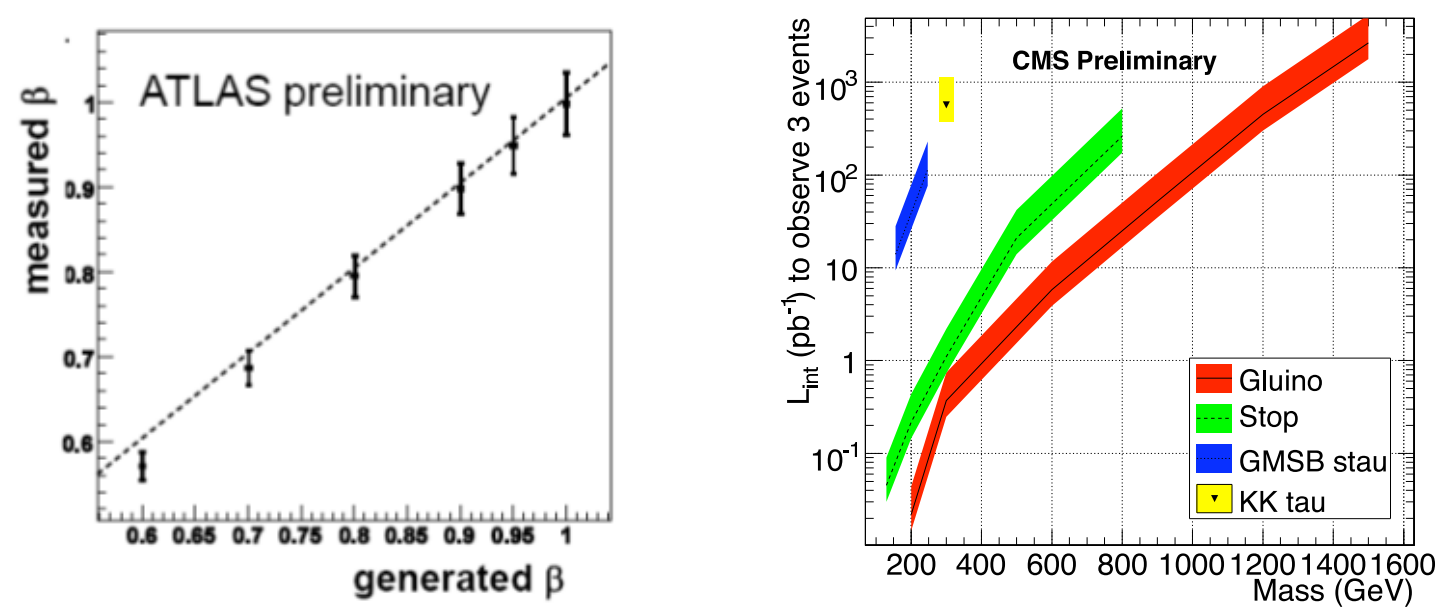

Fig. 4.9: (left) The timing of a heavy charged particle as measured in ATLAS compared with the true timing [2]; (right) Luminosity needed for a discovery requiring 3 events (for no background) for various heavy stable charged particles in CMS [288].

Other new signatures include monopoles, events with many displaced vertices (such as from Hidden Valley models), non-pointing photons (from e.g., GMSB models). ATLAS has demonstrated that the trigger for e.g., hidden valley models is under control for a good part of the expected phase space.

When such new particles are discovered, this will have a huge impact on the design of the detectors at future colliders or even the LHC detector upgrade. Precise time-of flight will become much more important, preferably at the 100 ps seconds or better. For the LHC itself it may be that one needs to keep data for more than one bunch crossing, ie longer than $25 \mathrm{~ns}$, in order to accept and measure particles with low $\beta$ values. If particles will move slower than roughly $\beta<0.6$ then they will reach the muon systems in the experiments at a time compatible with an interaction of the next bunch crossing. Good timing in the electromagnetic calorimeter in particular would allow also to detect cleanly non-pointing photons: the path crossed to reach a calorimeter cell will be larger when they come from a decay, compared to when these photons arrive in a straight line from the interaction point. Clearly any information from the LHC on the masses of the new particles will be important to define the next colliders minimum energy threshold.

\subsection{Black Holes}

In scenarios with large or warped extra dimensions, the higher-dimensional Planck mass $M_{D}$ could be as low as the TeV scale. For three or more extra dimensions, this is not excluded by astrophysical observations. Then gravity would be a much stronger force at short distances and black holes could be formed in multi-TeV particle collisions. For a recent review and references, see [289]. 


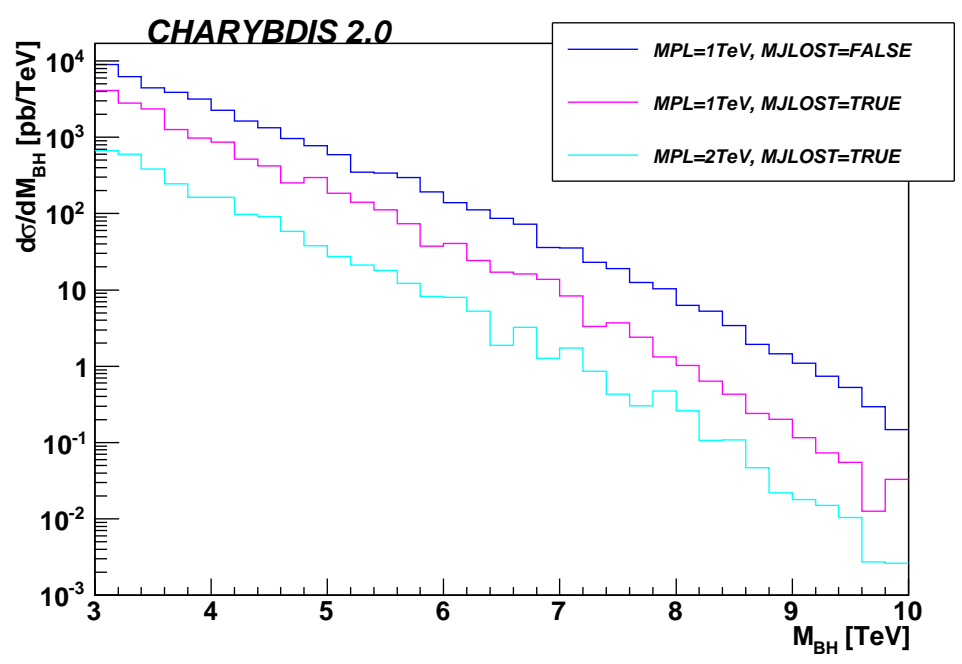

Fig. 4.10: Black hole cross section at LHC for $n=4$ extra dimensions [292].

At energies sufficiently far above $M_{D}$, it should be possible to treat black hole formation in particle collisions using general relativity extended to higher dimensions. Even in this classical approximation the dynamics of such a highly nonlinear process is not yet fully worked out. For a recent study in four dimensions, see [290]. For extra dimensions we have only lower limits on the impact parameter for formation and on the mass of the resulting black hole [291]. The collision energy that is not trapped in the black hole is emitted in gravitational radiation, which escapes detection. However, studies at zero impact parameter suggest that the losses of energy and angular momentum in this so-called balding process do not saturate the limits derived by Yoshino and Rychkov in [291].

The simulation program CHARYBDIS2 [292] includes a model for the balding process that satisfies the Yoshino-Rychkov bounds while being consistent with the results of other approaches at zero impact parameter. Typically $\sim 80 \%$ of the collision energy and angular momentum is trapped. The model is activated by setting the parameter MJLOST $=$. TRUE.. The production cross section for different values of the Planck scale MPL $=M_{D}$ and MJLOST options is shown in Fig. 4.10.

After formation, the black hole decays rapidly by Hawking evaporation. In CHARYBDIS2 and also in the program BLACKMAX [293,294], but not in earlier programs (including earlier versions of CHARYBDIS), angular momentum is taken into account. This affects the spectra and angular distributions of the emitted particles, and the relative abundances of different particle species. All Standard Model particles are assumed to be emitted "on the brane", i.e. they do not propagate into the extra dimensions. Their differential fluxes are given by

$$
\frac{d^{4} N_{\lambda}}{d \cos \theta d \phi d \omega d t}=\left.\left.\frac{1}{4 \pi} \sum_{j m} \frac{T_{j m}}{e^{\frac{\omega-m \Omega}{T}} \pm 1}\right|_{\lambda} S_{j m}(\theta, \phi)\right|^{2}
$$

where $\lambda, \omega, j$ and $m$ are the helicity, the energy and the total and azimuthal angular momentum quantum numbers of the emission, $T$ is the Hawking temperature, $\Omega$ is the horizon angular velocity, $T_{j m}$ is the coefficient for transmission from the horizon to infinity (the "greybody factor" modifying the purely thermal spectrum), and ${ }_{\lambda} S_{j m}$ is a (generalized) spheroidal harmonic function. The 
dependence of the thermal factor on $\omega-m \Omega$, which is just the energy in a frame co-rotating with the horizon, favour emissions with high values of $m$, which help the black hole to shed its angular momentum. The same dependence skews the spectrum toward higher energies, relative to the non-spinning case.

The spheroidal harmonic angular dependence leads to a distribution of higher-energy emissions concentrated around the equatorial plane of the black hole. Since the original angular momentum vector is approximately perpendicular to the beam directions, this implies a somewhat broadened rapidity distribution. Emission of particles with non-zero spin and appropriate helicity along the polar axes also enables the black hole to lose angular momentum and becomes more favourable at lower energies. Thus for example neutrinos and antineutrinos are preferentially emitted in the southern and northern hemispheres respectively. Similarly the decay angular distributions of emitted $\mathrm{W}$ bosons are strongly correlated with their polarization and hence also with the orientation of the black hole.

Owing to the high number of colour degrees of freedom, the Hawking emission is dominated by quarks and gluons. The polar emission mechanism mentioned above enhances the flux of vectors relative to fermions, and of fermions relative to scalars (including longitudinal vector bosons), compared to the fluxes from a non-rotating black hole. However, the shift in the spectra to higher energies means that the overall multiplicity of emitted particles is reduced.

One missing component of the existing simulations is Hawking emission of gravitons. This is because gravitons are emitted into the higher-dimensional bulk and the corresponding greybody factors and angular distribution functions for rotating black holes are unknown. ${ }^{4}$ For numbers of dimensions that are not too large, the relatively low number of graviton degrees of freedom probably make this a small effect, comparable with the uncertainties in the amount of gravitational radiation in the formation process.

As the Hawking radiation carries off energy and angular momentum, the black hole becomes lighter and loses its spin. On the average these processes occur in parallel, rather than as distinct spin-down and static evaporation phases. As the mass decreases the Hawking temperature rises, until the mass and/or temperature approach the Planck scale. At this stage the process leaves the classical realm and a quantum theory of gravity would be required to follow it further. The simulation programs include a variety of models for this Planck phase of black hole decay, ranging from a stable exotic remnant particle to a string-inspired option in which particles "boil off" at a fixed limiting temperature. See refs. [292-294] for details.

In conclusion, the production of higher-dimensional black holes in particle collisions remains a possibility worth exploring. The formation process is still not well understood and there are uncertainties in the fractions of the collision energy and angular momentum that would be trapped in the black hole. Once the initial conditions are established, the main phase of decay via Hawking radiation is under better control. The only missing component is bulk graviton emission. The terminal Planck-scale phase of decay is not understood but a variety of models are available and its contribution to the final state is probably not large.

The effects of black hole rotation during the Hawking emission phase are substantial: the spectra, angular distributions and relative abundances of particles are all affected. This will complicate the extraction of the fundamental parameters, i.e. the number of extra dimensions $n$ and the

\footnotetext{
${ }^{4}$ Bulk graviton emission from non-rotating black holes is included in BLACKMAX.
} 
Planck scale $M_{D}$. More sophisticated analyses than those formulated for the non-rotating case are required and are currently under study.

Clearly if the LHC enters the energy region where extra dimensions can be probed and microblack-holes can be produced, the data will give us insights on the energy scales involved. Any future colliders will then no doubt be around or above the energy threshold for these phenomena, expected to be well over the $\mathrm{TeV}$ range. It would be useful to be able to span a range up to five to ten times the fundamental Planck scale, to study quantum gravity effects and reach a region where the black hole dynamics is expected to be described by general relativity. While multi-TeV lepton colliders can perhaps offer precision measurements in the domain just beyond the Planck scale, an energy upgrade of the LHC (DLHC) or even a Very Large Hadron collider may be required for a complete mapping of this new regime. In some cases it is also possible that the cross sections of the black hole production will be low (eg if apparent horizon effects are important) so that the LHC will only see some evidence of black hole production but does not allow for a detailed study. Then the LHC luminosity upgrade may be essential.

\subsection{Flavour Physics}

In the last few years there has been great experimental progress in quark and lepton flavour physics. In the quark sector, the validity of the Standard Model has been strongly reinforced by a series of challenging tests $[295,296]$. All the SM parameters controlling quark-flavour dynamics (quark masses and CKM angles) have been determined with good accuracy. More important, the measurements of several suppressed observables, such as $\Delta M_{B_{d}}, \Delta M_{B_{s}}, \mathcal{A}^{\mathrm{CP}}(B \rightarrow K \Psi)$, $\mathcal{B}\left(B \rightarrow X_{s} \gamma\right), \epsilon_{K}$, do not show significant deviations from the $\mathrm{SM}$. The situation is somehow similar to the flavour-conserving electroweak precision observables (EWPO) after LEP: the SM works very well and genuine one-loop electroweak effects have been tested with relative accuracy in the 10\%-30\% range. Similarly to the EWPO case, also in the quark flavour sector New Physics (NP) effects can only appear as a small correction to the leading SM contribution.

If NP respects the SM gauge symmetry, as we expect from general arguments, the corrections to low-energy flavour-violating amplitudes in the quark sector can be written in the following general form

$$
\mathcal{A}\left(q_{i} \rightarrow q_{j}+X\right)=\mathcal{A}_{0}\left[\frac{c_{\mathrm{SM}}}{M_{W}^{2}}+\frac{c_{\mathrm{NP}}}{\Lambda^{2}}\right],
$$

where $\Lambda$ is the energy scale of the new degrees of freedom. This structure is very general: the coefficients $c_{\mathrm{SM}(\mathrm{NP})}$ may include appropriate CKM factors and eventually a $\sim 1 /\left(16 \pi^{2}\right)$ suppression if the amplitude is loop-mediated. Given our ignorance about $c_{\mathrm{NP}}$, the values of the scale $\Lambda$ probed by present experiments vary over a wide range. However, the general result in Eq. (4.6) allow us to

predict how these bounds will improve with future experiments: the sensitivity on $\Lambda$ scale as $N^{1 / 4}$, where $N$ is the number of events used to measure the observable. This implies that is not easy to increase substantially the energy reach with indirect NP searches only.

On the other hand, if $\Lambda$ is just above the electroweak scale (and the LHC will soon provide a clear evidence of some new states), then we are already learning a lot about the couplings of these new degrees of freedom from flavour observables. Indeed to keep $\Lambda$ close to the electroweak scale we need some alignment between the SM and NP flavour structures. Natural possibilities 

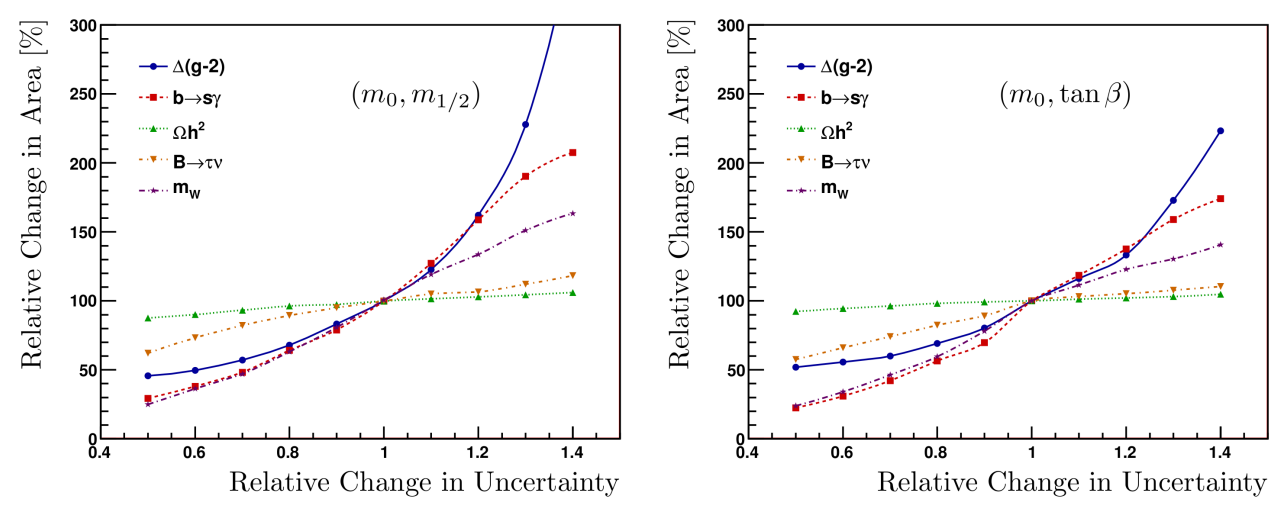

Fig. 4.11: Relative sizes of the $95 \%$ C.L. areas in the $\left(m_{0}, m_{1 / 2}\right)$ plane (left) and in the $\left(m_{0}, \tan \beta\right)$ plane (right) as a function of the hypothetical errors of various low-energy observables (plus $M_{W}$ and $\Omega_{\mathrm{CDM}} h^{2}$ ) [193]. The error scaling is relative to the current combined theory and experimental error.

are the Minimal Flavour Violation (MFV) hypothesis [297], which could easily be implemented in supersymmetric models or the so-called RS-GIM protection, which is at work in models with warped extra dimensions [298]. In all these cases improving the existing constraints on low-energy flavour-violating observables is necessary to improve our knowledge about some of the fundamental couplings of the NP model.

An example of the interest of improving measurements in the quark flavour sector, even in a framework where the impact of flavour observables is minimal, such as the constrained MSSM, is shown in Fig. 4.11. Here we illustrate the present impact of various low-energy measurements in constraining the parameter-space of the model, and the possible future impact assuming a reduction of the present errors [193]. Even if the flavour structure of the CMSSM is completely specified, helicity-suppressed observables such as $\mathcal{B}(B \rightarrow \tau \nu), \mathcal{B}\left(B \rightarrow X_{s} \gamma\right)$, and $\mathcal{B}\left(B_{s} \rightarrow \mu^{+} \mu^{-}\right.$) (not explicitly shown in Fig. 4.11 because of the present weak bound), are very useful in constraining the model because of their large sensitivity to $\tan \beta=v_{d} / v_{u}$. Since $\tan \beta$ cannot be determined with high accuracy from high- $p_{t}$ physics, these low-energy observables will remain key measurements also in the LHC era.

While helicity suppressed $B$-physics observables are very interesting in the CMSSM, $K$ and $D$ decays are more interesting in different NP frameworks. Altogether the set of low-energy observables to be measured with higher precision in the quark sector is quite limited. In several cases we are already dominated by irreducible theoretical uncertainties: the theoretical error on $c_{\mathrm{SM}}$ in Eq. (4.6) prevents the observation of possible NP effects. However, there are a few windows for very interesting dedicated new experiments. A notable example are the ultra-rare $K \rightarrow \pi \nu \bar{\nu}$ decays. Here the irreducible theoretical errors are very small [299] and these decays modes are quite interesting in the MSSM with non-MFV sources of flavour symmetry breaking [300], and in various models with extra dimensions [301].

Compared to the quark sector, the situation of flavour physics in the lepton sector is more uncertain but also more exciting. The discovery of neutrino oscillations has clearly revealed new flavour structures beside the three SM Yukawa couplings. We have not yet enough information to unambiguously determine how the SM Lagrangian should be modified in order to describe the 
phenomenon of neutrino oscillations. However, natural explanations point toward the existence of new degrees of freedom with explicit breaking of lepton number at very high energy scales, in agreement with the expectations of Grand Unified Theories (GUT). In several realistic supersymmetric frameworks, the new sources of lepton-flavour violation (LFV) should give rise to visible effects also in the charged-lepton sector. For this reason improved searches of LFV processes such as $\mu \rightarrow e \gamma, \tau \rightarrow \mu \gamma$, or $\mu \rightarrow e$ conversion in nuclei, are particularly interesting. A significant step forward in this field is expected soon by the MEG experiment, which should reach a sensitivity on $\mathcal{B}(\mu \rightarrow e \gamma)$ around $10^{-13}$, two orders of magnitude below the present one, covering a significant parameter region of realistic supersymmetric models [302]. However, we stress that also for LFV processes the general decomposition in Eq. (4.6) is valid: in this case $c_{\mathrm{SM}}=0$, hence we are never limited by irreducible theoretical errors, but the mild sensitivity on $\Lambda$ implied by the $N^{1 / 4}$ scaling is still valid.

\subsection{Summary and conclusions of WG4}

A broad spectrum of signatures for new phenomena have been extensively studied for the LHC. Many of the new physics signals discussed here are accessible with low luminosity at the LHC, extending the search reach of the Tevatron. In some cases, e.g., the 4 generation SM, early operations at the LHC can either discover or exclude the model. In other cases, the increasing luminosity (and energy) of the LHC with time will extend the reach and/or will allow for measurements of the properties of the new states. Some scenarios, such as the Little Higgs Model, may require several tens of $\mathrm{fb}^{-1}$ of data. For $Z^{\prime}$-like objects, as discussed in detail in this report, discovery depends on the mass and the couplings: early observation is possible but in other cases $100 \mathrm{fb}^{-1}$ at $14 \mathrm{TeV}$ may be barely enough to claim evidence. In addition, new physics could well be observed with unusual signatures. The LHC experiments have prepared for that to the best of their abilities and a signal could be observed early on.

Once a discovery has been made at the LHC, it will be imperative to determine the underlying theory which gives rise to the new phenomena. This has been the hallmark of the physics case for a high energy $e^{+} e^{-}$linear collider [40,124,303]. The sLHC luminosity upgrade has the capability to add crucial information on the properties of new physics (in addition to increasing the search sensitivity), although full studies have yet to be performed. A linear collider, with its clean environment, known initial state, and polarized beams, is unparalleled in terms of its abilities to conduct ultra-precise measurements of new (as well as SM) phenomena, as long as the new physics scale is within reach of the machine. The physics case has yet to be established for the LHeC and the muon collider. In the former case, it is clear from HERA data that much ground can be covered. In the latter case, a background saturated environment, challenging vertex measurements and lack of polarized beams, as well as a significant loss of forward coverage due to shielding will make precision measurements challenging.

A roadmap of sensitivities of various colliders for new physics scenarios is a useful guide to determine a machine's ability to probe new interactions. To that end, the sensitivities of various future facilities for discovering some scenarios are reproduced in Table 4.1 (from [304]). While lepton colliders allow for much more precise and complete measurements, as stated above, their effectiveness depends on the scale of the new physics. However, for the scenarios listed in this Table, the ILC sensitivity essentially matches that of the LHC, while CLIC matches that of the 


\begin{tabular}{|c|c|c|c|c|c|c|}
\hline Process & $\begin{array}{c}\text { LHC } \\
14 \mathrm{TeV} \\
100 \mathrm{fb}^{-1}\end{array}$ & $\begin{array}{c}\text { sLHC } \\
14 \mathrm{TeV} \\
1000 \mathrm{fb}^{-1}\end{array}$ & $\begin{array}{c}\text { DLHC } \\
28 \mathrm{TeV} \\
100 \mathrm{fb}^{-1}\end{array}$ & $\begin{array}{c}\text { VLHC } \\
200 \mathrm{TeV} \\
100 \mathrm{fb}^{-1}\end{array}$ & $\begin{array}{c}\text { ILC } \\
0.8 \mathrm{TeV} \\
500 \mathrm{fb}^{-1}\end{array}$ & $\begin{array}{c}\text { CLIC } \\
5 \mathrm{TeV} \\
1000 \mathrm{fb}^{-1}\end{array}$ \\
\hline$Z^{\prime}$ & 5 & 6 & 8 & 35 & 8 & 30 \\
\hline $\mathrm{ED}(\delta=2)$ & 9 & 12 & 15 & 65 & $5-8.5$ & $30-55$ \\
\hline excited quarks & 6.5 & 7.5 & 9.5 & 75 & 0.8 & 5 \\
\hline$\Lambda_{\text {compositness }}$ & 30 & 40 & 40 & 100 & 100 & 400 \\
\hline
\end{tabular}

Table 4.1: Illustrative reach in $\mathrm{TeV}$ for the different new physics scenarios. The $\mathrm{Z}$ ' and EDs for the LCs are indirect reach limits from precision measurements.

VLHC. Equally important is to understand the consequences if NO new signal is observed at the LHC, and how to tackle that scenario with future machines, although all evidence points to new physics at the Terascale.

In conclusion, we look forward to exciting times ahead with spectacular discoveries at the LHC, and to these discoveries pointing the way forward to the next machine. 


\section{Appendix}

\section{Future Colliders Overview Series}

The primary theme of the Theory Institute was to organise our thoughts around four broadlybased physics signature categories, and then to ask how various colliders can uncover and study the corresponding physics theories. The talks, discussions and write-ups of the four Working Groups are the products of that effort. Special attention was given to the near-term capabilities of the LHC, as the start-up of that collider is currently approaching. Results from the Tevatron were also integrated in the studies, along with some anticipations of gains one might obtain from the projected LHC luminosity upgrade (sLHC), an LHC-electron collider (LHeC), a future highenergy $e^{+} e^{-}$collider (ILC and/or CLIC), and a muon collider.

In addition to this effort placed within the Working Group structure, we also commissioned presentations that were focused exclusively on future technologies. We wanted summaries of the physics case for each possible future facility and a summary of its technology status. We were fortunate to have ten leaders of their respective colliders agree to participate in this "Future Colliders Overview Series."

Aurelio Juste's presentation was on the latest developments at the Tevatron and the expectations for Tevatron machine running, studying the Standard Model, and discovering new physics in the next few years. In particular, slides 48-55 have a discussion of current Higgs boson limits and future search prospects at the Tevatron. Lyn Evans gave a comprehensive talk on the LHC accelerator status, with comments about the path to upgrading to a higher-luminosity machine (the sLHC). Michelangelo Mangano gave the physics case for the sLHC, and summarised some of the attending challenges as well. Max Klein and Emmanuelle Perez spoke about the technology challenges and physics opportunities for turning the LHC machine into an electron-proton collider (the $\mathrm{LHeC}$ ).

We had four talks devoted to future $e^{+} e^{-}$colliders. Brian Foster and Klaus Desch discussed the technology and physics case for the ILC, which is a mature design for sub- $\mathrm{TeV} e^{+} e^{-}$collisions with potential for expanding to larger energies depending on site and design specifics. Jean-Pierre Delahaye and Marco Battaglia gave parallel talks for CLIC, which is in its R\&D phase, with the goal of providing a design option that enables colliding $e^{+} e^{-}$beams at energies of several $\mathrm{TeV}$ in the center of mass.

Finally, the status of the muon collider was discussed by Robert Palmer, who explained the advantages and limitations of pursuing muon collider technologies. The "easy parts" and the "hard parts" of reaching a final design for a collider were carefully detailed. An R\&D programme was proposed that would be necessary to determine the feasibility of a muon collider.

Below we list all ten talks in the series in alphabetical order of the speakers names. The slides for each talk can be accessed directly by the URLs given. In addition to the full URL, we have provided for each talk a shortened URL in parentheses from the http://is.gd/ service for the reader's convenience. 


\section{Talks delivered in the Future Colliders Overview Series}

Marco Battaglia, Physics case for CLIC, 19 Feb 2009 (is.gd/3gdkx)

http://indico. cern. ch/contributionDisplay . py? contribId=11\&conf $I d=40437$

Jean-Pierre Delahaye, Technology path to CLIC, 19 Feb 2009 (is.gd/3gdsG)

http://indico . cern. ch/contributionDisplay . py? contribId $=13 \&$ conf $I d=40437$

Klaus Desch, Physics case for the ILC, 17 Feb 2009 (is.gd/3geif)

http://indico. cern. ch/contributionDisplay $\cdot$ py? contribId=1\&conf Id=40437

Lyn Evans, LHC accelerator status and upgrade plans, 10 Feb 2009 (is.gd/3gelI)

http://indico. cern. ch/contributionDisplay. py? contribId=0\&conf $I d=40437$

Brian Foster, Technology progress report of the ILC, 17 Feb 2009 (is.gd/3genR) http: //indico. cern. ch/contributionDisplay $\cdot$ py? contribId=3\&conf $I d=40437$

Aurelio Juste, Recent results and prospects from the Tevatron, 26 Feb 2009 (is.gd/3gcij) http://indico. cern. ch/contributionDisplay . py? contribId=19\&conf $\mathrm{Id}=40437$

Max Klein, Towards a LHeC at the LHC, 18 Feb 2009 (is.gd/3gerX)

http://indico. cern. ch/contributionDisplay . py? contribId=9\&conf $I d=40437$

Michelangelo Mangano, Physics opportunities with the sLHC, 20 Feb 2009 (is.gd/3gevW) http://indico. cern. ch/contributionDisplay . py? contribId=5\&conf $I d=40437$

Robert Palmer, Muon collider technology status, 24 Feb 2009 (is.gd/3gey0) http://indico. cern. ch/contributionDisplay. py? contribId=14\&conf Id=40437

Emmanuelle Perez, Physics opportunities with the LHeC, 18 Feb 2009 (is.gd/3geAB) http://indico. cern. ch/contributionDisplay . py? contribId=7\&conf $\operatorname{Id}=40437$ 


\section{References}

[1] Tevatron New Phenomena and Higgs Working group [CDF Collaboration, D0 Collaboration], arXiv:0903.4001 [hep-ex].

[2] G. Aad et al. [The ATLAS Collaboration], "Expected Performance of the ATLAS Experiment - Detector, Trigger and Physics," arXiv:0901.0512.

[3] G. L. Bayatian et al. [CMS Collaboration], "CMS technical design report, volume II: Physics performance,” J. Phys. G 34 (2007) 995.

[4] A. Bredenstein, A. Denner, S. Dittmaier and S. Pozzorini, JHEP 0808 (2008) 108 [arXiv:0807.1248 [hep-ph]].

[5] A. Bredenstein, A. Denner, S. Dittmaier and S. Pozzorini, arXiv:0905.0110 [hep-ph].

[6] twiki.cern.ch/twiki/bin/view/Atlas/AtlasResultsEcmDependence .

[7] twiki.cern.ch/twiki/bin/view/CMS/

HiggsAnalysisSummary2009FebCombinedLimits .

[8] K. Assamagan, talk given at the $L H C 2 F C$ workshop, CERN, February 2009.

[9] [CDF Collaboration and D0 Collaboration], arXiv:0903.4001 [hep-ex].

[10] G. Bernardi, talk given at LeptonPhoton 2009, see: http://indico.desy.de/contributionDisplay.py?contribId=15\&conf $\mathrm{Id}=1761$.

[11] R. Barate et al. [LEP Working Group for Higgs boson searches and ALEPH Collaboration and DELPHI Collaboration and L3 Collaboration and OPAL Collaboration], Phys. Lett. B 565 (2003) 61 [arXiv:hep-ex/0306033].

[12] S. Schael et al. [ALEPH Collaboration and DELPHI Collaboration and L3 Collaboration and OPAL Collaboration], Eur. Phys. J. C 47 (2006) 547 [arXiv:hep-ex/0602042].

[13] M. Dührssen, S. Heinemeyer, H. Logan, D. Rainwater, G. Weiglein and D. Zeppenfeld, Phys. Rev. D 70 (2004) 113009 [arXiv:hep-ph/0406323].

[14] J. M. Butterworth, A. R. Davison, M. Rubin and G. P. Salam, Phys. Rev. Lett. 100, 242001 (2008) [arXiv:0802.2470 [hep-ph]].

[15] E. Gabrielli, F. Maltoni, B. Mele, M. Moretti, F. Piccinini and R. Pittau, Nucl. Phys. B 781 (2007) 64 [arXiv:hep-ph/0702119].

[16] D. L. Rainwater, Phys. Lett. B 503 (2001) 320 [arXiv:hep-ph/0004119].

[17] A. Ballestrero, G. Bevilacqua and E. Maina, JHEP 0808 (2008) 059 [arXiv:0806.4075 [hep$\mathrm{ph}]]$.

[18] R. Lafaye, T. Plehn, M. Rauch, D. Zerwas and M. Dührssen, arXiv:0904.3866 [hep-ph].

[19] M. Dührssen, ATL-PHYS-2003-030, available from cdsweb . cern . ch .

[20] C. Ruwiedel, N. Wermes and M. Schumacher, Eur. Phys. J. C 51 (2007) 385.

[21] V. Hankele, G. Klamke, D. Zeppenfeld and T. Figy, Phys. Rev. D 74 (2006) 095001 [arXiv:hep-ph/0609075].

[22] T. Plehn, D. L. Rainwater and D. Zeppenfeld, Phys. Rev. Lett. 88 (2002) 051801 [arXiv:hep$\mathrm{ph} / 0105325]$.

[23] F. Gianotti et al., Eur. Phys. J. C 39, 293 (2005) [arXiv:hep-ph/0204087].

[24] G. Aarons et al. [ILC Collaboration], arXiv:0709.1893 [hep-ph].

[25] H. Braun et al. [CLIC Study Team Collaboration], “CLIC 2008 parameters”, CERN-OPEN- 
2008-021, CLIC-Note-764.

[26] W. Scandale and F. Zimmermann, Nucl. Phys. Proc. Suppl. 177-178 (2008) 207.

[27] G. Ambrosio et al. [VLHC Design Study Group], "Design study for a staged very large hadron collider,"

see: tdserver1.fnal.gov/tddoc/DesignStudyReport/upload/PDF/ .

[28] indico . cern. ch/conferenceDisplay . py? conf Id=20082 .

[29] care-hhh.web.cern.ch/CARE-HHH .

[30] B. Autin, A. Blondel and J. R. . Ellis et al., "Prospective study of muon storage rings at CERN" CERN-99-02.

[31] R. Raja et al. [Neutrino Factory and Muon Collider Collaboration], arXiv:hep-ex/0108041.

[32] C. Blochinger et al., arXiv:hep-ph/0202199.

[33] U. Baur, T. Plehn and D. L. Rainwater, Phys. Rev. D 69 (2004) 053004 [arXiv:hep$\mathrm{ph} / 0310056]$.

[34] F. Mazzucato, A. Blondel and A. Clark, ATL-PHYS-2002-029, available from cdsweb.cern.ch .

[35] S. Y. Choi, D. J. Miller, M. M. Muhlleitner and P. M. Zerwas, Phys. Lett. B 553 (2003) 61 [arXiv:hep-ph/0210077].

[36] R. M. Godbole, D. J. 2. Miller and M. M. Muhlleitner, JHEP 0712 (2007) 031 [arXiv:0708.0458 [hep-ph]].

[37] P. S. Bhupal Dev, A. Djouadi, R. M. Godbole, M. M. Muhlleitner and S. D. Rindani, Phys. Rev. Lett. 100 (2008) 051801 [arXiv:0707.2878 [hep-ph]].

[38] C. P. Buszello, I. Fleck, P. Marquard and J. J. van der Bij, Eur. Phys. J. C 32 (2004) 209 [arXiv:hep-ph/0212396].

[39] K. Jakobs, Eur. Phys. J. C 59 (2009) 463.

[40] J. A. Aguilar-Saavedra et al. [ECFA/DESY LC Physics Working Group], arXiv:hep$\mathrm{ph} / 0106315$, see tesla.desy.de/new_pages/TDR_CD/start.html .

[41] J. Brau et al., "International Linear Collider reference design report. 1: Executive summary. 2: Physics at the ILC. 3: Accelerator. 4: Detectors".

[42] S. Heinemeyer et al., arXiv:hep-ph/0511332.

[43] T. Behnke et al. "Intended ILD Letter of Intent", see: wWw .ilcild.org/documents/ild-letter-of-intent/LOI.pdf/view .

[44] K. Ackermann et al., "Extended joint ECFA/DESY study on physics and detector for a linear e+ e- collider. Proceedings, Summer Colloquium, Amsterdam, Netherlands, April 4, 2003."

[45] A. Gay, Eur. Phys. J. C 49 (2007) 489 [arXiv:hep-ph/0604034].

[46] A. Imhof, LC-PHSM-2006-001 (2006), see: www . desy . de/ lcnotes/notes .html .

[47] J. Erler, S. Heinemeyer, W. Hollik, G. Weiglein and P. M. Zerwas, Phys. Lett. B 486 (2000) 125 [arXiv:hep-ph/0005024].

[48] H. Flacher, M. Goebel, J. Haller, A. Hocker, K. Moenig and J. Stelzer, arXiv:0811.0009 [hep-ph].

[49] N. T. Meyer and K. Desch, Eur. Phys. J. C 35 (2004) 171. 
[50] M. Battaglia, arXiv:hep-ph/0211461.

[51] R. Hawkings and K. Monig, Eur. Phys. J. direct C 1 (1999) 8 [arXiv:hep-ex/9910022].

[52] G. Wilson, LC-PHSM-2001-009, see: www . desy.de/ lcnotes/notes . html .

[53] O. Buchmueller et al., Phys. Lett. B 657 (2007) 87 [arXiv:0707.3447 [hep-ph]].

[54] B. Badelek et al. [ECFA/DESY Photon Collider Working Group], Int. J. Mod. Phys. A 19 (2004) 5097 [arXiv:hep-ex/0108012].

[55] E. Accomando et al. [CLIC Physics Working Group], arXiv:hep-ph/0412251.

[56] M. Battaglia, E. Boos and W. M. Yao, arXiv:hep-ph/0111276.

[57] M. Battaglia, J. Phys. G 35 (2008) 095005 [arXiv:0807.1299 [hep-ex]].

[58] M. Goebel, talk given at Rencontres de Moriond EW 2009.

[59] lepewwg.web.cern. ch/LEPEWWG/Wel come.html .

[60] ATLAS Collaboration, Detector and Physics Performance Technical Design Report, CERN/LHCC/99-15 (1999), see: atlasinfo . cern. ch/Atlas/GROUPS/PHYSICS/TDR/access . html .

[61] V. Barger, P. Langacker, M. McCaskey, M. J. Ramsey-Musolf and G. Shaughnessy, Phys. Rev. D 77, 035005 (2008) [arXiv:0706.4311 [hep-ph]].

[62] S. Dawson and W. Yan, arXiv:0904.2005 [hep-ph].

[63] G. Cacciapaglia, A. Deandrea and J. Llodra-Perez, arXiv:0901.0927 [hep-ph]

[64] I. F. Ginzburg, M. Krawczyk and P. Osland, arXiv:hep-ph/0101208.

[65] M. Schram, talk given at the $L H C 2 F C$ workshop, CERN, February 2009.

[66] G. F. Giudice, C. Grojean, A. Pomarol and R. Rattazzi, JHEP 0706 (2007) 045 [arXiv:hep$\mathrm{ph} / 0703164]$.

[67] N. Amapane et al. CMS Note 2007/005, available from cdsweb . cern . ch .

[68] P. Govoni [CMS Collaboration], AIP Conf. Proc. 1078 (2009) 229.

[69] M. S. Carena, J. R. Ellis, A. Pilaftsis and C. E. M. Wagner, Phys. Lett. B 495 (2000) 155 [arXiv:hep-ph/0009212].

[70] V. Buescher and K. Jakobs, Int. J. Mod. Phys. A 20 (2005) 2523 [arXiv:hep-ph/0504099].

[71] M. Schumacher, Czech. J. Phys. 54 (2004) A103; arXiv:hep-ph/0410112.

[72] E. Accomando et al., arXiv:hep-ph/0608079.

[73] R. Dermisek and J. F. Gunion, Phys. Rev. Lett. 95, 041801 (2005) [arXiv:hep-ph/0502105].

[74] R. Dermisek and J. F. Gunion, Phys. Rev. D 73, 111701 (2006) [arXiv:hep-ph/0510322].

[75] A. Djouadi, Phys. Lett. B 435 (1998) 101 [arXiv:hep-ph/9806315].

[76] A.V. Manohar and M.B. Wise, Phys. Lett. B 636 (2006) 107 [arXiv:hep-ph/0601212].

[77] G. F. Giudice, R. Rattazzi and J. D. Wells, Nucl. Phys. B 595 (2001) 250 [arXiv:hep$\mathrm{ph} / 0002178]$.

[78] T. Binoth and J. J. van der Bij, Z. Phys. C 75 (1997) 17 [arXiv:hep-ph/9608245].

[79] J. R. Espinosa and J. F. Gunion, Phys. Rev. Lett. 82, 1084 (1999) [arXiv:hep-ph/9807275].

[80] S. Chang, R. Dermisek, J. F. Gunion and N. Weiner, Ann. Rev. Nucl. Part. Sci. 58 (2008) 75 [arXiv:0801.4554 [hep-ph]].

[81] P. Bechtle, O. Brein, S. Heinemeyer, G. Weiglein and K. E. Williams, arXiv:0811.4169 [hep-ph]. 
[82] K. E. Williams and G. Weiglein, Phys. Lett. B 660 (2008) 217 [arXiv:0710.5320 [hep-ph]].

[83] D. K. Ghosh, R. M. Godbole and D. P. Roy, Phys. Lett. B 628 (2005) 131 [arXiv:hep$\mathrm{ph} / 0412193]$.

[84] P. Bandyopadhyay, A. Datta, A. Datta and B. Mukhopadhyaya, Phys. Rev. D 78 (2008) 015017 [arXiv:0710.3016 [hep-ph]].

[85] J. R. Forshaw, J. F. Gunion, L. Hodgkinson, A. Papaefstathiou and A. D. Pilkington, JHEP 0804, 090 (2008) [arXiv:0712.3510 [hep-ph]].

[86] R. Dermisek and J. F. Gunion, Phys. Rev. D 79, 055014 (2009) [arXiv:0811.3537 [hep-ph]].

[87] V. D. Barger, M. S. Berger, J. F. Gunion and T. Han, Phys. Rept. 286, 1 (1997) [arXiv:hep$\mathrm{ph} / 9602415]$.

[88] O. J. P. Eboli and D. Zeppenfeld, Phys. Lett. B 495 (2000) 147 [arXiv:hep-ph/0009158].

[89] M. Schumacher, "Investigation of invisible decays of the Higgs boson at a future $e^{+} e^{-}$linear collider,” LC-PHSM-2003-096.

[90] H. Davoudiasl, T. Han and H.E. Logan, Phys. Rev. D 71, 115007 (2005) [arXiv:hep$\mathrm{ph} / 0412269]$.

[91] W. Lohmann, M. Ohlerich, A. Raspereza and A. Schalicke, [arXiv:0710.2602 [hep-ex]].

[92] K. Belotsky, V. A. Khoze, A. D. Martin and M. G. Ryskin, Eur. Phys. J. C 36, 503 (2004) [arXiv:hep-ph/0406037].

[93] F. Sannino and K. Tuominen, Phys. Rev. D 71 (2005) 051901 [arXiv:hep-ph/0405209].

[94] D. D. Dietrich and F. Sannino, Phys. Rev. D 75 (2007) 085018 [arXiv:hep-ph/0611341].

[95] A. Belyaev, R. Foadi, M. T. Frandsen, M. Jarvinen, F. Sannino and A. Pukhov, Phys. Rev. D 79 (2009) 035006 [arXiv:0809.0793 [hep-ph]].

[96] T. Appelquist and F. Sannino, Phys. Rev. D 59, 067702 (1999) [arXiv:hep-ph/9806409].

[97] N. D. Christensen and R. Shrock, Phys. Lett. B 632, 92 (2006) [arXiv:hep-ph/0509109].

[98] G. H. Brooijmans et al., arXiv:0802.3715 [hep-ph].

[99] E. Eichten, K. D. Lane and J. Womersley, Phys. Lett. B 405 (1997) 305 [arXiv:hep$\mathrm{ph} / 9704455]$.

[100] K. Lane and A. Martin, arXiv:0907.3737 [hep-ph].

[101] M. E. Peskin and T. Takeuchi, Phys. Rev. Lett. 65 (1990) 964.

[102] R. Barbieri, G. Isidori, V. S. Rychkov and E. Trincherini, Phys. Rev. D 78 (2008) 036012 [arXiv:0806.1624 [hep-ph]].

[103] R. Foadi and F. Sannino, Phys. Rev. D 78 (2008) 037701 [arXiv:0801.0663 [hep-ph]].

[104] R. Foadi, M. Jarvinen and F. Sannino, Phys. Rev. D 79 (2009) 035010 [arXiv:0811.3719 [hep-ph]].

[105] A. Belyaev, arXiv:0711.1919 [hep-ph].

[106] O. Cata, G. Isidori and J. F. Kamenik, arXiv:0905.0490 [hep-ph].

[107] T. Ohl and C. Speckner, Phys. Rev. D 78 (2008) 095008 [arXiv:0809.0023 [hep-ph]].

[108] G. Cacciapaglia, A. Deandrea and S. De Curtis, arXiv:0906.3417 [hep-ph].

[109] J. Hirn, A. Martin and V. Sanz, JHEP 0805, 084 (2008) [arXiv:0712.3783 [hep-ph]].

[110] J. Hirn, A. Martin and V. Sanz, Phys. Rev. D 78, 075026 (2008) [arXiv:0807.2465 [hep-ph]].

[111] K. Agashe, C. Csaki, C. Grojean and M. Reece, JHEP 0712, 003 (2007) [arXiv:0704.1821 
[hep-ph]].

[112] R. Casalbuoni, S. De Curtis, D. Dolce and D. Dominici, Phys. Rev. D 71 (2005) 075015 [arXiv:hep-ph/0502209].

[113] R. Casalbuoni, S. De Curtis, D. Dominici and R. Gatto, Phys. Lett. B 155 (1985) 95.

[114] E. Accomando, S. De Curtis, D. Dominici and L. Fedeli, arXiv:0807.5051 [hep-ph].

[115] E. Accomando, M. Battaglia, S. De Curtis, D. Dominici and L. Fedeli, in preparation.

[116] M. Battaglia, S. De Curtis and D. Dominici, JHEP 0212 (2002) 004 [arXiv:hep$\mathrm{ph} / 0210351]$.

[117] N. Amapane et al., CMS AN-2007/005.

[118] J. M. Butterworth, B. E. Cox and J. R. Forshaw, Phys. Rev. D 65 (2002) 096014 [arXiv:hep$\mathrm{ph} / 0201098]$.

[119] E. Accomando, A. Ballestrero, S. Bolognesi, E. Maina and C. Mariotti, JHEP 0603, 093 (2006) [arXiv:hep-ph/0512219].

[120] A. Ballestrero, A. Belhouari, G. Bevilacqua, V. Kashkan and E. Maina, Comput. Phys. Commun. 180, 401 (2009) [arXiv:0801.3359 [hep-ph]].

[121] A. Ballestrero, G. Bevilacqua and E. Maina, arXiv:0812.5084 [hep-ph].

[122] T. Appelquist and C. W. Bernard, Phys. Rev. D 22 (1980) 200.

[123] J. Bagger, S. Dawson and G. Valencia, Nucl. Phys. B 399 (1993) 364 [arXiv:hep$\mathrm{ph} / 9204211]$.

[124] G. Weiglein, et al, Phys. Rept. 426 (2006), 47 [arXiv:hep-ph/0410364]

[125] A. S. Belyaev, et al, Phys. Rev. D59 (1999) 015022 [arXiv:hep-ph/9805229]

[126] O. J. P. Eboli, M. C. Gonzalez-Garcia and J. K. Mizukoshi, Phys. Rev. D74, (2006) 073005 [arXiv:hep-ph/0606118]

[127] A. Dobado, M. J. Herrero, J. R. Pelaez and E. Ruiz Morales, Phys. Rev. D62 (2000) 055011 [arXiv:hep-ph/9912224]

[128] [CMS Collaboration], CMS Physics Analysis Summary JME-07-001 (2007).

[129] C. Cojocaru et al. [ATLAS Liquid Argon EMEC/HEC Collaboration], Nucl. Instrum. Meth. A 531 (2004) 481 [arXiv:physics/0407009].

[130] [CMS Collaboration], CMS Physics Analysis Summary JME-09-010 (2009), see cms-physics. web. cern.ch/cms-physics/public/JME-09-010-pas .pdf .

[131] [CMS Collaboration], CMS Physics Analysis Summary PFT-09-001 (2009), see cms-physics. web.cern.ch/cms-physics/public/PFT-09-001-pas.pdf .

[132] R. Adolphi et al. [CMS Collaboration], JINST 0803 (2008) S08004 [JINST 3 (2008) S08004].

[133] F. E. Paige, arXiv:hep-ph/9609373.

[134] I. Hinchliffe, F. E. Paige, M. D. Shapiro, J. Soderqvist and W. Yao, Phys. Rev. D 55 (1997) 5520 [arXiv:hep-ph/9610544].

[135] H. Bachacou, I. Hinchliffe and F. E. Paige, Phys. Rev. D 62 (2000) 015009 [arXiv:hep$\mathrm{ph} / 9907518]$.

[136] K. T. Matchev, F. Moortgat, L. Pape and M. Park, arXiv:0906.2417 [hep-ph].

[137] M. M. Nojiri, G. Polesello and D. R. Tovey, arXiv:hep-ph/0312317. 
[138] K. Kawagoe, M. M. Nojiri and G. Polesello, Phys. Rev. D 71 (2005) 035008 [arXiv:hep$\mathrm{ph} / 0410160]$.

[139] H. C. Cheng, D. Engelhardt, J. F. Gunion, Z. Han and B. McElrath, Phys. Rev. Lett. 100 (2008) 252001 [arXiv:0802.4290 [hep-ph]].

[140] H. C. Cheng, J. F. Gunion, Z. Han and B. McElrath, Phys. Rev. D 80 (2009) 035020 [arXiv:0905.1344 [hep-ph]].

[141] H. C. Cheng, J. F. Gunion, Z. Han, G. Marandella and B. McElrath, JHEP 0712 (2007) 076 [arXiv:0707.0030 [hep-ph]].

[142] B. C. Allanach et al. [Beyond the Standard Model Working Group], arXiv:hep-ph/0402295.

[143] B. Webber, arXiv:0907.5307 [hep-ph].

[144] T. Aaltonen et al. [CDF Collaboration], Phys. Rev. D 77 (2008) 112001 [arXiv:0708.3642 [hep-ex]].

[145] C. G. Lester and D. J. Summers, Phys. Lett. B 463 (1999) 99 [arXiv:hep-ph/9906349].

[146] A. Barr, C. Lester and P. Stephens, J. Phys. G 29 (2003) 2343 [arXiv:hep-ph/0304226].

[147] [CDF Collaboration] "Simultaneous Template-Based Top Quark Mass Measurement in the Lepton+Jets and Dilepton Channels Using $3 \mathrm{fb}^{-1}$ of CDF Data" CDF note 9769 (2009).

[148] W. S. Cho, K. Choi, Y. G. Kim and C. B. Park, Phys. Rev. Lett. 100 (2008) 171801 [arXiv:0709.0288 [hep-ph]].

[149] B. Gripaios, JHEP 0802 (2008) 053 [arXiv:0709.2740 [hep-ph]].

[150] A. J. Barr, B. Gripaios and C. G. Lester, JHEP 0802 (2008) 014 [arXiv:0711.4008 [hep-ph]].

[151] W. S. Cho, K. Choi, Y. G. Kim and C. B. Park, JHEP 0802 (2008) 035 [arXiv:0711.4526 [hep-ph]].

[152] M. Serna, JHEP 0806 (2008) 004 [arXiv:0804.3344 [hep-ph]].

[153] M. Burns, K. Kong, K. T. Matchev and M. Park, JHEP 0903 (2009) 143 [arXiv:0810.5576 [hep-ph]].

[154] H. C. Cheng and Z. Han, JHEP 0812 (2008) 063 [arXiv:0810.5178 [hep-ph]].

[155] L. Randall and D. Tucker-Smith, Phys. Rev. Lett. 101 (2008) 221803 [arXiv:0806.1049 [hep-ph]].

[156] A. J. Barr and C. Gwenlan, arXiv:0907.2713 [hep-ph].

[157] C. Lester and A. Barr, JHEP 0712 (2007) 102 [arXiv:0708.1028 [hep-ph]].

[158] G. G. Ross and M. Serna, Phys. Lett. B 665 (2008) 212 [arXiv:0712.0943 [hep-ph]].

[159] A. J. Barr, G. G. Ross and M. Serna, Phys. Rev. D 78 (2008) 056006 [arXiv:0806.3224 [hep-ph]].

[160] A. J. Barr, B. Gripaios and C. G. Lester, JHEP 0907 (2009) 072 [arXiv:0902.4864 [hep-ph]].

[161] L. T. Wang and I. Yavin, Int. J. Mod. Phys. A 23 (2008) 4647 [arXiv:0802.2726 [hep-ph]].

[162] A. J. Barr, Phys. Lett. B 596 (2004) 205 [arXiv:hep-ph/0405052].

[163] T. Goto, K. Kawagoe and M. M. Nojiri, Phys. Rev. D 70 (2004) 075016 [Erratum-ibid. D 71 (2005) 059902] [arXiv:hep-ph/0406317].

[164] J. M. Smillie and B. R. Webber, JHEP 0510 (2005) 069 [arXiv:hep-ph/0507170].

[165] A. Datta, K. Kong and K. T. Matchev, Phys. Rev. D 72 (2005) 096006 [Erratum-ibid. D 72 (2005) 119901] [arXiv:hep-ph/0509246]. 
[166] C. Athanasiou, C. G. Lester, J. M. Smillie and B. R. Webber, JHEP 0608 (2006) 055 [arXiv:hep-ph/0605286].

[167] M. Burns, K. Kong, K. T. Matchev and M. Park, JHEP 0810 (2008) 081 [arXiv:0808.2472 [hep-ph]].

[168] O. Gedalia, S. J. Lee and G. Perez, Phys. Rev. D 80 (2009) 035012 [arXiv:0901.4438 [hep$\mathrm{ph}]]$.

[169] L. T. Wang and I. Yavin, JHEP 0704 (2007) 032 [arXiv:hep-ph/0605296].

[170] A. J. Barr, JHEP 0602(2006) 042 [arXiv:hep-ph/0511115].

[171] M. Battaglia, A. Datta, A. De Roeck, K. Kong and K. T. Matchev, JHEP 0507 (2005) 033 [arXiv:hep-ph/0502041].

[172] M. Battaglia, A. K. Datta, A. De Roeck, K. Kong and K. T. Matchev, arXiv:hep-ph/0507284.

[173] G. Bhattacharyya, P. Dey, A. Kundu and A. Raychaudhuri, Phys. Lett. B 628 (2005) 141 [arXiv:hep-ph/0502031].

[174] M. R. Buckley, S. Y. Choi, K. Mawatari and H. Murayama, Phys. Lett. B 672 (2009) 275 [arXiv:0811.3030 [hep-ph]].

[175] F. Boudjema and R. K. Singh, JHEP 0907 (2009) 028 [arXiv:0903.4705 [hep-ph]].

[176] A. Alves, O. Eboli and T. Plehn, Phys. Rev. D 74 (2006) 095010 [arXiv:hep-ph/0605067].

[177] M. Kramer, E. Popenda, M. Spira and P. M. Zerwas, arXiv:0902.3795 [hep-ph].

[178] C. Csaki, J. Heinonen and M. Perelstein, JHEP 0710 (2007) 107 [arXiv:0707.0014 [hep$\mathrm{ph}]]$.

[179] W. S. Cho, K. Choi, Y. G. Kim and C. B. Park, Phys. Rev. D 79 (2009) 031701 [arXiv:0810.4853 [hep-ph]].

[180] A. Datta, G. L. Kane and M. Toharia, arXiv:hep-ph/0510204.

[181] G. L. Kane, A. A. Petrov, J. Shao and L. T. Wang, arXiv:0805.1397 [hep-ph].

[182] G. Hallenbeck, M. Perelstein, C. Spethmann, J. Thom and J. Vaughan, arXiv:0812.3135 [hep-ph].

[183] M. Perelstein and A. Weiler, JHEP 0903 (2009) 141 [arXiv:0811.1024 [hep-ph]].

[184] M. Perelstein and C. Spethmann, arXiv:0710.4148 [hep-ph].

[185] R. M. Godbole, M. Guchait and D. P. Roy, Phys. Lett. B 618, 193 (2005) [arXiv:hep$\mathrm{ph} / 0411306]$.

[186] M. Guchait and D. P. Roy, Phys. Lett. B 541 (2002) 356 [arXiv:hep-ph/0205015].

[187] S. Y. Choi, K. Hagiwara, Y. G. Kim, K. Mawatari and P. M. Zerwas, Phys. Lett. B 648 (2007) 207 [arXiv:hep-ph/0612237].

[188] T. Nattermann, K. Desch, P. Wienemann and C. Zendler, JHEP 0904 (2009) 057 [arXiv:0903.0714 [hep-ph]].

[189] K. Rolbiecki, J. Tattersall and G. Moortgat-Pick, arXiv:0909.3196 [hep-ph].

[190] N. Arkani-Hamed, P. Schuster, N. Toro, J. Thaler, L. T. Wang, B. Knuteson and S. Mrenna, arXiv:hep-ph/0703088.

[191] R. Lafaye, T. Plehn and D. Zerwas, arXiv:hep-ph/0404282.

[192] P. Bechtle, K. Desch and P. Wienemann, Comput. Phys. Commun. 174 (2006) 47 [arXiv:hep-ph/0412012]. 
[193] O. Buchmueller et al., JHEP 0809 (2008) 117 [arXiv:0808.4128 [hep-ph]].

[194] O. Buchmueller et al., arXiv:0907.5568 [hep-ph].

[195] B. C. Allanach and C. G. Lester, Phys. Rev. D 73 (2006) 015013 [arXiv:hep-ph/0507283].

[196] B. C. Allanach, C. G. Lester and A. M. Weber, JHEP 0612 (2006) 065 [arXiv:hep$\mathrm{ph} / 0609295]$.

[197] L. Roszkowski, R. Ruiz de Austri and R. Trotta, JHEP 0707 (2007) 075 [arXiv:0705.2012 [hep-ph]].

[198] J. R. Ellis, S. Heinemeyer, K. A. Olive and G. Weiglein, JHEP 0502 (2005) 013 [arXiv:hep$\mathrm{ph} / 0411216]$.

[199] J. R. Ellis, S. Heinemeyer, K. A. Olive and G. Weiglein, JHEP 0605 (2006) 005 [arXiv:hep$\mathrm{ph} / 0602220]$.

[200] J. R. Ellis, S. Heinemeyer, K. A. Olive, A. M. Weber and G. Weiglein, JHEP 0708 (2007) 083 [arXiv:0706.0652 [hep-ph]].

[201] S. Heinemeyer, X. Miao, S. Su and G. Weiglein, JHEP 0808 (2008) 087 [arXiv:0805.2359 [hep-ph]].

[202] J. Dunkley et al. [WMAP Collaboration], Astrophys. J. Suppl. 180 (2009) 306 [arXiv:0803.0586 [astro-ph]].

[203] M. Drees and M. M. Nojiri, Phys. Rev. D 47 (1993) 376 [arXiv:hep-ph/9207234].

[204] G. Jungman, M. Kamionkowski and K. Griest, Phys. Rept. 267 (1996) 195 [arXiv:hep$\mathrm{ph} / 9506380]$.

[205] P. Gondolo, J. Edsjo, P. Ullio, L. Bergstrom, M. Schelke and E. A. Baltz, JCAP 0407 (2004) 008 [arXiv:astro-ph/0406204].

[206] G. Belanger, F. Boudjema, A. Pukhov and A. Semenov, Comput. Phys. Commun. 176 (2007) 367 [arXiv:hep-ph/0607059].

[207] H. Baer, C. Balazs and A. Belyaev, JHEP 0203 (2002) 042 [arXiv:hep-ph/0202076].

[208] M. M. Nojiri, G. Polesello and D. R. Tovey, JHEP 0603 (2006) 063 [arXiv:hep-ph/0512204].

[209] E. A. Baltz, M. Battaglia, M. E. Peskin and T. Wizansky, Phys. Rev. D 74 (2006) 103521 [arXiv:hep-ph/0602187].

[210] G. Moortgat-Pick, V. Morton-Thurtle and P. Wienemann, "Dark matter searches at future colliders" (2009).

[211] K. Desch, plenary talk given at the $L H C 2 F C$ workshop, CERN, February 2009.

[212] S. Heinemeyer, S. Kraml, W. Porod and G. Weiglein, JHEP 0309 (2003) 075 [arXiv:hep$\mathrm{ph} / 0306181]$.

[213] K. Moenig, plenary talk given at the $L H C 2 F C$ workshop, CERN, February 2009.

[214] G. A. Moortgat-Pick et al., Phys. Rept. 460 (2008) 131 [arXiv:hep-ph/0507011].

[215] G. A. Blair, A. Freitas, H. U. Martyn, G. Polesello, W. Porod and P. M. Zerwas, Acta Phys. Polon. B 36 (2005) 3445 [arXiv:hep-ph/0512084].

[216] P. Bechtle, K. Desch, M. Uhlenbrock and P. Wienemann, arXiv:0907.2589 [hep-ph].

[217] J. E. Augustin et al., "Linear Collider, Final International Technology Recommendation Panel report."

[218] J. Brau et al. [ILC Collaboration], arXiv:0712.1950 [physics.acc-ph]. 
[219] B. Foster, plenary talk given at the $L H C 2 F C$ workshop, CERN, February 2009.

[220] M. Battaglia, plenary talk given at the $L H C 2 F C$ workshop, CERN, February 2009.

[221] J.-P. Delahaye, plenary talk given at the $L H C 2 F C$ workshop, CERN, February 2009.

[222] S. Kraml, plenary talk given at the $L H C 2 F C$ workshop, CERN, February 2009.

[223] S. Kraml, arXiv:0710.5117 [hep-ph].

[224] S. Hesselbach, arXiv:0709.2679 [hep-ph].

[225] O. Kittel, J. Phys. Conf. Ser. 171 (2009) 012094 [arXiv:0904.3241 [hep-ph]].

[226] P. Langacker, G. Paz, L. T. Wang and I. Yavin, JHEP 0707 (2007) 055 [arXiv:hep$\mathrm{ph} / 0702068]$.

[227] J. Ellis, F. Moortgat, G. Moortgat-Pick, J. M. Smillie and J. Tattersall, Eur. Phys. J. C 60 (2009) 633 [arXiv:0809.1607 [hep-ph]].

[228] F. Deppisch and O. Kittel, arXiv:0905.3088 [hep-ph].

[229] G. Moortgat-Pick, K. Rolbiecki, J. Tattersall and P. Wienemann, arXiv:0908.2631 [hep-ph].

[230] M. M. Nojiri, Phys. Rev. D 51 (1995) 6281 [arXiv:hep-ph/9412374].

[231] P. Bechtle, M. Berggren, J. List, P. Schade and O. Stempel, arXiv:0908.0876 [hep-ex].

[232] D.P. Roy, plenary talk given at the $L H C 2 F C$ workshop, CERN, February 2009.

[233] E. Boos, H. U. Martyn, G. A. Moortgat-Pick, M. Sachwitz, A. Sherstnev and P. M. Zerwas, Eur. Phys. J. C 30 (2003) 395 [arXiv:hep-ph/0303110].

[234] K. Desch, A. Imhof, Z. Was and M. Worek, Phys. Lett. B 579 (2004) 157 [arXiv:hep$\mathrm{ph} / 0307331]$.

[235] K. Desch, Z. Was and M. Worek, Eur. Phys. J. C 29 (2003) 491 [arXiv:hep-ph/0302046].

[236] T. Suehara and J. List, arXiv:0906.5508 [hep-ex].

[237] S. Heinemeyer, W. Hollik, A. M. Weber and G. Weiglein, JHEP 0804 (2008) 039 [arXiv:0710.2972 [hep-ph]].

[238] K. Desch, J. Kalinowski, G. Moortgat-Pick, K. Rolbiecki and W. J. Stirling, JHEP 0612 (2006) 007 [arXiv:hep-ph/0607104].

[239] P. Langacker, arXiv:0801.1345 [hep-ph].

[240] T. G. Rizzo, arXiv:hep-ph/0610104.

[241] M. S. Carena, A. Daleo, B. A. Dobrescu and T. M. P. Tait, Phys. Rev. D 70 (2004) 093009 [arXiv:hep-ph/0408098].

[242] M. Dittmar, A. S. Nicollerat and A. Djouadi, Phys. Lett. B 583 (2004) (2004) 111 [arXiv:hep-ph/0307020].

[243] A. Leike, Phys. Rept. 317 (1999) 143 [arXiv:hep-ph/9805494].

[244] J. L. Hewett and T. G. Rizzo, Phys. Rept. 183 (1989) 193.

[245] M. Cvetic and S. Godfrey, arXiv:hep-ph/9504216.

[246] T. G. Rizzo, eConf C960625, NEW136 (1996) [arXiv:hep-ph/9612440].

[247] T. G. Rizzo, JHEP 0811, 039 (2008) [arXiv:0809.4659 [hep-ph]].

[248] For a recent study, see F. Petriello and S. Quackenbush, Phys. Rev. D 77, 115004 (2008) [arXiv:0801.4389 [hep-ph]].

[249] www.ep.ph.bham.ac.uk/exp/LHeC//index.html .

[250] T. G. Rizzo, Phys. Rev. D 77 (2008) 115016 [arXiv:0804.0081 [hep-ph]]. 
[251] T. G. Rizzo, arXiv:0904.2534 [hep-ph].

[252] T. G. Rizzo, Phys. Rev. D 55, 5483 (1997) [arXiv:hep-ph/9612304].

[253] T. G. Rizzo, JHEP 0705, 037 (2007) [arXiv:0704.0235 [hep-ph]].

[254] A. Juste, private communication. CDF public note, www-cdf .fnal.gov/physics/new/top/2008/tprop/Tprime2.8/public.html .

[255] A. A. Affolder et al. [CDF Collaboration], Phys. Rev. Lett. 84, 835 (2000) [arXiv:hepex/9909027].

[256] J. Alwall et al., Eur. Phys. J. C 49, 791 (2007) [arXiv:hep-ph/0607115];

[257] M. Bobrowski, A. Lenz, J. Riedl and J. Rohrwild, Phys.Rev. D79, 113006 (2009) arXiv:0902.4883 [hep-ph];

[258] M. S. Chanowitz, Phys. Rev. D 79, 113008 (2009) [arXiv:0904.3570 [hep-ph]].

[259] M. Bona et al. [UTfit Collaboration], arXiv:0803.0659 [hep-ph].

[260] G. D. Kribs, T. Plehn, M. Spannowsky and T. M. P. Tait, Phys. Rev. D 76, 075016 (2007) [arXiv:0706.3718 [hep-ph]].

[261] M. S. Chanowitz, M. A. Furman and I. Hinchliffe, Phys. Lett. B 78, 285 (1978); Nucl. Phys. B 153, 402 (1979).

[262] J. A. Casas, J. R. Espinosa and M. Quiros, Phys. Lett. B 342, 171 (1995) [arXiv:hep$\mathrm{ph} / 9409458]$.

[263] T. Hambye and K. Riesselmann, Phys. Rev. D 55, 7255 (1997) [arXiv:hep-ph/9610272].

[264] J. F. Gunion, D. W. McKay and H. Pois, Phys. Lett. B 334, 339 (1994) [arXiv:hep$\mathrm{ph} / 9406249]$.

[265] R. Fok and G. D. Kribs, Phys. Rev. D 78, 075023 (2008) [arXiv:0803.4207 [hep-ph]]; S. W. Ham, S. K. Oh and D. Son, Phys. Rev. D 71, 015001 (2005) [arXiv:hep-ph/0411012].

[266] V. E. Ozcan, S. Sultansoy and G. Unel, arXiv:0808.0285 [hep-ph].

[267] E. Arik, S. A. Cetin and S. Sultansoy, Balk. Phys. Lett. 15N4, 1 (2007) [arXiv:0708.0241 [hep-ph]].

[268] P. Minkowski, Phys. Lett. B 67 (1977) 421.

[269] T. Yanagida, in Proceedings of the Workshop on the Unified Theory and the Baryon Number in the Universe, eds. O. Sawada et al., (KEK Report 79-18, Tsukuba, 1979), p. 95.

[270] M. Gell-Mann, P. Ramond and R. Slansky, in Supergravity, eds. P. van Nieuwenhuizen et al., (North-Holland, 1979), p. 315.

[271] S.L. Glashow, in Quarks and Leptons, Cargèse, eds. M. Lévy et al., (Plenum, 1980), p. 707.

[272] R. N. Mohapatra and G. Senjanović, Phys. Rev. Lett. 44 (1980) 912.

[273] W. Y. Keung and G. Senjanović, Phys. Rev. Lett. 50 (1983) 1427.

[274] T. Han and B. Zhang, Phys. Rev. Lett. 97, 171804 (2006).

[275] For a comparison for different colliders, see e.g., F. del Aguila, J. A. Aguilar-Saavedra and R. Pittau, J. Phys. Conf. Ser. 53, 506 (2006) [arXiv:hep-ph/0606198].

[276] R. Franceschini, T. Hambye and A. Strumia, arXiv:0805.1613 [hep-ph].

[277] A. Atre, T. Han, S. Pascoli and B. Zhang, JHEP 0905 (2009) 030 [arXiv:0901.3589 [hep$\mathrm{ph}]]$.

[278] W. Konetschny and W. Kummer, Phys. Lett. B 70 (1977) 433. 
[279] T. P. Cheng and L. F. Li, Phys. Rev. D 22 (1980) 2860.

[280] G. Lazarides, Q. Shafi and C. Wetterich, Nucl. Phys. B 181 (1981) 287.

[281] J. Schechter and J. W. F. Valle, Phys. Rev. D 22 (1980) 2227.

[282] R. N. Mohapatra and G. Senjanović, Phys. Rev. D 23 (1981) 165.

[283] E. J. Chun, K. Y. Lee and S. C. Park, Phys. Lett. B 566 (2003) 142 [arXiv:hep-ph/0304069];

[284] P. Fileviez Perez, T. Han, G. y. Huang, T. Li and K. Wang, Phys. Rev. D 78 (2008) 015018 [arXiv:0805.3536 [hep-ph]].

[285] R. Foot, H. Lew, X. G. He and G. C. Joshi, Z. Phys. C 44 (1989) 441.

[286] A. Arhrib, B. Bajc, D. K. Ghosh, T. Han, G. Y. Huang, I. Puljak and G. Senjanovic, arXiv:0904.2390 [hep-ph].

[287] [CMS Collaboration], CMS Physics Analysis Summary PAS-EXO-09-001 (2009).

[288] [CMS Collaboration], CMS Physics Analysis Summary PAS-EXO-08-003 (2008).

[289] P. Kanti, Lect. Notes Phys. 769, 387 (2009) [arXiv:0802.2218 [hep-th]].

[290] M. Shibata, H. Okawa and T. Yamamoto, Phys. Rev. D 78, 101501 (2008) [arXiv:0810.4735 [gr-qc]].

[291] H. Yoshino and V. S. Rychkov, Phys. Rev. D 71, 104028 (2005) [Erratum-ibid. D 77, 089905 (2008)] [arXiv:hep-th/0503171].

[292] J. A. Frost, J. R. Gaunt, M. O. P. Sampaio, M. Casals, S. R. Dolan, M. A. Parker and B. R. Webber, arXiv:0904.0979 [hep-ph]; projects .hepforge.org/charybdis2/

[293] D. C. Dai, G. Starkman, D. Stojkovic, C. Issever, E. Rizvi and J. Tseng, Phys. Rev. D 77, 076007 (2008) [arXiv:0711.3012 [hep-ph]]; projects .hepforge.org/blackmax/

[294] D. C. Dai, C. Issever, E. Rizvi, G. Starkman, D. Stojkovic and J. Tseng, arXiv:0902.3577 [hep-ph].

[295] M. Bona et al. [UTfit Collaboration], JHEP 0803 (2008) 049 [arXiv:0707.0636 [hep-ph]], updated results and plots available at: www.utfit.org/.

[296] J. Charles et al. [CKMfitter Group], Eur. Phys. J. C 41, 1 (2005) [arXiv:hep-ph/0406184], updated results and plots available at: ckmfitter.in2p3.fr.

[297] G. D’Ambrosio, G. F. Giudice, G. Isidori and A. Strumia, Nucl. Phys. B 645, 155 (2002) [arXiv:hep-ph/0207036].

[298] K. Agashe, G. Perez and A. Soni, Phys. Rev. D 71 (2005) 016002 [arXiv:hep-ph/0408134].

[299] A. J. Buras, M. Gorbahn, U. Haisch and U. Nierste, Phys. Rev. Lett. 95 (2005) 261805 [arXiv:hep-ph/0508165]; G. Isidori, F. Mescia and C. Smith, Nucl. Phys. B 718 (2005) 319 [arXiv:hep-ph/0503107].

[300] G. Isidori, F. Mescia, P. Paradisi, C. Smith and S. Trine, JHEP 0608 (2006) 064 [arXiv:hep$\mathrm{ph} / 0604074]$.

[301] M. Blanke, A. J. Buras, B. Duling, K. Gemmler and S. Gori, JHEP 0903 (2009) 108 [arXiv:0812.3803 [hep-ph]].

[302] J. Hisano, M. Nagai, P. Paradisi and Y. Shimizu, arXiv:0904.2080 [hep-ph].

[303] T. Abe et al. [American Linear Collider Working Group], arXiv:hep-ex/0106057.

[304] A. De Roeck, J. R. Ellis and F. Gianotti, arXiv:hep-ex/0112004. 